\title{
Footpoints of the giant molecular loops in the Galactic center region ${ }^{\star}$
}

\author{
D. Riquelme ${ }^{1}$, M. A. Amo-Baladrón ${ }^{2}$, J. Martín-Pintado ${ }^{2}$, R. Mauersberger ${ }^{1}$, S. Martín ${ }^{3,4}$, M. Burton ${ }^{5,6}$, \\ M. Cunningham ${ }^{5}$, P. A. Jones ${ }^{5}$, K. M. Menten ${ }^{1}$, L. Bronfman ${ }^{7}$, and R. Güsten ${ }^{1}$ \\ ${ }^{1}$ Max-Planck-Institut für Radioastronomie, Auf dem Hügel 69, 53121 Bonn, Germany \\ e-mail: riquelme@mpifr-bonn.mpg.de \\ ${ }^{2}$ Centro de Astrobiología (CSIC/INTA), Ctra. de Torrejón a Ajalvir km 4, 28850 Torrejón de Ardoz, Madrid, Spain \\ ${ }^{3}$ European Southern Observatory, Alonso de Córdova 3107, Vitacura, Santiago, Chile \\ ${ }^{4}$ Joint ALMA Observatory, Alonso de Córdova 3107, Vitacura, Santiago, Chile \\ ${ }^{5}$ School of Physics, University of New South Wales, Sydney, NSW 2052, Australia \\ ${ }^{6}$ Armagh Observatory and Planetarium, College Hill, Armagh BT61 9DG, Ireland \\ 7 Departamento de Astronomía, Universidad de Chile, Casilla 36-D, Santiago, Chile
}

Received 7 June 2016 / Accepted 24 October 2017

\begin{abstract}
Aims. We aim to reveal the morphology, chemical composition, kinematics, and to establish the main processes prevalent in the gas at the footpoints of the giant molecular loops (GMLs) in the Galactic center region.

Methods. Using the 22-m Mopra telescope, we mapped the M-3.8+0.9 molecular cloud, placed at the footpoints of a GML, in 3-mm range molecular lines. To derive the molecular hydrogen column density, we also observed the ${ }^{13} \mathrm{CO}(2-1)$ line at $1 \mathrm{~mm}$ using the 12-m APEX telescope. From the $3 \mathrm{~mm}$ observations 12 molecular species were detected, namely $\mathrm{HCO}^{+}, \mathrm{HCN}, \mathrm{H}^{13} \mathrm{CN}, \mathrm{HNC}, \mathrm{SiO}$, $\mathrm{CS}, \mathrm{CH}_{3} \mathrm{OH}, \mathrm{N}_{2} \mathrm{H}^{+}$, $\mathrm{SO}, \mathrm{HNCO}$, OCS, and $\mathrm{HC}_{3} \mathrm{~N}$.

Results. Maps revealing the morphology and kinematics of the M-3.8+0.9 molecular cloud in different molecules are presented. We identify six main molecular complexes. We derive fractional abundances in 11 selected positions of the different molecules assuming local thermodynamical equilibrium.

Conclusions. Most of the fractional abundances derived for the M-3.8+0.9 molecular cloud are very similar over the whole cloud. However, the fractional abundances of some molecules show significant difference with respect to those measured in the central molecular zone (CMZ). The abundances of the shock tracer SiO are very similar between the GMLs and the CMZ. The methanol emission is the most abundant species in the GMLs. This indicates that the gas is likely affected by moderate $\sim 30 \mathrm{~km} \mathrm{~s}^{-1}$ or even high velocity $\left(50 \mathrm{~km} \mathrm{~s}^{-1}\right)$ shocks, consistent with the line profile observed toward one of the studied position. The origin of the shocks is likely related to the flow of the gas throughout the GMLs towards the footpoints.
\end{abstract}

Key words. ISM: molecules - Galaxy: center - ISM: clouds

\section{Introduction}

The central regions of galaxies interact and exchange matter and radiation with their surroundings. This interaction strongly affects and modifies the physical properties and the chemistry in their nuclear regions. Due to its proximity, the central region of the Milky Way allows detailed high resolution studies of the role of a variety of phenomena, namely magnetic loops (Fukui et al. 2006), galactic winds (Bland-Hawthorn \& Cohen 2003), gas accretion by for example, a barred Galactic model (Binney et al. 1991).

The molecular component of the Galactic center (GC) region (i.e., the inner $\sim 1 \mathrm{kpc}$ of the Galaxy in the context of this work) is composed by a large molecular complex in the central $\sim 500 \mathrm{pc}$ known as the central molecular zone (CMZ, Morris \& Serabyn 1996), and several molecular clouds with high CO luminosity and large velocity dispersion (Bitran et al. 1997) outside the

\footnotetext{
* OPRA and APEX final data cubes (FITS) are only available at the CDS via anonymous ftp to cdsarc.u-strasbg.fr $(130.79 .128 .5)$ or via
}

http://cdsarc.u-strasbg.fr/viz-bin/qcat?J/A+A/613/A42
CMZ from $l \sim-6^{\circ}$ to $l \sim 6^{\circ}$ and $b \sim-1^{\circ}$ to $b \sim 2^{\circ}$ (Fig. 1) that are also placed in the GC region. There are many molecular line emission surveys of the CMZ (e.g., Jones et al. 2012), but few of them cover the molecular clouds beyond the CMZ. Bitran et al. (1997) observed a region of $-12^{\circ}<l<12^{\circ}$, $-2^{\circ}<b<2^{\circ}$ in $\mathrm{CO}(1-0)$, identifying five large velocity width and high $\mathrm{CO}$ luminosity clumps outside the CMZ located in the GC. Fukui et al. (2006) extended the coverage of maps in this line to a larger latitude range with better spatial resolution and identified huge loop structures in the negative velocity range from $-180 \mathrm{~km} \mathrm{~s}^{-1}$ to $-40 \mathrm{~km} \mathrm{~s}^{-1}$ with filamentary structure with a width of $\sim 30 \mathrm{pc}$ and a length of $\sim 400 \mathrm{pc}$ and heights of $\sim 2^{\circ}$ from the Galactic plane. These features are coherent in velocity, with velocity gradients of $0.2-0.35 \mathrm{~km} \mathrm{~s}^{-1} \mathrm{pc}^{-1}$. Fukui et al. (2006) proposed that these giant molecular loops (GMLs) placed in the GC are formed by a magnetic buoyancy caused by a Parker instability. According to the model presented in Fukui et al. (2006), the gas of the loops would flow down their sides, along the magnetic field lines, and join with the gas layer of the Galactic plane, generating shock fronts at the footpoints of the loops. The presence of shocked gas is supported by the 


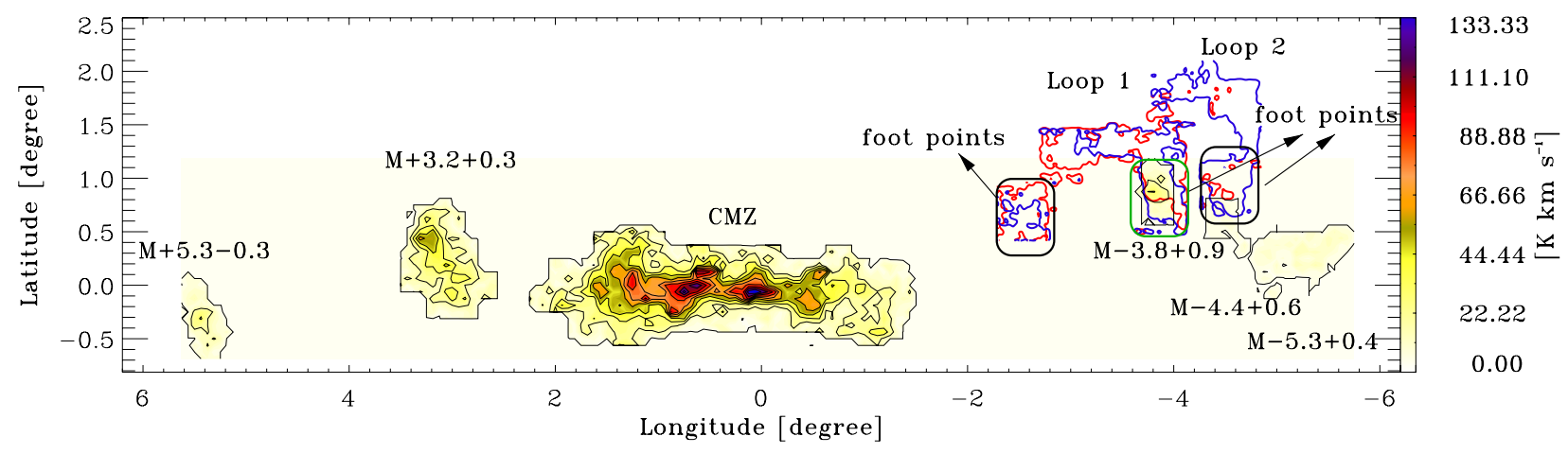

Fig. 1. Overview of the large scale GC region showing the features discussed in this work. The CMZ and five molecular clouds are indicated by the $\mathrm{HCO}^{+}(1-0)$ emission from Riquelme et al. (2010b). Loops 1 and 2 are plotted in the $\mathrm{CO}(2-1)$ emission from Kudo et al. (2011). Loop 1 is integrated in the velocity range -180 to $-90 \mathrm{~km} \mathrm{~s}^{-1}$ (blue contour), and loop 2 from -90 to $-40 \mathrm{~km} \mathrm{~s}^{-1}$ (red contour). The footpoints are indicated with the black ellipse. The green ellipse shows the $\mathrm{M}-3.8+0.9$ molecular cloud studied in this work.

broad velocity features of $\sim 40$ to $80 \mathrm{~km} \mathrm{~s}^{-1}$ width observed by Bitran et al. (1997) and Fukui et al. (2006).

Additional evidence of shocked gas at the footpoints, comes from a survey of the GC region in $\mathrm{HCO}^{+}(1-0), \mathrm{H}^{13} \mathrm{CO}^{+}(1-0)$, and $\mathrm{SiO}(2-1)$ lines (Riquelme et al. 2010b). The mapped area $\left(-5^{\circ} .75<l<5^{\circ} .63\right.$ and $\left.-0^{\circ} .7<b<1^{\circ} .35\right)$ includes the CMZ and the five clumps observed by Bitran et al. (1997) mentioned above. They found an enhancement of the $\mathrm{SiO}$ emission (an archetypical tracer of shocked gas) at the footpoint zones with respect to $\mathrm{HCO}^{+}$. This strongly suggests the presence of shocks (Martín-Pintado et al. 1992, 1997).

Despite there are still no confirmatory magnetic field measurements in these features, multi-transitional $\mathrm{CO}$ observations towards both the footpoints and the complete loops, and magnetohydrodynamical simulations support the GMLs scenario. Table 1 summarizes the characteristics of the GMLs. Torii et al. (2010a) studied in detail the footpoint of the GMLs towards $l \sim 356^{\circ}$ (hereafter M-3.8+0.9 molecular cloud). They identified and analyzed several features including two "U-shapes", which they propose to be formed by the merging of two downward, that is, to lower latitude flows between two loops as predicted by magneto-hydrodynamics numerical simulations (Takahashi et al. 2009; Machida et al. 2009). Fujishita et al. (2009) discovered the "loop 3", placed in the positive-velocity range in $l \simeq 354^{\circ}-359^{\circ}$, and Fukui et al. (2006) proposed the existence of other GMLs connecting molecular clouds with large velocity widths at $l \sim 1^{\circ} .3,3^{\circ} .2$ (Bania et al. 1986), and $5^{\circ} .3$.

Kaneda et al. (2012) found that the polycyclic aromatic hydrocarbon $(\mathrm{PAH})$ infrared emission at $9 \mu \mathrm{m}$ is spatially correlated with the loops; however, it is suppressed in the footpoints as compared with the IRAS $100 \mu \mathrm{m}$ emission. This suggested the destruction of PAHs relative to sub-micron dust grains by shocks.

Isotope studies (Riquelme et al. 2010a) suggest that gas has been accreted towards the footpoint of the loops, and metastable inversion transitions of the ammonia (Riquelme et al. 2013) revealed high kinetic temperatures $(>90 \mathrm{~K})$ in the footpoints of the loop at $l \sim 5^{\circ} .2$. Figure 1 presents an overview of the large scale GC region, showing the CMZ, the five molecular clouds outside the CMZ, and the location of the GMLs discussed in this paper.

This paper presents high angular resolution mapping of 3-mm molecular lines toward the $\mathrm{M}-3.8+0.9$ molecular cloud, placed at the footpoints of the molecular loops discovered by
Fukui et al. (2006) in the GC. These observations allow us to derive the morphology, chemistry and the kinematics of both the quiescent and the shocked gas at small spatial scales of order 1 pc. The projected distance from $\mathrm{Sgr} \mathrm{A}^{*}$ of the $\mathrm{M}-3.8+0.9$ molecular cloud is $564 \mathrm{pc}$, assuming a distance of $8.5 \mathrm{kpc}$ (the IAU recommended value).

\section{Observations and data reduction}

\subsection{Mopra observations and data reduction}

The observations were carried out using the 22-m Mopra telescope during September 2008 and August 2009. Located in the southern hemisphere, and due to its high angular resolution and wide-bandwidth spectrometer, the Mopra telescope offered an excellent possibility of establishing the chemical abundances of the molecular gas at the footpoints of the GC loops. We used the digital mode filter bank MOPS in broadband mode, covering $8 \mathrm{GHz}$ of bandwidth simultaneously in four $2.2 \mathrm{GHz}$ sub-bands, each of them with 8192 channel spaced by $0.27 \mathrm{MHz}$. Two polarizations were measured simultaneously.

We mapped a selected region of the $\mathrm{M}-3.8+0.9$ molecular cloud (Riquelme et al. 2010b) using the on-the-fly (OTF) mapping mode (Ladd et al. 2005; Mangum et al. 2007). Observations of tiles of $5^{\prime} \times 5^{\prime}$ size with overlaps of $30^{\prime \prime}$ were used to cover the complete region. The complete maps covered the region of $356^{\circ} .06<l<356^{\circ} .29$ and $0^{\circ} .645<b<1^{\circ} .1$. We used position switching mode with the off position placed at $(l, b)=\left(356^{\circ} .375,1^{\circ} .5\right)$, which was checked as being free of emission, and observed in symmetric mode (one off per OTF scan). The spacing between scan rows was $12^{\prime \prime}$, and each tile of $5 \times 5 \operatorname{arcmin}^{2}$ took $55 \mathrm{~min}$ to complete. To establish pointing parameter corrections we observed before each map the $\mathrm{SiO}$ maser source AH Sco. The spectra were read out with two seconds of integration time. The system temperature was calibrated with a noise diode and a hot and cold load (paddle) every $30 \mathrm{~min}$. We observed two frequency setups, one centered at $89.41 \mathrm{GHz}$ and the other at $99.72 \mathrm{GHz}$ covering the ranges between 85.275 and $93.555 \mathrm{GHz}$ and from 95.585 to $103.866 \mathrm{GHz}$.

The data were reduced using the LIVEDATA and GRIDZILLA packages. LIVEDATA is the processing software used to apply system temperature calibration, bandpass calibration, heliocentric correction, spectral smoothing and to write out the data in sdfits (Garwood 2000) format. GRIDZILLA is a 
Table 1. Summary of the characteristics of the giant molecular loops.

\begin{tabular}{lccccc}
\hline \hline Loop & $\begin{array}{c}\text { Longitude range } \\
\text { [Degree] }\end{array}$ & $\begin{array}{c}\text { Latitude range } \\
{[\text { Degree] }}\end{array}$ & $\begin{array}{c}\text { Velocity range } \\
{\left[\mathrm{km} \mathrm{s}^{-1}\right]}\end{array}$ & $\begin{array}{c}\text { Mass } \\
M_{\odot}\end{array}$ & Footpoint location \\
\hline Loop 1 & $355.8^{\circ}-358.0^{\circ}$ & $0.5^{\circ}-1.6^{\circ}$ & -180 to -90 & $7-12 \times 10^{5}$ & $(l, b) \sim\left(356^{\circ}, 1^{\circ}\right)$ and $(l, b) \sim\left(357.4^{\circ}, 0.8^{\circ}\right)$ \\
Loop 2 & $355.2^{\circ}-356.6^{\circ}$ & $0.6^{\circ}-2.2^{\circ}$ & -90 to -40 & $8-16 \times 10^{5}$ & $(l, b) \sim\left(355.4^{\circ}, 0.8^{\circ}\right)$ and $(l, b) \sim\left(356.1^{\circ}, 0.8^{\circ}\right)$ \\
Loop 3 & $354^{\circ}-359^{\circ}$ & $0^{\circ}-2^{\circ}$ & $30-160$ & $3 \times 10^{3}$ & $(l, b) \sim\left(355.5^{\circ}, 0.7^{\circ}\right),(l, b) \sim\left(359^{\circ}, 0.6^{\circ}\right)$ \\
\hline
\end{tabular}

regridding software package to convert the sdfits files to the FITS data cube (Jones et al. 2008). A first order polynomial baseline was subtracted with LIVEDATA, and the data were regridded with GRIDZILLA into data cubes, using a Gaussian smoothing interpolation.

The final spatial resolution of the data cubes is between $49^{\prime \prime}$ and $51^{\prime \prime}$ at 115 and $86 \mathrm{GHz}$ respectively, which is obtained after convolution of the Mopra beam width of $33 \pm 3^{\prime \prime}$ at $115 \mathrm{GHz}$ and $36 \pm 3^{\prime \prime}$ from 86 to $100 \mathrm{GHz}$, as measured using Jupiter in 2004 (Ladd et al. 2005), with a Gaussian of 36" full width half maximum (FWHM). This FWHM for the Gaussian size improves the signal-to-noise ratio of the data, albeit with a modest loss in spatial resolution. We calibrated the data in the main beam brightness temperature $\left(T_{\mathrm{mb}}\right)$ scale. The main beam efficiency of Mopra varies between 0.49 at $86 \mathrm{GHz}$ and 0.42 at $115 \mathrm{GHz}$. However, to convert to $T_{\mathrm{mb}}$ we used the values for the extended beam efficiency which are more appropriate for the extended emission of the $\mathrm{GC}$ region $(0.65$ at $86 \mathrm{GHz}$ and 0.55 at $115 \mathrm{GHz}$ ) (Ladd et al. 2005). The spectral resolution of the data is $269.5 \mathrm{kHz}\left(0.94-0.78 \mathrm{~km} \mathrm{~s}^{-1}\right)$. One data cube per molecular line was made. The size of the pixel is 15 arc sec in the final cube. We produced 13 data cubes for the detected molecules in the Mopra survey (see Table 2). In five of those data cubes (CS, $\mathrm{SiO}, \mathrm{HC} 3 \mathrm{~N}-10-9, \mathrm{H} 13 \mathrm{CN}, \mathrm{CH} 3 \mathrm{OH})$, it was necessary to subtract third order baselines for $\sim 20 \%$ of the data using MADCUBAIJ ${ }^{1}$ software.

\subsection{APEX observations and data reduction}

The $J=2-1$ rotational transition of ${ }^{13} \mathrm{CO}$ was mapped using the 12-m Atacama Pathfinder EXperiment (APEX) telescope (Güsten et al. 2006), covering a similar region to that of the Mopra observations (see Fig. 3). The observations were carried out on 24 June, and 1, 2, and 3 July 2014 under the APEX project code M-093.F-008-2014 using the APEX-1 (SHIFI) receiver (Vassilev et al. 2008) and the eXtended bandwidth Fast Fourier Transform Spectrometer (XFFTS) backend (Klein et al. 2012). OTF position-switching observing mode was used, with a close off position slightly contaminated with ${ }^{13} \mathrm{CO}$ emission $\left(\alpha(\mathrm{J} 2000)\right.$ : $17 \mathrm{~h} 33 \mathrm{~m} 13.0 \mathrm{~s}, \delta(\mathrm{J} 2000)$ : $\left.-31^{\circ} 30^{\prime} 13^{\prime \prime} .9\right)$ mainly at the velocity range from -10 to $30 \mathrm{~km} \mathrm{~s}^{-1}$ with an intensity peak $T_{\mathrm{mb}}=0.6 \mathrm{~K}, 100$ to $110 \mathrm{~km} \mathrm{~s}^{-1}$ with an intensity peak of $T_{\mathrm{mb}}=0.2 \mathrm{~K}$, and in much less amount from -80 to $-70 \mathrm{~km} \mathrm{~s}^{-1}$ and from -50 to $-20 \mathrm{~km} \mathrm{~s}^{-1}$ with $T_{\mathrm{mb}}<0.1 \mathrm{~K}$. This contamination was corrected later with observations against a clean off position (RA(J2000): 17h30m48.0s, DEC(J2000): $\left.-31^{\circ} 11^{\prime} 48^{\prime \prime} .1\right)$. This observing strategy ensured flat baselines across the map. The pointing was checked every $1.5-2 \mathrm{~h}$ on IRAS17150-3224; corrections smaller than $2^{\prime \prime}$ were determined. The system temperature $\left(T_{\text {sys }}\right)$ ranged from 125 to $204 \mathrm{~K}$, with an average value of $155 \mathrm{~K}$. The calibration was done using the

1 http://www.cab.inta-csic.es/madcuba standard APEX calibration procedure, with an estimated error of $\sim 10 \%$.

The data were reduced using the CLASS package from the GILDAS software ${ }^{2}$. The antenna temperature $\left(T_{\mathrm{A}}^{*}\right)$ was converted to $T_{\mathrm{mb}}$ using the Ruze formula ${ }^{3}$ with $B_{0}=0.69$ and $\sigma=0.19$ for an extended source (see Appendix B). All spectra were taken into account since the observed rms noise was in all cases lower than $1.5 \times$ the theoretical noise (Ao et al. 2013; Ginsburg et al. 2016) ${ }^{4}$. Third order polynomial baselines were subtracted for the OTF mapping observations of the M-3.8+0.9 molecular cloud, and a fifth order polynomial baselines for the position switching observations in the reference position. Then, each individual spectrum for the mapping was combined with the reference position spectrum using the accumulate function in CLASS with an equal weighting. In this way, the baseline timedependence is removed. Since line widths $>10 \mathrm{~km} \mathrm{~s}^{-1}$ were expected, the final spectra were smoothed with the box function in CLASS to reach a final velocity resolution of $1.04 \mathrm{~km} \mathrm{~s}^{-1}$ which is more than enough to resolve all the kinematic structures of this molecular cloud. The data were regridded in equatorial coordinates and then converted to Galactic coordinates for comparison with the Mopra data using standard CLASS routines. The average root-mean-square (rms) noise for the spectra in the data cube is $127 \mathrm{mK}$ at a velocity resolution of $\approx 1 \mathrm{~km} \mathrm{~s}^{-1}$. The final data cube was corrected for additional baseline subtraction using MADCUBAIJ software in small regions when needed (second or third order polynomial base line subtraction in the $30 \%$ of the data).

\section{Results}

Following the list of most prominent $3 \mathrm{~mm}$ wavelength molecular lines observed in Sgr B2 by Jones et al. (see their Table 2 in 2008), we made one data cube per molecule ${ }^{5}$. Tables 2 and 3 show the molecules and the rms noise values reached in each molecular line cube. The results are presented both in the main text and in Appendices A-D.

\subsection{Morphology}

The morphology and velocity structure are illustrated by the $\mathrm{HCN}$ and ${ }^{13} \mathrm{CO}$ molecular lines, which show the most intense emission among all detected molecules. Figures 2 and 3 show: (I) the integrated brightness temperature maps of $\mathrm{HCN}$ and ${ }^{13} \mathrm{CO}(2-1)$, for the observed region of $\mathrm{M}-3.8+0.9$, in the velocity range from $v_{\mathrm{LSR}}=-140$ to $v_{\mathrm{LSR}}=-20 \mathrm{~km} \mathrm{~s}^{-1}$, (II) the

\footnotetext{
2 http://www.iram.fr/IRAMFR/GILDAS

$3 B_{\text {eff }}(\lambda)=B_{0} \times \exp \left(-(4 \times \pi \times \sigma / \lambda)^{2}\right)$.

4 Where the theoretical noise was estimated from $T_{\text {sys }} / \sqrt{\Delta v \times T_{\text {exp }}}$ for the OTF observations and $2 \times T_{\text {sys }} / \sqrt{\Delta v \times T_{\exp }}$ for the position switching observations in the reference position (Mangum et al. 2007). 5 All data cubes publicly available.
} 
Table 2. Imaged molecular transitions, their rms noise level $\left(T_{\mathrm{A}}^{*}\right)$ and the main beam efficiency.

\begin{tabular}{lcccc}
\hline \hline Molecule & Transition & $\begin{array}{c}\text { Rest. freq. } \\
{[\mathrm{GHz}]}\end{array}$ & $\begin{array}{c}\mathrm{rms}^{c} \\
{[\mathrm{mK}]}\end{array}$ & $\eta_{\mathrm{mb}}$ \\
\hline $\mathrm{H}^{13} \mathrm{CN}$ & $1-0^{a}$ & 86.340 & 54 & 0.65 \\
$\mathrm{SiO}$ & $2-1 v=0$ & 86.847 & 51 & 0.65 \\
$\mathrm{HNCO}$ & $4(0,4)-3(0,3)$ & 87.925 & 53 & 0.64 \\
$\mathrm{HCN}$ & $1-0^{a}$ & 88.632 & 58 & 0.64 \\
$\mathrm{HCO}^{+}$ & $1-0$ & 89.188 & 34 & 0.64 \\
$\mathrm{HNC}$ & $1-0^{a}$ & 90.664 & 38 & 0.63 \\
$\mathrm{HC}_{3} \mathrm{~N}$ & $10-9$ & 90.980 & 40 & 0.63 \\
$\mathrm{~N}_{2} \mathrm{H}^{+}$ & $1-0^{a}$ & 93.174 & 38 & 0.62 \\
$\mathrm{CH}_{3} \mathrm{OH}$ & $2-_{K}^{a}$ & 96.74 & 50 & 0.61 \\
$\mathrm{OCS}$ & $8-7$ & 97.300 & 57 & 0.61 \\
$\mathrm{CS}$ & $2-1$ & 97.980 & 50 & 0.61 \\
$\mathrm{SO}$ & $3(2)-2(1)$ & 99.300 & 58 & 0.60 \\
$\mathrm{HC}$ & $11-10$ & 100.08 & 53 & 0.60 \\
${ }^{13} \mathrm{CO}$ & $2-1^{b}$ & 220.398 & $127^{d}$ & 0.67 \\
\hline
\end{tabular}

Notes. ${ }^{(a)}$ Have fine or hyperfine structure transitions. ${ }^{(b)}$ Observed with the APEX telescope. ${ }^{(c)}$ The spectral resolution of the data is $0.27 \mathrm{MHz}$ which corresponds to a velocity resolution from $0.78-0.94 \mathrm{~km} \mathrm{~s}^{-1}$ depending of the frequency of the species. ${ }^{(d)}$ Velocity resolution of $1.04 \mathrm{~km} \mathrm{~s}^{-1}$.

Table 3. Non-detected molecular transitions and their rms noise level in a $0.27 \mathrm{MHz}$ wide channel.

\begin{tabular}{lccc}
\hline \hline Molecule & Transition & $\begin{array}{c}\text { Rest. freq. } \\
{[\mathrm{GHz}]}\end{array}$ & $\begin{array}{c}\text { rms } \\
{[\mathrm{mK}]}\end{array}$ \\
\hline $\mathrm{CH}_{3} \mathrm{CCH}$ & $5-4^{a}$ & 85.457 & 48 \\
$\mathrm{HOCO}^{+}$ & $4(0,4)-3(0,3)$ & 85.530 & 56 \\
$\mathrm{SO}$ & $2(2)-1(1)$ & 86.093 & 43 \\
$\mathrm{H}^{13} \mathrm{CO}^{+}$ & $1-0$ & 86.754 & 53 \\
$\mathrm{HN}^{13} \mathrm{C}$ & $1-0^{a}$ & 87.091 & 55 \\
$\mathrm{CCH}$ & $1-0^{a}$ & 87.328 & 56 \\
$\mathrm{CH}{ }_{3} \mathrm{CN}$ & $5-4^{a}$ & 91.979 & 50 \\
${ }^{13} \mathrm{CS}$ & $2-1$ & 92.494 & 50 \\
$\mathrm{C}^{34} \mathrm{~S}$ & $2-1$ & 96.410 & 46 \\
$\mathrm{CH}_{3} \mathrm{OH}$ & $2(1,1)-1(1,0) \mathrm{A}-$ & 97.582 & 77 \\
$\mathrm{NH}_{2} \mathrm{CN}$ & $5(1,4)-4(1,3)$ & 100.63 & 63 \\
$\mathrm{H}_{2} \mathrm{CS}$ & $3(1,3)-2(1,2)$ & 101.48 & 79 \\
$\mathrm{CH}_{3} \mathrm{CCH}$ & $6-5^{a}$ & 102.530 & 77 \\
$\mathrm{H}_{2} \mathrm{CS}$ & $3(0,3)-2(0,2)$ & 103.04 & 54 \\
\hline
\end{tabular}

Notes. ${ }^{(a)}$ Have fine or hyperfine structure transitions.

longitude-velocity map integrated over the whole observed latitude range, and (III) the latitude-velocity map integrated in the whole observed longitude range. Figures 4 and 5 show the integrated brightness temperature of the molecular emission in velocity intervals of $10 \mathrm{~km} \mathrm{~s}^{-1}$ in $\mathrm{HCN}$, and in Figs. A.1 and A. 2 we show the ${ }^{13} \mathrm{CO}(2-1)$ emission. The $\mathrm{M}-3.8+0.9$ cloud has a narrow line width emission at positive velocities $\left(\sim 120 \mathrm{~km} \mathrm{~s}^{-1}\right)$ at $(l, b) \sim\left(356^{\circ} .25,0^{\circ} .70\right)$, which can be seen in the $\mathrm{HCN}$ emission (Fig. 5). Its position coincides with that of an ultra-compact H II region (Caswell \& Haynes 1987), but this source has a radial velocity of $113 \mathrm{~km} \mathrm{~s}^{-1}$, and therefore is not associated to the footpoint as noted by Torii et al. (2010a). Therefore this source will not be further discussed in this work. This source was only partially covered by the ${ }^{13} \mathrm{CO}$ observation. There are also several molecular clouds showing positive velocities in the ${ }^{13} \mathrm{CO}$ emission (Fig. A.2). The emission at $\sim 70-90 \mathrm{~km} \mathrm{~s}^{-1}$ could be associated to the far-3 kpc arm (Dame \& Thaddeus 2008) and the emission at $90-130 \mathrm{~km} \mathrm{~s}^{-1}$ could be associated to the $135 \mathrm{~km} \mathrm{~s}^{-1}$ arm (Bania 1980), as noted by Riquelme et al. (2010b), which are not associated with the loops 1 and 2, and they will not be discussed in this work.

Figures 4 and A.1 show the presence of a velocity gradient from higher to lower velocities in the north-western to the southeastern direction. Four main velocity components are identified: from -140 to $-70 \mathrm{~km} \mathrm{~s}^{-1}$, from -70 to $-40 \mathrm{~km} \mathrm{~s}^{-1}$, from -40 to $-20 \mathrm{~km} \mathrm{~s}^{-1}$, and from -20 to $20 \mathrm{~km} \mathrm{~s}^{-1}$. In Figs. C.4-C.13 we show the integrated intensities maps between $-140 \mathrm{~km} \mathrm{~s}^{-1}$ and $-20 \mathrm{~km} \mathrm{~s}^{-1}$ as well as the integrated intensities maps in the four velocity ranges for all the detected transitions listed in Table 2 . In these velocity ranges, six main molecular complexes are identified and indicated in Fig. 6 on the HCN maps. These complexes were identified by visual inspection and may be spatially correlated if the $\mathrm{M}-3.8+0.9$ cloud has a velocity gradient of $2 \mathrm{~km}^{-1} \mathrm{pc}^{-1}$. For an easy comparison, the ${ }^{13} \mathrm{CO}$ emission is also plotted in this figure. The velocity structure is also shown in the latitude-velocity domain integrated in longitude steps of $108^{\prime \prime}$ (from Figs. D.1-D.13). In those figures we can see the two U-shapes identified by Torii et al. (2010b) (see Sect. 5.4).

\subsection{Complex 3}

In the velocity range from -140 to $-70 \mathrm{~km} \mathrm{~s}^{-1}$, the most prominent feature is the Complex 3, which shows an elongated structure perpendicular to the Galactic plane with an abrupt sharp intensity decrease towards the eastern edge. This complex also contains two intensity peaks that are detected in most of the molecules (see figures in Appendix $C$ ) at $(l, b)=\left(356^{\circ} .22,0^{\circ} .91\right)$ and $(l, b)=\left(356^{\circ} .21,0^{\circ} .84\right)$. The ${ }^{13} \mathrm{CO}, \mathrm{HC}_{3} \mathrm{~N}, \mathrm{~N}_{2} \mathrm{H}^{+}$, and $\mathrm{SO}$ molecular emissions have the intensity peak in the north, in contrast with the $\mathrm{HCN}, \mathrm{HCO}^{+}$, and $\mathrm{CS}$ emissions which have the intensity peak in the south. Some molecules only appear in the north (e.g., $\mathrm{HNCO}, \mathrm{N}_{2} \mathrm{H}^{+}$, SO, and $\mathrm{HC}_{3} \mathrm{~N}$ ).

\subsection{Complexes 1, 2, 4, 5, and 6}

In the velocity range from -70 to $-40 \mathrm{~km} \mathrm{~s}^{-1}$, the Complexes 1, 2, 4, and 6 can be identified. Complex 1 has a very large line width $\left(-110\right.$ to $\left.0 \mathrm{~km} \mathrm{~s}^{-1}\right)$. It is also strong in $\mathrm{HCN}$ emission, while in $\mathrm{HCO}^{+}$emission the intensity is weaker but still cover the same velocity range. In the other molecules, the emission shows two kinematic components with the intensity peak at $\sim-40 \mathrm{~km} \mathrm{~s}^{-1}$. This complex is very prominent in the $\mathrm{HCN}, \mathrm{HCO}^{+}, \mathrm{CS}, \mathrm{HNC}$, and $\mathrm{CH}_{3} \mathrm{OH}$ emission (see from Figs. C.4-C.13). Although the Complex 2 is not visible in all the detected molecules, it is the region which presents the largest line width in the M-3.8+0.9 molecular cloud, as can be seen in the Appendix $\mathrm{C}$ which is consistent with previous works (see, Torii et al. 2010b,a). The Complex 4 appears to be connecting Complexes 3 and 6 . This complex is very prominent in ${ }^{13} \mathrm{CO}, \mathrm{CS}$, $\mathrm{HCO}^{+}, \mathrm{HCN}$, and HNC. It is remarkable that Complex 6 also shows an elongated structure perpendicular to the Galactic plane similar to that observed for Complex 3 . This complex also shows an abrupt sharp intensity decrease towards the eastern edge. In the velocity range from -40 to $-20 \mathrm{~km} \mathrm{~s}^{-1}$, we can see the Complex 5 which is an elongated feature, parallel to the Galactic plane. In the last velocity range, from -20 to $20 \mathrm{~km} \mathrm{~s}^{-1}$, the emission shows a shell-like structure which appears over the 
complete molecular cloud and is clearly seen in the ${ }^{13} \mathrm{CO}, \mathrm{HCO}^{+}$ and HNC maps (Figs. 6, C.5, and C.6). As can be seen in the channel maps of ${ }^{13} \mathrm{CO}$ plots (Fig. A.1), this feature is indeed narrower in velocity, from -10 to $10 \mathrm{~km} \mathrm{~s}^{-1}$. This emission could be associated to local gas along the line of sight and since it is probably not associated to the GMLs, this feature will not be discussed in this work.

\section{Analysis}

\section{1. ${ }^{13} \mathrm{CO}(2-1)$ emission. $\mathrm{H}_{2}$ column density estimate}

Since the ${ }^{13} \mathrm{CO}$ emission is optically thin (see optical depth estimations for $\mathrm{CO}$ in Torii et al. 2010a) and the critical density is relatively low $\left(n_{\text {crit }} \sim 10^{4} \mathrm{~cm}^{-3}\right)$, this molecule is a good tracer of the total $\mathrm{H}_{2}$ column density using a proper conversion factor $\left(N\left(\mathrm{H}_{2}\right)=N\left({ }^{13} \mathrm{CO}\right) \times\left[{ }^{13} \mathrm{CO} / \mathrm{H}_{2}\right]\right)$. Since we only have one transition, we assumed local thermodynamical equilibrium (LTE) at a excitation temperature $\left(T_{\mathrm{ex}}\right)$ of $10 \mathrm{~K}$ to derive the column density, $N$ :

$N=\frac{8 \pi k v^{2} Q\left(T_{\mathrm{ex}}\right)}{c^{3} A_{i j} g_{\mathrm{u}} h} \exp \left(\frac{E_{\mathrm{u}}}{k T_{\mathrm{ex}}}\right) \frac{1}{\left(1-\frac{J\left(T_{\mathrm{bg}}\right)}{J\left(T_{\mathrm{ex}}\right)}\right)} \int T_{b} \mathrm{~d} v$,

where $k$ is the Boltzmann constant, $v$ the frequency of the transition, $h$ the Planck constant, $Q(T)$ the partition function at the assumed excitation temperature, $g_{\mathrm{u}}$ the upper state degeneracy, $A_{i j}$ the Einstein coefficient, $E_{\mathrm{u}}$ the energy of the upper state, and $J(T)=\frac{h v}{k}\left(\frac{1}{(\exp ((h v) /(k T))-1}\right)$ is the source function at a temperature $T$. The molecular parameters were taken from the Cologne Database for Molecular Spectroscopy (CDMS) catalog (Müller et al. 2005, 2001), and $T_{\mathrm{bg}}=2.73 \mathrm{~K}$ is the cosmic background radiation temperature. If we use the $T_{\mathrm{ex}}=40 \mathrm{~K}$ as derived by the multi-J transition study of ${ }^{12} \mathrm{CO}$ by Torii et al. (2010a), the $\mathrm{N}\left({ }^{13} \mathrm{CO}\right)$ increase by less than $10 \%$ (see Fig. A.3). Thus, we decided to use $10 \mathrm{~K}$ which is consistent with our estimations in Riquelme et al. (2013) for CS, and with the discussion in Sect. 5.1.

For our calculations, we assumed an abundance ratio $\mathrm{CO} / \mathrm{H}_{2}$ of $10^{-4}$ (Frerking et al. 1982). This abundance ratio was also used by Rodríguez-Fernández et al. (2001), Dahmen et al. (1998) and Hüttemeister et al. (1998) for their large scale studies of the GC, which cover the complete $\mathrm{CMZ}$ and the Bania's clump2. As noted by Hüttemeister et al. (1998), Farquhar et al. (1994) showed that this ratio is stable against a possibly enhanced cosmic ray flux to the GC. Riquelme et al. (2010a) derived a high ${ }^{12} \mathrm{C} /{ }^{13} \mathrm{C}$ isotopic value (34-73) in several positions in this molecular cloud which is higher than the typical values (20-25) found in the GC region (see, e.g., Langer \& Penzias 1990; Wilson \& Matteucci 1992). Therefore, the value of 53 was used, corresponding to the typical value found in the $4 \mathrm{kpc}$ molecular ring (Wilson \& Rood 1994), which was also used by Torii et al. (2010a), Kudo et al. (2011) and Riquelme et al. (2013) in the GMLs regions. This translates into a $\left[{ }^{13} \mathrm{CO} / \mathrm{H}_{2}\right]$ conversion factor of $1.9 \times 10^{-6}$.

\subsection{Comparison of the emission between different molecules}

To compare and quantify the differences and similarities between the emission distribution throughout the M-3.8+0.9 molecular cloud in the different detected molecules, we performed a principal component analysis and we derived the fractional abundances in selected positions.

\subsubsection{Principal component analysis}

A principal component analysis (PCA, see, e.g. Heyer \& Schloerb 1997; Shlens 2014; Ungerechts et al. 1997; Lo et al. 2009; Jones et al. 2012) was performed using the most intense molecular lines in Table 2, namely ${ }^{13} \mathrm{CO}, \mathrm{HCN}, \mathrm{HCO}^{+}, \mathrm{HNC}$, $\mathrm{CH}_{3} \mathrm{OH}$, and CS. As mentioned in Sect. 2.1, the Mopra beam varies from $33 \pm 3^{\prime \prime}$ at $115 \mathrm{GHz}$ and $36 \pm 3^{\prime \prime}$ from $86-100 \mathrm{GHz}$. Then, the beam sizes for the molecules used in the PCA analysis are identical within the uncertainties. The resolution of the pixel in all data cubes is $15^{\prime \prime}$. The ${ }^{13} \mathrm{CO}(2-1)$ data was converted to Galactic coordinates using standards class routines using one of the Mopra datacube (HCN) as a pattern, imposing the pixel size of $15^{\prime \prime}$. Therefore, all data cubes used in the PCA have a uniform spatial resolution. To implement the method, a python script with the PCA module ${ }^{6}$ was used in a similar way as Jones et al. (2012), using a covariance matrix method. Because the PCA analysis works with normalized data, we were only able to compare data with a good signal to noise ratio. Since the spectroscopic parameters from the different molecules and ${ }^{13} \mathrm{CO}$, as well as the critical densities are different, it is possible that the different molecules are not tracing the same gas. We used the integrated emission in the velocity range from -140 to $-20 \mathrm{~km} \mathrm{~s}^{-1}$, which covers the complete velocity range corresponding to the $\mathrm{GC}$ region since the emission from -20 to $20 \mathrm{~km} \mathrm{~s}^{-1}$ correspond to local gas in the line of sight. The PCA is also restricted to an area defined using a mask in the HCN emission at a $14-\sigma$ level $\left(13.29 \mathrm{~K} \mathrm{~km} \mathrm{~s}^{-1}\right)$. This threshold was chosen to include all the important features visible in the Figs. C.4-C.13 and excluding the regions with low signal to noise ratio which can add spurious features into the PCA.

The PCA shows that the emission distribution of all the molecular lines studied are closely related. The first three principal components describe the 94, 2, and 1.5 per cent of the variance in the data. Figure 7 shows the results of the PCA. The color scale indicates the correlation between the molecules. A positive value indicates that the emission from these molecules are correlated and the negative values show an anti-correlation. The first principal component axis in Fig. 8 shows that all six molecules are positively correlated. The PCA1 image in Fig. 7 shows emission common to the six molecular lines with a large fraction of the variance (94\%) indicating that all molecular lines are similarly distributed throughout the molecular cloud. The major features are Complexes 2 and 3 which show the largest correlations in the cloud. The PCA2 image shows the major difference among the six remaining molecules. Despite the value of the variance is small $(2 \%)$, these differences are still physically significant because the positive features follow very well the Complex 2 which is dominant in $\mathrm{HCN}$ and $\mathrm{HCO}^{+}$, and the negative features correspond to the north part of the Complexes 3, 4, 5, and 6 where ${ }^{13} \mathrm{CO}, \mathrm{HNC}, \mathrm{CH}_{3} \mathrm{OH}$, and, to to less extent the CS emission are enhanced. The PCA3 has only a minimum value of the variance $(1.5 \%)$ and shows smaller variations mainly between the ${ }^{13} \mathrm{CO}$ and $\mathrm{HNC}$ emissions.

\subsubsection{Spectra towards selected positions}

Using the images of the different PCA previously discussed, we select 11 positions which have an intensity peak in the PCA1, which corresponds to the regions where the most intense molecules are correlated, and peaks in the PCA2 which corresponds to regions with the largest anti correlation between them.

6 http://folk.uio.no/henninri/pca_module 
$\mathrm{K} \mathrm{km} \mathrm{s}^{-1}$

0. 12. 24. 36. 48. 60. 71 .

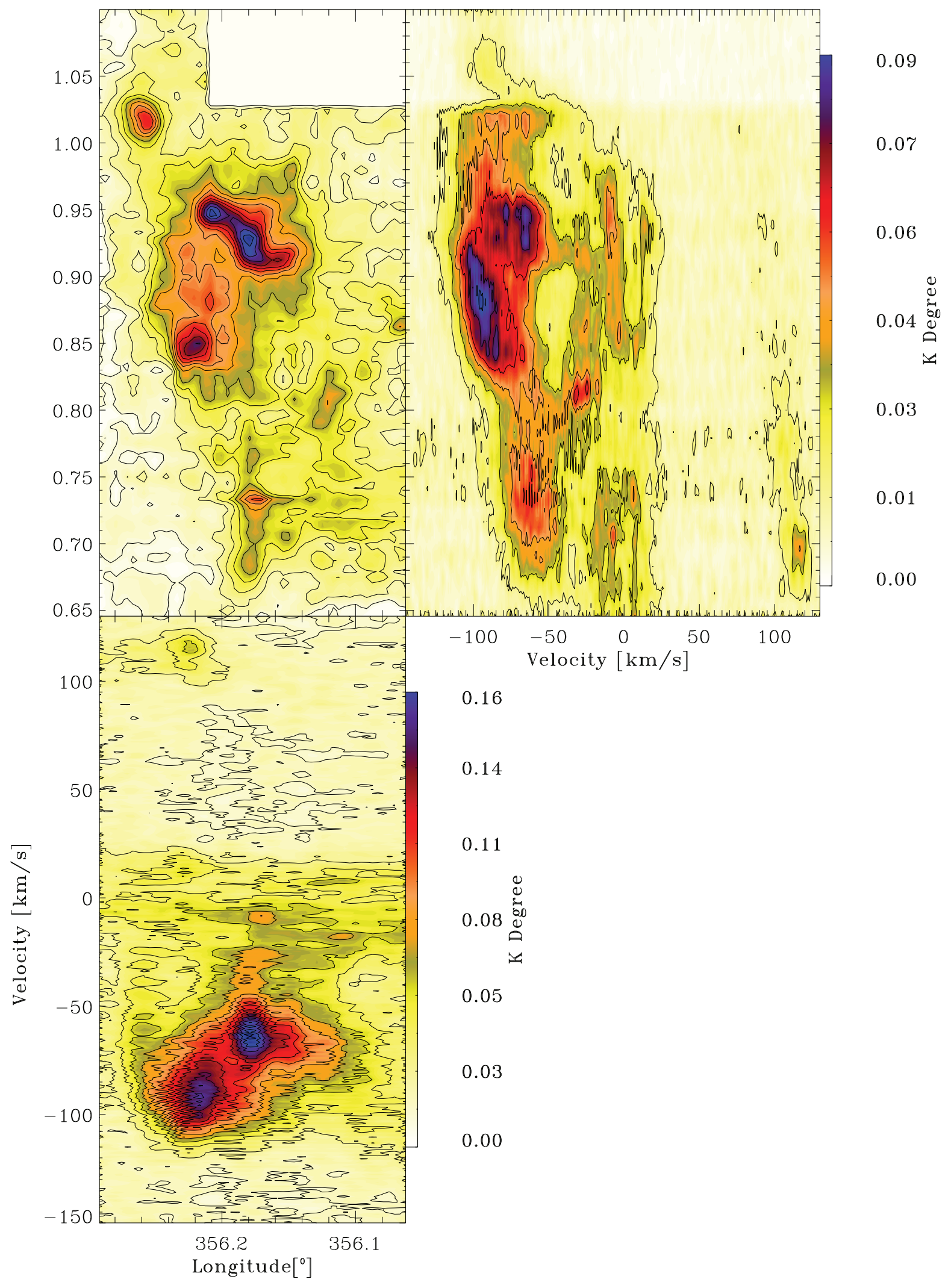

Fig. 2. $\mathrm{HCN}(1-0)$ emission of the $\mathrm{M}-3.8+0.9$ cloud. Left: integrated brightness temperature map in the velocity range from -140 to $-20 \mathrm{~km} \mathrm{~s} \mathrm{~s}^{-1}$. Right: latitude-velocity plot integrated over the whole longitude range (from $l=356.29^{\circ}$ to $356.06^{\circ}$ ). Bottom: longitude-velocity plot integrated over the whole latitude range (from $b=0.64^{\circ}$ to $b=1.10^{\circ}$ ). 
$\left[\mathrm{K}_{\mathrm{km} \mathrm{s}} \mathrm{s}^{-1}\right]$

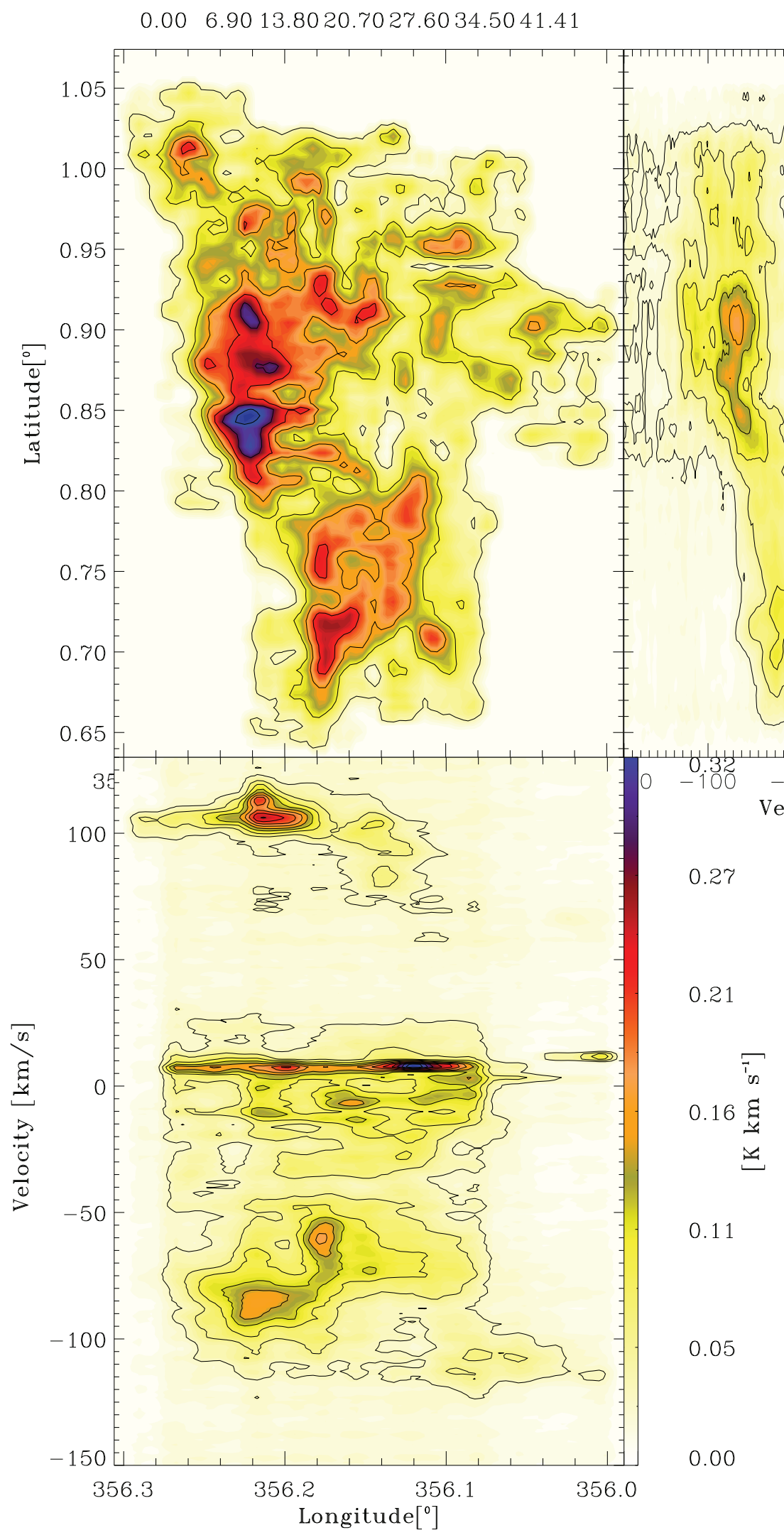

Fig. 3. ${ }^{13} \mathrm{CO}(2-1)$ emission of the $\mathrm{M}-3.8+0.9$ cloud. Left: integrated brightness temperature map in the velocity range from -140 to $-20 \mathrm{~km} \mathrm{~s}{ }^{-1}$. The dashed lines show the mapped region. Right: latitude-velocity plot integrated over the whole longitude range (from $l=356^{\circ}$ to $356^{\circ} .294$ ). Bottom: longitude-velocity plot integrated over the whole latitude range (from $b=0^{\circ} .6423$ to $b=1^{\circ} .051$ ). 
A\&A 613, A42 (2018)

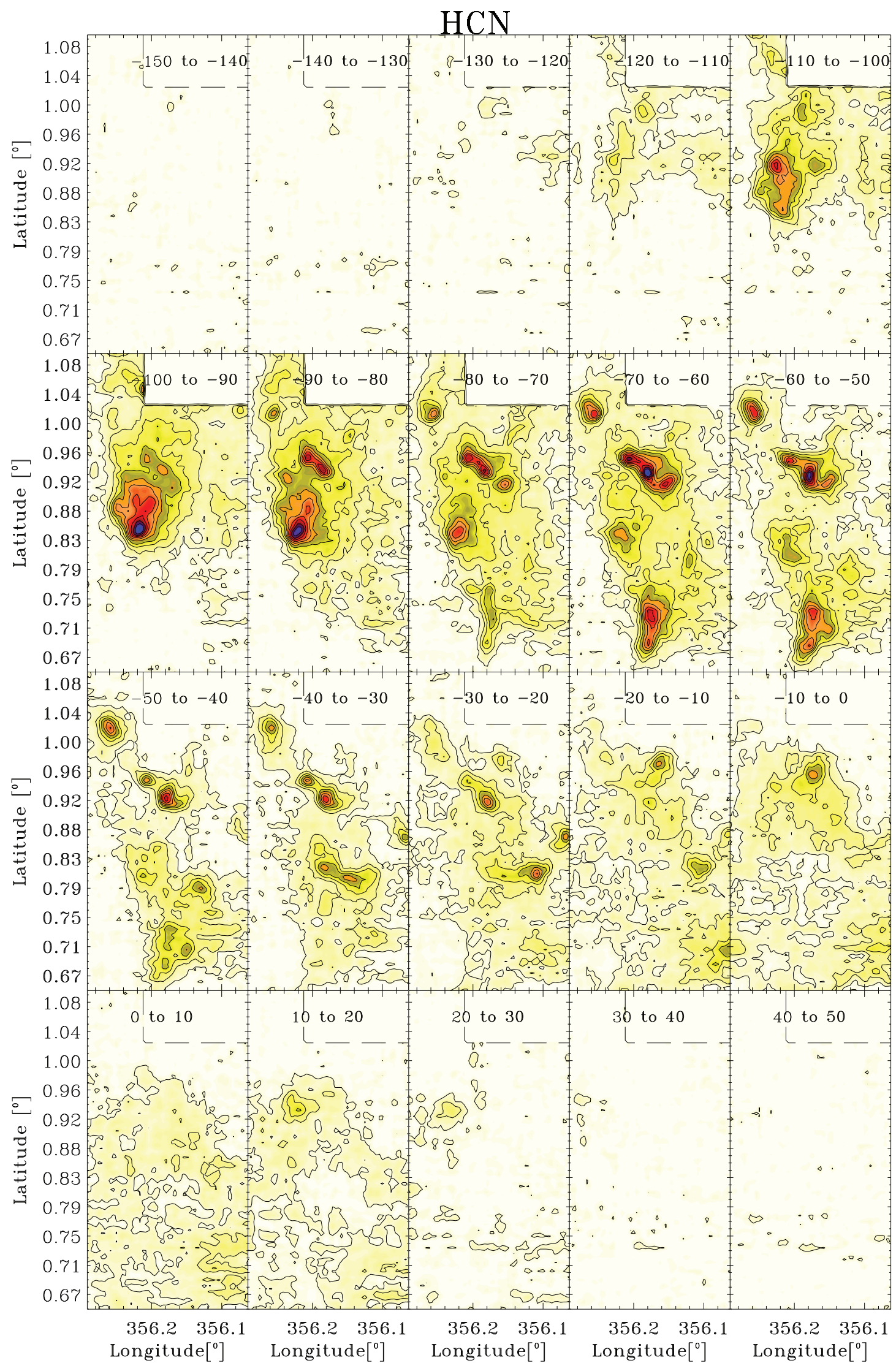

$\left[\mathrm{K} \mathrm{km} \mathrm{s}^{-1}\right]$

\begin{tabular}{lllllll}
\hline 0.00 & 1.53 & 3.06 & 4.58 & 6.11 & 7.64 & 9.16
\end{tabular}

Fig. 4. Integrated brightness temperature of the $\mathrm{M}-3.8+0.9$ molecular cloud in $\mathrm{HCN}(1-0)$ in velocity intervals of $10 \mathrm{~km} \mathrm{~s}^{-1}$. 


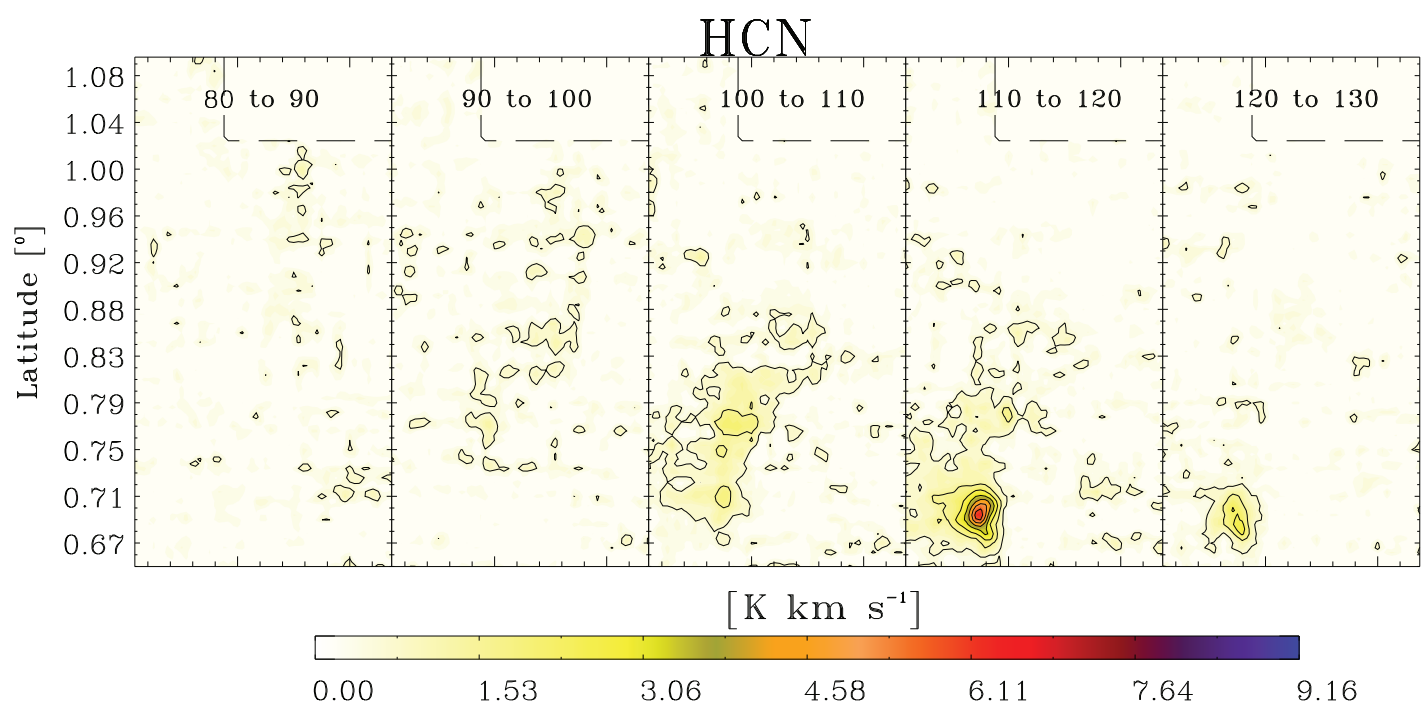

Fig. 5. Integrated brightness temperature of the M-3.8+0.9 molecular cloud in $\mathrm{HCN}(1-0)$ in velocity intervals of $10 \mathrm{~km} \mathrm{~s}^{-1}$.

We selected at least one position per complex (Complexes 1 to 6 as defined in Sect. 3.1), then for example, the position chosen towards the Complex 4 is not spotlighted in any of the PCA results. Because of the high correlation between the molecular transitions, this selection is equivalent to select the peaks in any of the six molecules considered in the PCA analysis. We obtain the integrated spectra in the boxes shown in Fig. 9 in all the detected molecules (Figs. 10 and 11). From those figures, we can see the complex velocity structure in the M-3.8+0.9 molecular cloud, with large velocity widths in all the selected regions. Most of the molecules show a similar line profile within each region, with the clear exception of $\mathrm{HCN}$, which in some regions has the intensity peak at a very different velocity. A clear example is shown in positions 1.a, 2.a, 2.b, and 5.a, with the most extreme case in position 1.a where the profile of $\mathrm{HCN}$ differs completely from the one shown by the other molecules. The differences in the intensity peak of $\mathrm{HCN}$ could be explained by opacity effects since the $\mathrm{N}(\mathrm{HCN}) / \mathrm{N}\left(\mathrm{H}^{13} \mathrm{CN}\right)$ ratio range from 6 to 21 which indicates that the HCN is optically thick. Position 3.b shows a characteristic shock profile (see e.g., Jiménez-Serra et al. 2009), with a prominent wing at the redshifted part of the spectra. The strong shock observed in this position produces a gas acceleration up to $50 \mathrm{~km} \mathrm{~s}^{-1}$. As a good tracer of the column density, the ${ }^{13} \mathrm{CO}$ spectra also clearly show the local gas in the line of sight as narrow emission at $\sim 0 \mathrm{~km} \mathrm{~s}^{-1}$, which is not seen in other molecules. This narrow emission is superposed to a broader one which corresponds to gas in the GC.

\subsubsection{Molecular abundances across the $M-3.8+0.9$ molecular cloud}

The column density for all the detected molecules was derived as indicated in Sect. 3.2. Because we only have one transition for each molecule, we assume LTE at a $T_{\mathrm{ex}}=10 \mathrm{~K}$. If we assume a $T_{\mathrm{ex}}$ of, for example, $24 \mathrm{~K}$ (Jones et al. 2012) column density varies a factor $\sim 1.5$ in most of the molecules considered here, as can be seen in Fig. A.3 with the exception of $\mathrm{CH}_{3} \mathrm{OH}$ which varies a factor of 2.5 , and $\mathrm{HC}_{3} \mathrm{~N}$ (with a factor of 0.4 ). The molecular parameters are taken from the CDMS catalog for all the molecules with the exception of $\mathrm{CH}_{3} \mathrm{OH}$ which are taken from the Jet Propulsion Laboratory (JPL) catalog (Pickett et al. 1998). The column density is derived for the 11 regions defined in Fig. 9 for the velocity range shown in the shadow rectangle in Fig. 10 and 11, and in the Table A.1. The velocity range for each position was chosen to include all the emission for the $\mathrm{M}-3.8+0.9$ cloud avoiding the possible contamination from local gas between -20 to $20 \mathrm{~km} \mathrm{~s}^{-1}$. Table A.1 shows the results. $\mathrm{HC}_{3} \mathrm{~N}$ has two detected rotational transitions. This allows to better estimate the column density and rotational temperature using rotational diagrams (Goldsmith \& Langer 1999). However, because the energy involved of both transitions are very close, the rotational temperature is poorly constrained, and only positions 3.a and 3.b have adequate signal to noise ratio in both transitions. Therefore, to derive the column density of $\mathrm{HC}_{3} \mathrm{~N}$, we used only the (10-9) transition (which is the one with the better signal-to-noise ratio) assuming a temperature of $10 \mathrm{~K}$ like with the others species in this work.

To compare the emission from the different positions, we plot the fractional abundances $\left(X(\mathrm{~mol})=N(\mathrm{~mol}) / N\left(\mathrm{H}_{2}\right)\right.$, where mol corresponds to every detected molecule) normalized by the average value for each molecule. The fractional abundance is computed for the velocity range shown in Table A.1, and Figs. 10 and 11. The plots show in the upper right corner, the average value and the standard deviation of the fractional abundance for each molecule, which is computed using only detected emission and not the upper limits. The error bars corresponds to the estimated $3 \sigma$ uncertainties. Despite the fact that the PCA analysis showed the large correlation between the most intense molecules across the $\mathrm{M}-3.8+0.9$ molecular cloud, Fig. 12 shows that there are significant differences $(3-\sigma)$ between the abundance of different molecules in the selected regions. The fractional abundances are shown in Table 4.

\section{Discussion}

\subsection{Comparison between the molecular fractional abundances in the footpoints and in the $C M Z$}

The fractional abundances derived toward the footpoints of the GMLs are compared with those derived in the CMZ (Table 5). In order to obtain a set of fractional abundances values for the $\mathrm{CMZ}$ as homogeneous as the one presented in this work, we used the Mopra 3-mm data cubes from Jones et al. (2012) to derive the molecular column densities, and for estimating the $\mathrm{H}_{2}$ column 


\section{$\mathrm{HCN}$}

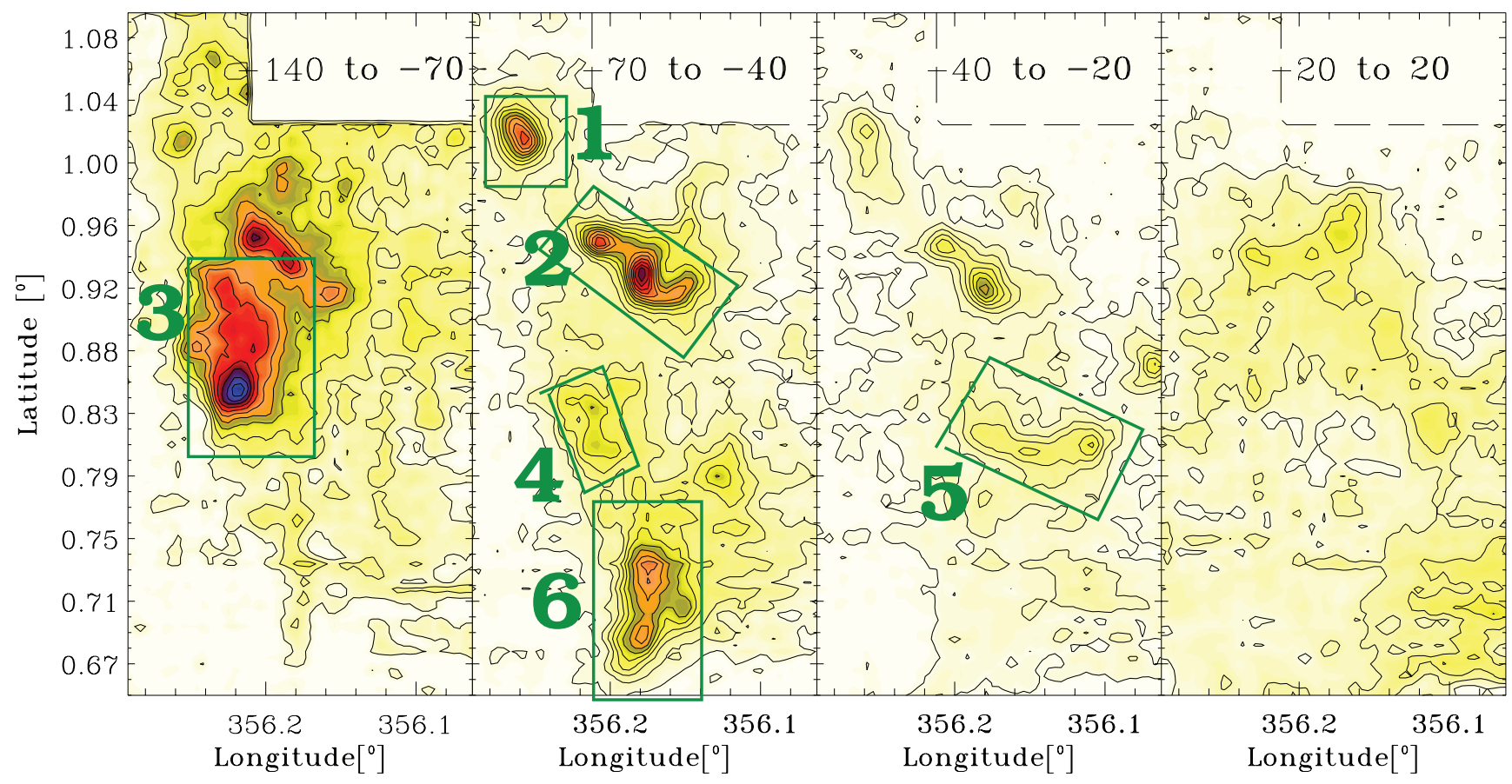

$\left[\mathrm{K} \mathrm{km} \mathrm{s}^{-1}\right]$

\begin{tabular}{|c|c|c|c|c|c|}
\hline 0.00 & 6.88 & ${ }^{13.75} \mathrm{CO}$ & $(20.62)$ & 27.50 & 34.38 \\
\hline
\end{tabular}

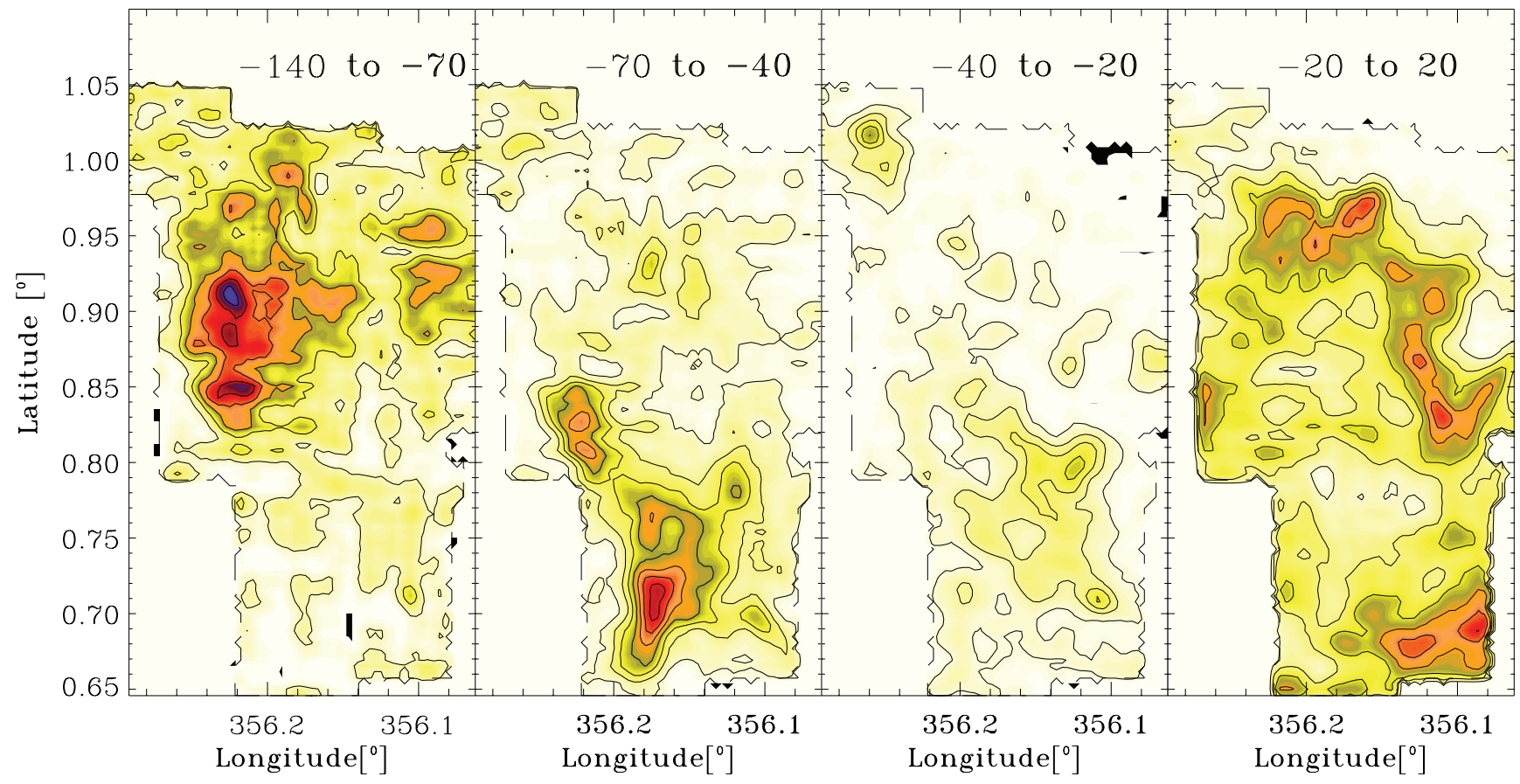

$\left[\mathrm{K} \mathrm{km} \mathrm{s}^{-1}\right]$

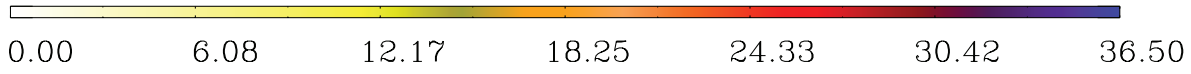

Fig. 6. Position of the six molecular complexes discussed in the text. The green boxes show the complexes discussed in the text. 
PCA 1

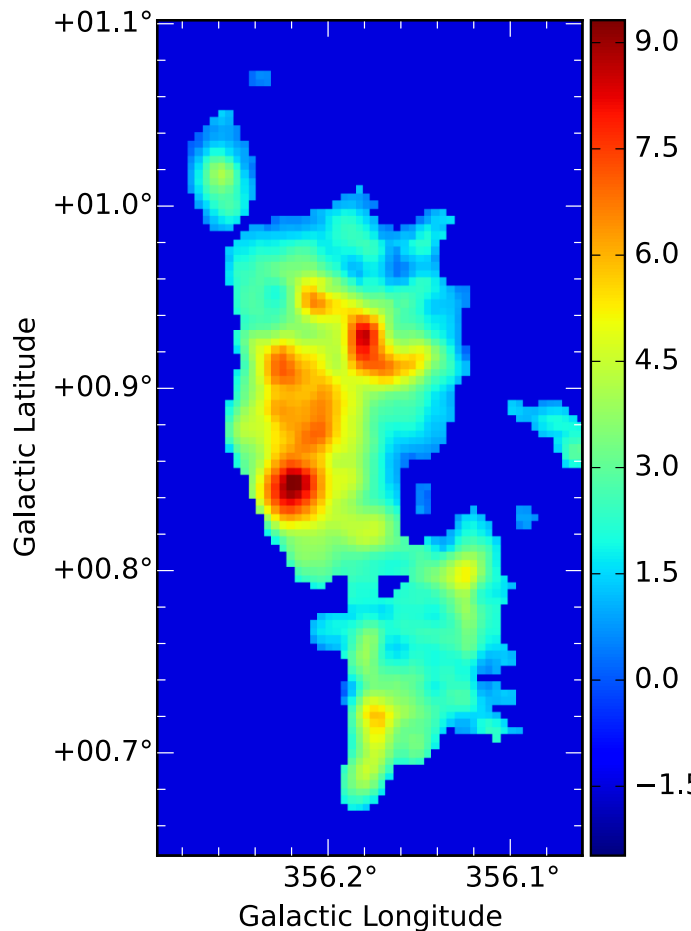

PCA 2

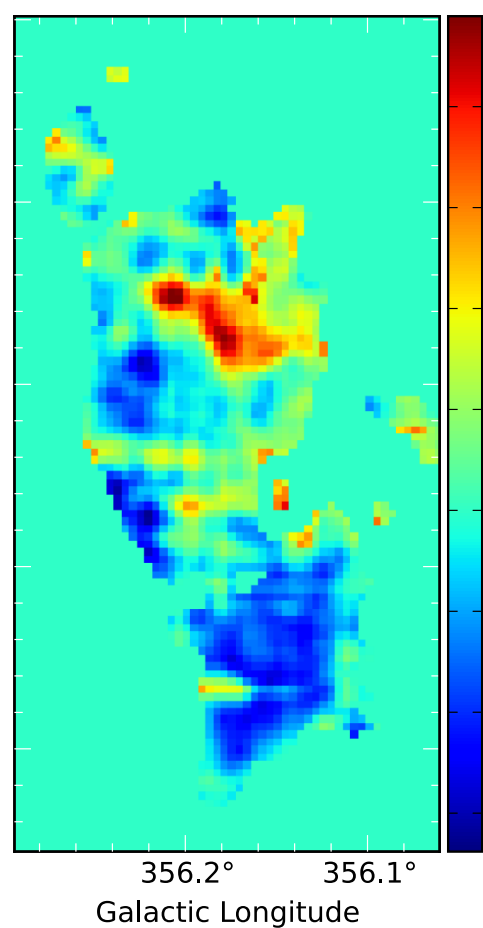

PCA 3

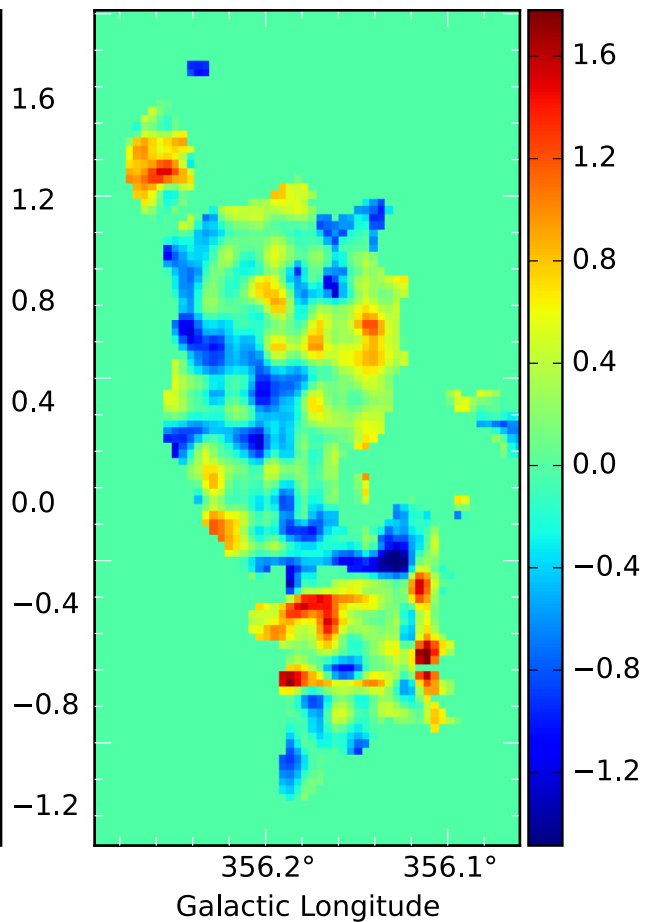

Fig. 7. First three PC of the M-3.8+0.9 molecular cloud (see text in Sect. 4.2.1).

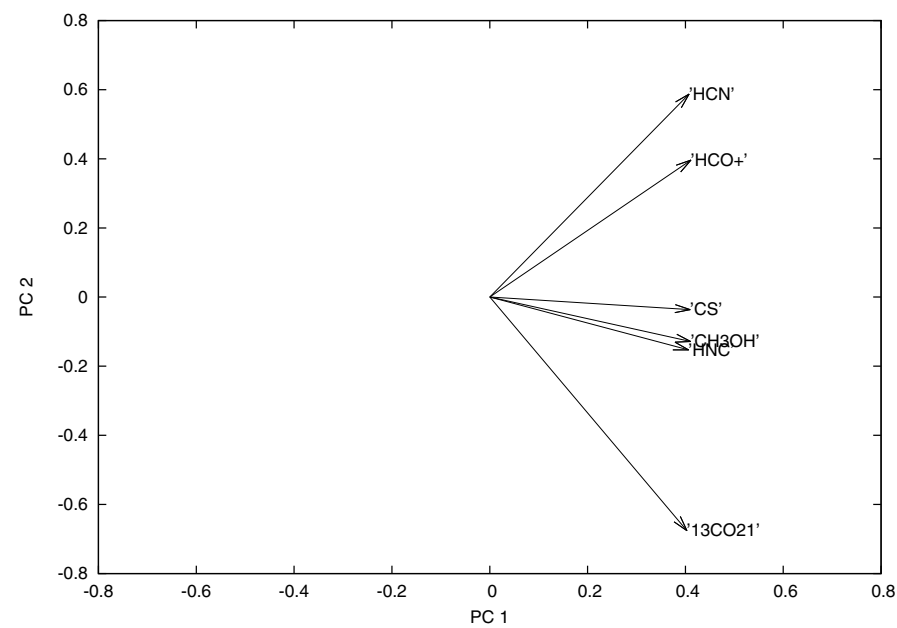

Fig. 8. Eigenvectors of the first three PC.

density, we used the "APEX CMZ SHFI-1 survey" ${ }^{13} \mathrm{CO}(2-1)$ data cube from Ginsburg et al. (2016) ${ }^{7}$. Since the ${ }^{12} \mathrm{C} /{ }^{13} \mathrm{C}$ isotopic value in the CMZ is 24 (Langer \& Penzias 1990), we use a conversion factor $\left[{ }^{13} \mathrm{CO} / \mathrm{H}_{2}\right]$ of $5 \times 10^{-6}$. We selected representative clouds in the CMZ shown in Fig. A.4. The regions were chosen to be of similar size in Fig. $9\left(75^{\prime \prime} \times 75^{\prime \prime}\right)$. The column densities are derived assuming LTE with an excitation temperature of $10 \mathrm{~K}$, using Eq. (1) for the optically thin emission; and for the optically thick emission $\left(\mathrm{HCN}, \mathrm{HCO}^{+}\right.$and HNC), we use the equations in Jones et al. (2012), computing the optical depth along the velocity axis in the same way than in Jones et al. (2012). To assume that the ${ }^{13} \mathrm{CO}$ emission is optically thin is a reasonable approximation as shown by

\footnotetext{
7 Available at http://doi .org/10.7910/DVN/27601.
}

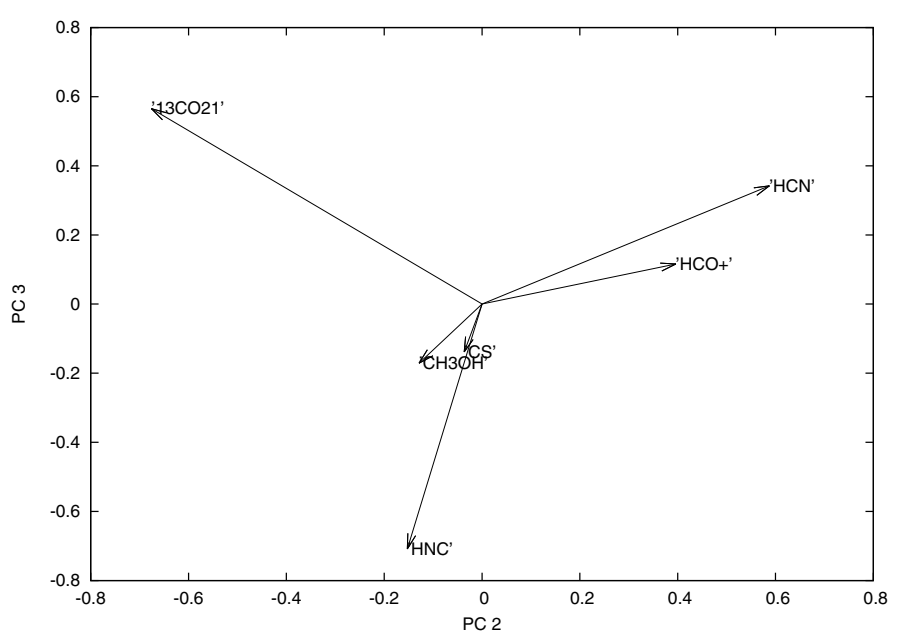

Rodríguez-Fernández et al. (2001) from ${ }^{13} \mathrm{CO}$ and $\mathrm{C}^{18} \mathrm{O}$ $(J=1-0,2-1)$ observations in many sources widespread in the CMZ and also in the clump 2 (Bania et al. 1986). We did not use the $T_{\mathrm{ex}}=24 \mathrm{~K}$ because Jones et al. (2013) found that the excitation temperature for the lower rotational transitions of the molecules that they have 3-mm and 7-mm transitions observations available $\left(\mathrm{SiO}, \mathrm{HNCO}, \mathrm{HC}_{3} \mathrm{~N},{ }^{13} \mathrm{CS}, \mathrm{HOCO}^{+}\right)$was between 2 and $9 \mathrm{~K}$, concluding that the excitation temperature have to be much lower than the kinetic temperature of $30 \mathrm{~K}$. To derive the column density of CS, we use their optically thin isotopomer ${ }^{13} \mathrm{CS}$ and a assumed ${ }^{12} \mathrm{C} /{ }^{13} \mathrm{C}$ isotopic value of 24 . The SO emission from Jones et al. (2012) is not included because they observed a transition not detected by us, and because they only detected it clearly in Sgr B2. Using upper limit information is not meaningful because of the poor baselines of the spectra 
PCA 1

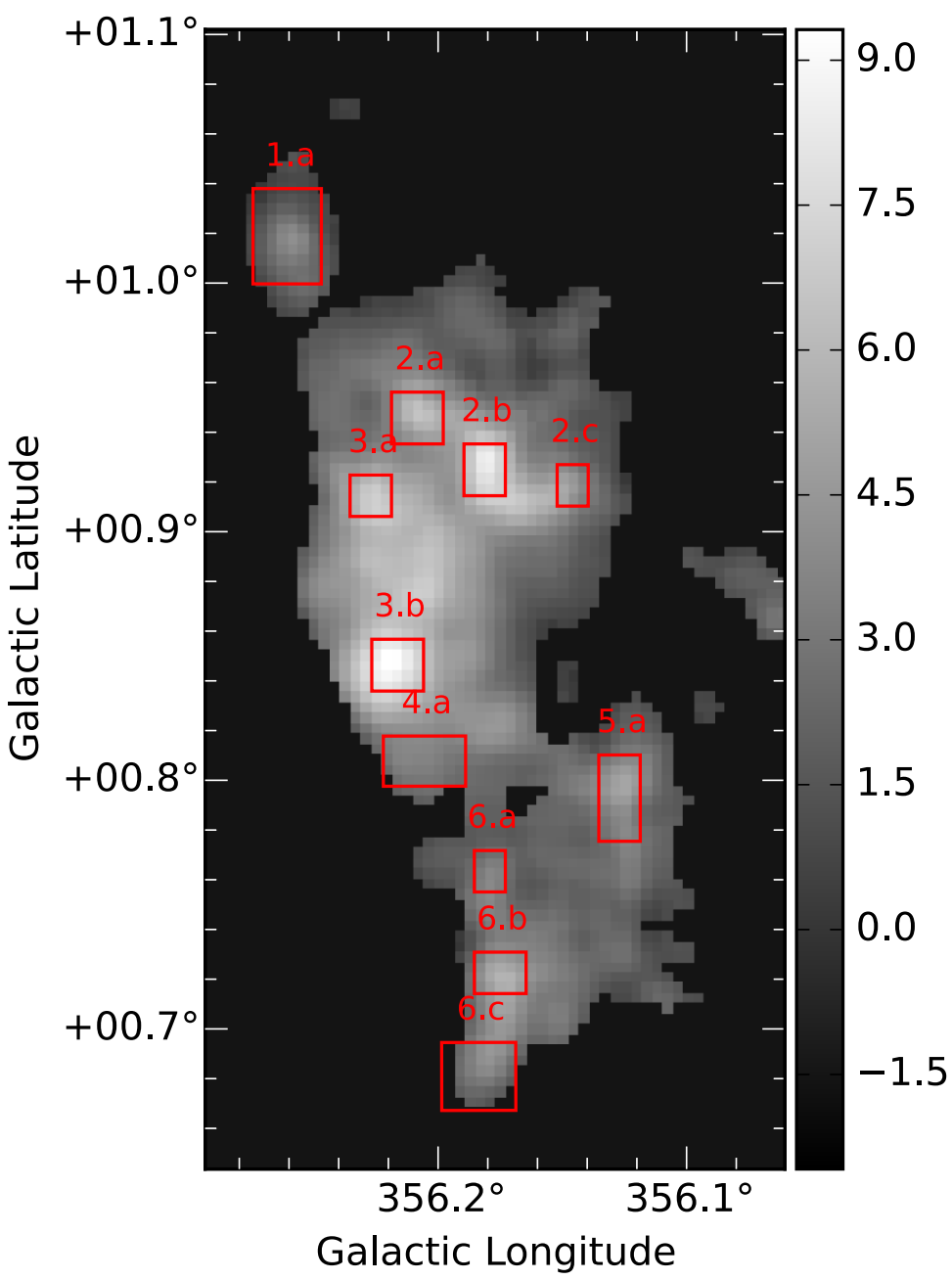

PCA 2

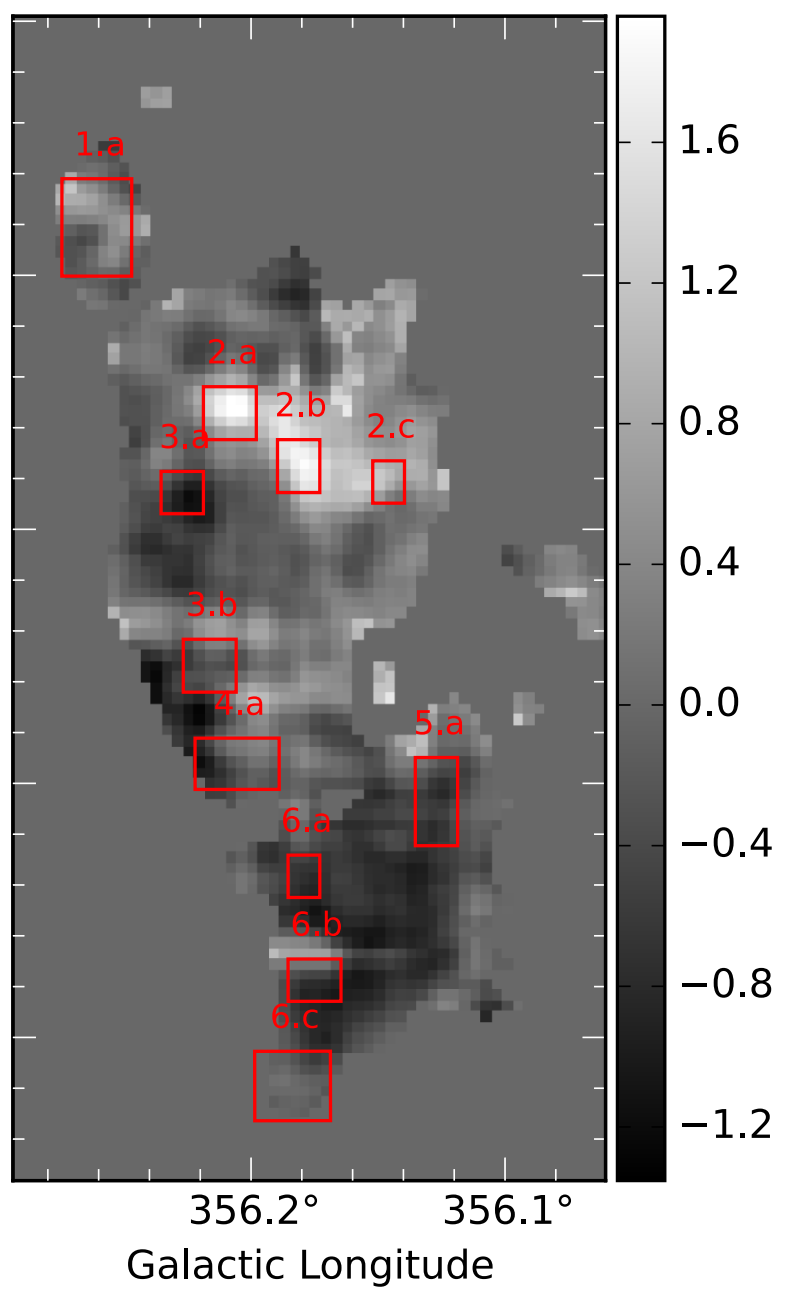

Fig. 9. Selected regions to extract average spectra and to derive fractional abundances of the detected molecules.

in the data cube. Table 5 also includes the fractional abundances derived in selected line-of-sights toward the $\mathrm{CMZ}$ from recent publications covering most of the species observed in this work. The ratio of the fractional abundances between the CMZ (Jones et al. (2012) data) and the GMLs range from a factor 1.1 up to 5.1 for the different molecules. The largest differences are found for $\mathrm{HNCO}, \mathrm{N}_{2} \mathrm{H}^{+}, \mathrm{HNC}$. The smallest differences are found for $\mathrm{SiO}$, with a factor of only 1.1 , which indicate similarities in the chemistry of this molecule in the CMZ and in the footpoints. If we consider the same conversion factor between the CMZ and the $M-3.8+0.9$ cloud (see Sect. 4.1) the ratio of the fractional abundances between the CMZ and the GMLs is 0.5 up to 2 for the different molecules.

\subsection{High velocity shocks}

The species observed in this work provide a set of key molecules to derive the physical and chemical properties of the clouds. Emission from medium and high density tracers is intense and widespread in the M-3.8+0.9 molecular cloud. For example, CS can trace densities of $n>10^{4} \mathrm{~cm}^{-3}$ (Mauersberger \& Henkel 1989). It is only marginally enhanced in UV (Martín et al. 2008) and shock-dominated environments (Requena-Torres et al. 2006). $\mathrm{HCN}, \mathrm{HNC}, \mathrm{HCO}^{+}$with higher critical densities $\left(n \sim 10^{5}-10^{6} \mathrm{~cm}^{-3}\right.$ ) are expected to trace higher density gas rather than diffuse emission from the surrounding lower density cloud. Other molecules such as, e.g. HNCO, can trace gas even denser $\left(n>10^{6} \mathrm{~cm}^{-3}\right.$ Jackson et al. 1984), but the abundance of this molecule is also driven by shock chemistry (see below).

We have three shock tracers in our samples: $\mathrm{SiO}, \mathrm{HNCO}$, and $\mathrm{CH}_{3} \mathrm{OH}$. The enhancement of the $\mathrm{SiO}$ abundance can be explained by the sputtering of grain cores after the passage of magnetohydrodynamics shocks (see Jiménez-Serra et al. 2009, for details of the shocks properties). The $\mathrm{Si}$ or directly the $\mathrm{SiO}$ is released into the gas phase. However, the $\mathrm{SiO}$ emission could also be enhancement by X-rays, as suggested by the correlation between the $6.4 \mathrm{keV} \mathrm{Fe}$ line with the $\mathrm{SiO}$ emission (Martín-Pintado et al. 2000; Amo-Baladrón et al. 2009), and by cosmic-rays (Yusef-Zadeh et al. 2013) as suggested by the correlation with the $74 \mathrm{MHz}$ nonthermal emission. Brogan et al. (2003) present large scale observations of the GC region at $74 \mathrm{MHz}$, and we can see that there is no enhancement of this emission at the position of $\mathrm{M}-3.8+0.9$ cloud. The X-ray scenario requires a population of very small grains to produce the $\mathrm{SiO}$ abundance enhancement, together with a past episode of bright $\mathrm{X}$-ray emission from some source in the GC (Amo-Baladrón et al. 2009). Only a few X-ray sources are detected close to M-3.8+0.9 cloud (Roberts et al. 2001), therefore we do not 
D. Riquelme et al.: Giant molecular loops in the GC region
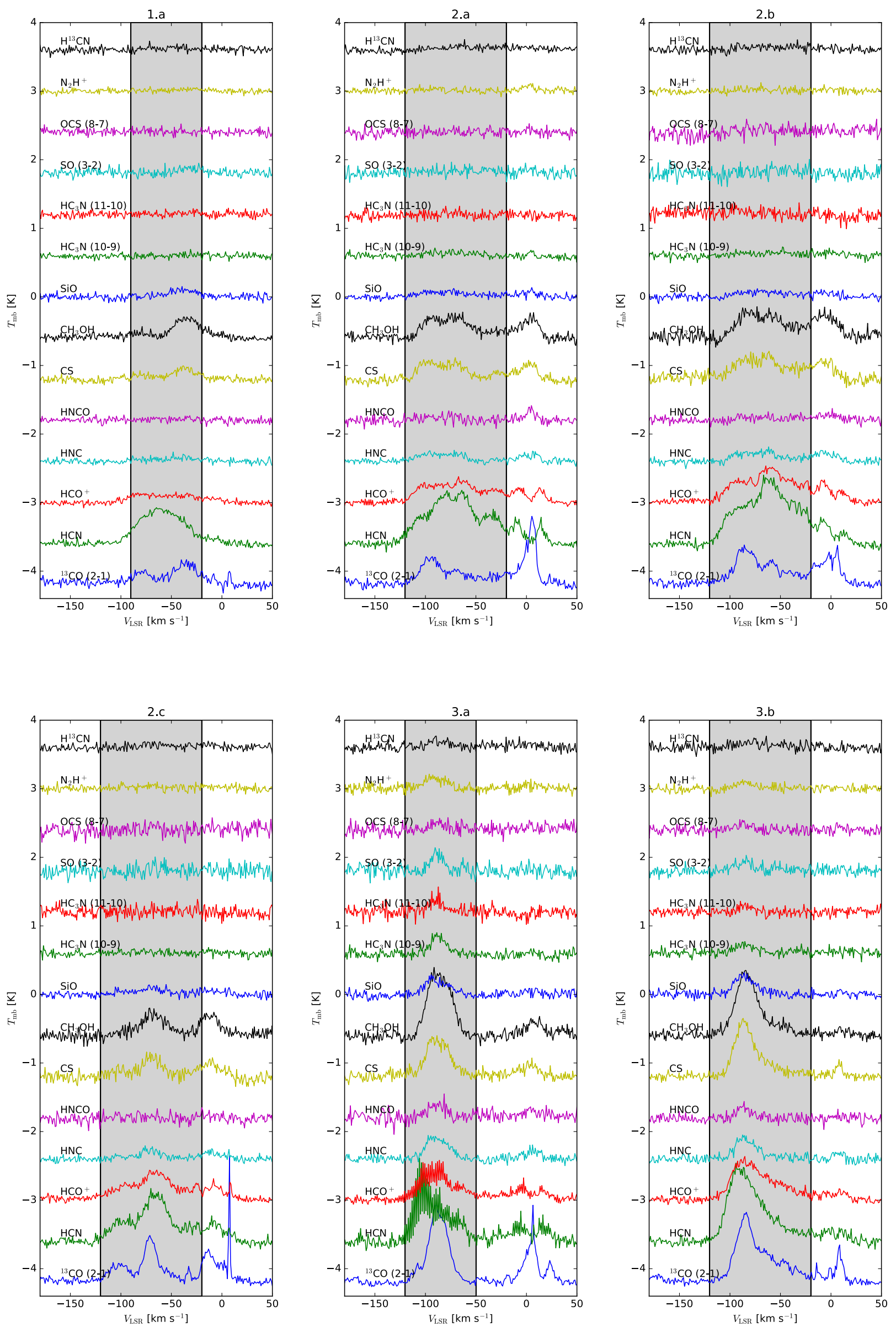

Fig. 10. Average spectra for each box shown in Fig. $9 .{ }^{13} \mathrm{CO}(2-1), \mathrm{HCO}^{+}, \mathrm{HCN}, \mathrm{CH}_{3} \mathrm{OH}, \mathrm{CS}$ spectra are scaled by a factor 0.5 . 

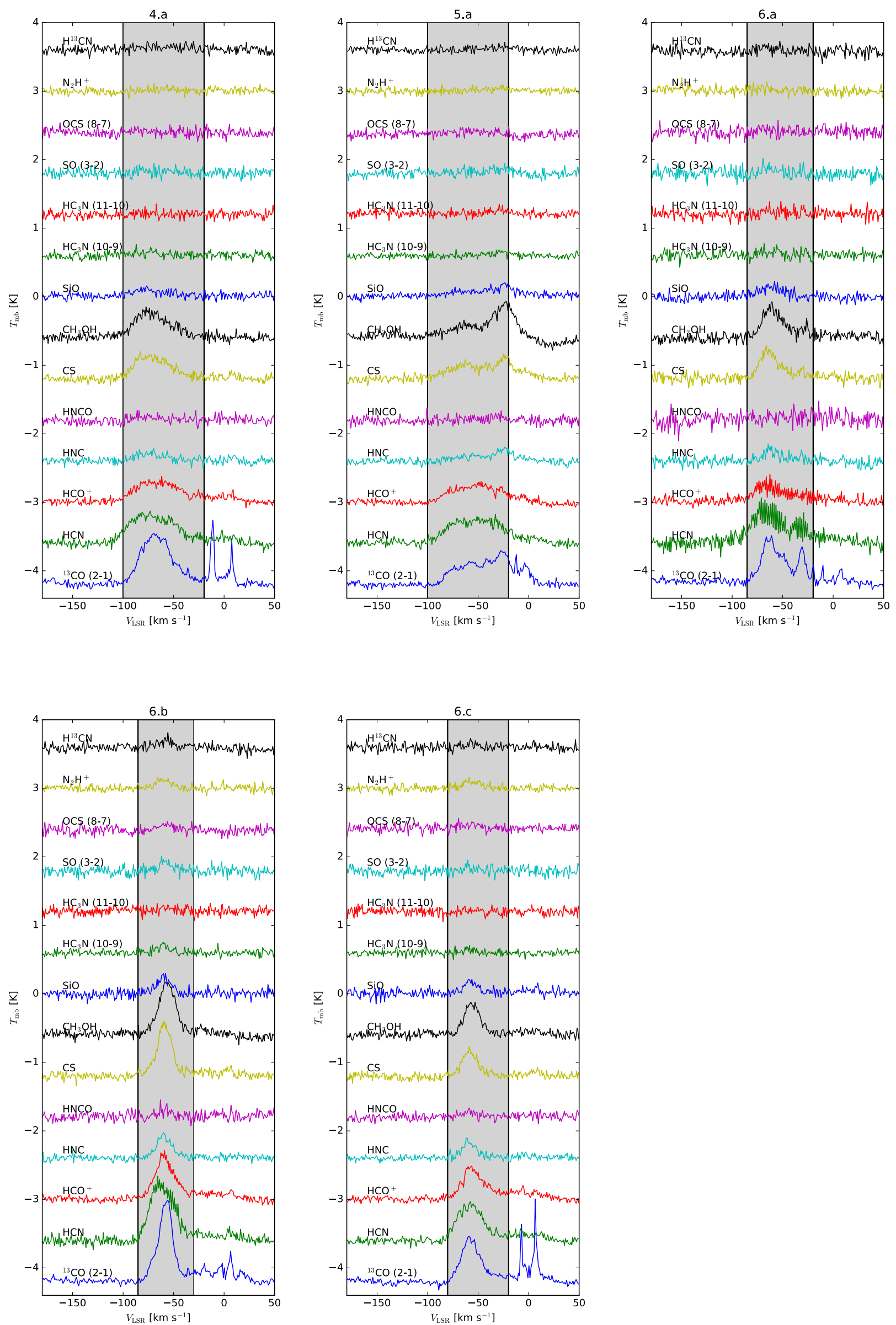

Fig. 11. Average spectra for each box shown in Fig. 9.

A42, page 14 of 37 
D. Riquelme et al.: Giant molecular loops in the GC region
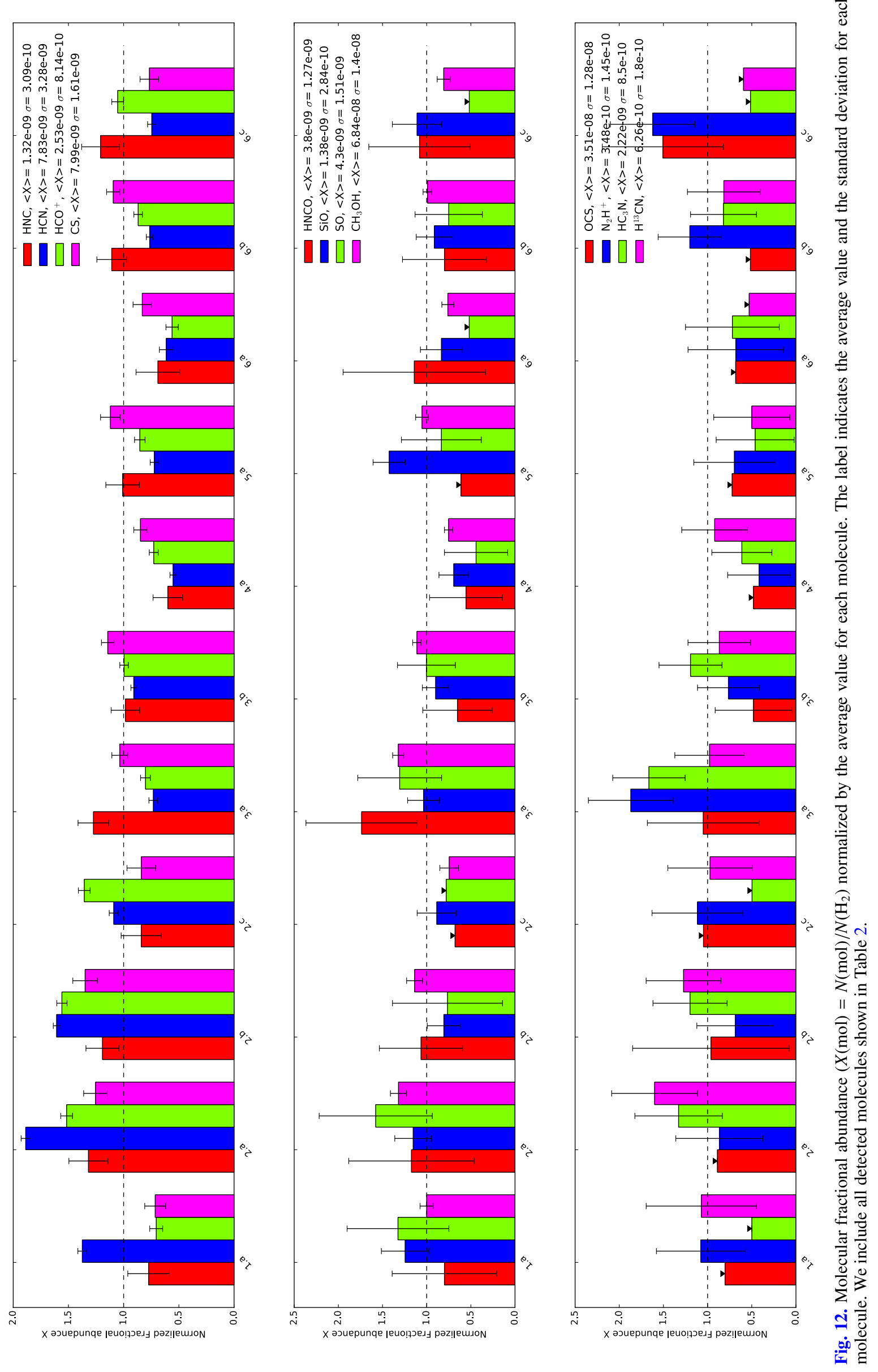


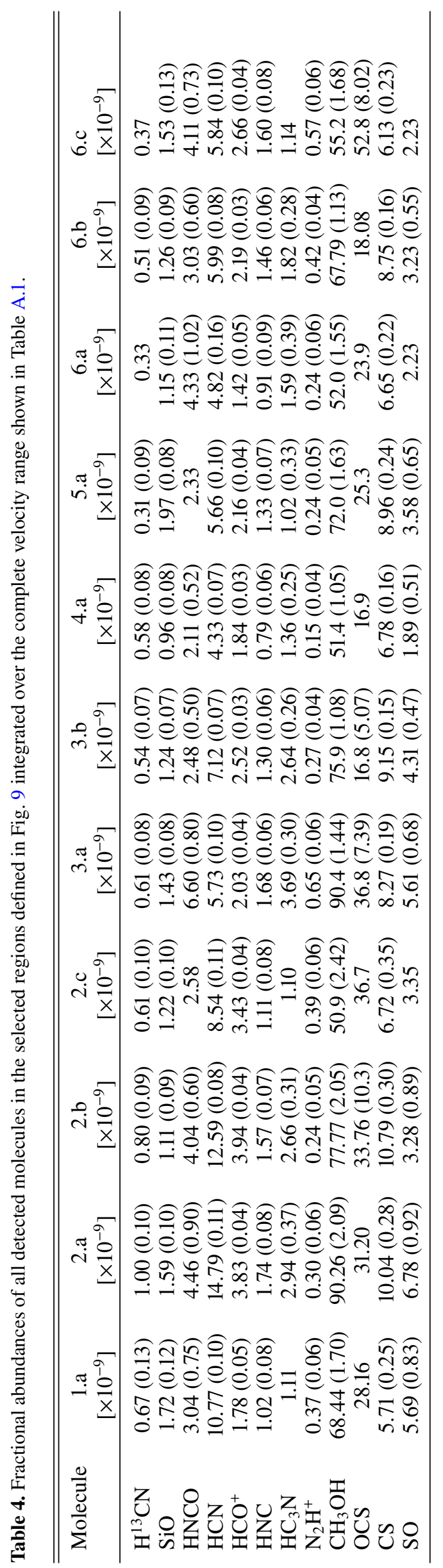

expect that neither X-ray nor cosmic rays produce a significant enhancement of $\mathrm{SiO}$ in this cloud. $\mathrm{SiO}$ has been extensively studied in the GC region, finding high abundance which is associated with large scale shocks (see e.g. Martín-Pintado et al. 1997; Hüttemeister et al. 1998; Menten et al. 2009; Salii et al. 2002; Riquelme et al. 2010b; Minh et al. 2015; Tsuboi et al. 2015, among others). The fractional abundances of $\mathrm{SiO}$ derived in this work are similar to those derived in the $\mathrm{CMZ}$, which is a clear indication that, like in the CMZ, the chemistry in the M-3.8+0.9 molecular cloud is driven by shocks. The highest $\mathrm{SiO}$ abundances are found in positions 1.a, 2.a, 3.a, 3.b and 5.a.

Large abundances of $\mathrm{HNCO}$ and $\mathrm{CH}_{3} \mathrm{OH}$ can also be explained by shocks that release these species from the icy mantles of dust grains. The molecule HNCO is formed efficiently in the solid phase (Hasegawa \& Herbst 1993), and also can be formed by gas-phase reactions (Iglesias 1977). Its abundance is enhanced by grain erosion and disruption by low-velocity shocks $\left(<26 \mathrm{~km} \mathrm{~s}^{-1}\right)$ (Zinchenko et al. 2000) and decreases in the presence of high-velocity shocks $\left(40-50 \mathrm{~km} \mathrm{~s}^{-1}\right)$. Therefore, the shocks that desorb the molecule should be slow enough $\left(<26 \mathrm{~km} \mathrm{~s}^{-1}\right)$ in order do not to dissociate it. In the presence of UV radiation, HNCO is also easily photodissociated, as it is by the UV radiation field induced by shocks (Viti et al. 2002). Martín et al. (2008) performed a systematic study of 13 sources throughout the CMZ and found that this molecule is an excellent discriminator between chemistry driven by shocks and photodissociation. They determined differences up to a factor of 30 in the intensity ratio of $\mathrm{HCNO} /{ }^{13} \mathrm{CS}$ between shielded molecular clouds mostly affected by shocks and those pervaded by intense UV radiation. Using this intensity ratio, they clustered the sources into three groups; typical Galactic center clouds, hot cores, and photon dominated regions (PDRs) and high-velocity shocks, from higher to lower values of this ratio. The fractional abundances of HNCO derived in this work are lower than the ones derived for the typical Galactic center clouds which are affected by shocks, but similar to the hot core sources. It is clear that in the position-position-velocity space occupied by the footpoint of the loops, the UV radiation field is not strong, as indicated by the enhanced HNCO abundances (Fig. C.3). The highest abundances are found in positions 2.a, 2.b, 3.a, and 3.b. As discussed earlier, the HNCO line observed toward position 3.b has a typical shock profile, with a prominent wing, as also seen in the others shock tracer $\left(\mathrm{SiO}, \mathrm{CH}_{3} \mathrm{OH}\right)$. However, here $\mathrm{HNCO}$ is intense only in the peak of the profile, vanishing in the wing, consistent with the idea that this molecule in enhanced in low velocity shocks and is destroyed in higher velocity shocks, as shown by Zinchenko et al. (2000).

Methanol, also ejected from grain mantles by shocks, is another well known shock tracer. Requena-Torres et al. (2006) studied several complex organic molecules, in particular, $\mathrm{CH}_{3} \mathrm{OH}$, in $40 \mathrm{GC}$ molecular clouds. They found high fractional abundances of $\mathrm{CH}_{3} \mathrm{OH}\left(2.4 \times 10^{-8}\right.$ to $\left.1.1 \times 10^{-6}\right)$ similar to those in Table 5 , and estimated that frequent $\left(\sim 10^{5}\right.$ years $)$ shocks with velocities $>6 \mathrm{~km} \mathrm{~s}^{-1}$ are required to explain the high abundances in the gas phase of complex organic molecules in the GC molecular clouds. Jiménez-Serra et al. (2009) show that $\mathrm{CH}_{3} \mathrm{OH}$ show high fractional abundances for low velocity shock $\left(20 \mathrm{~km} \mathrm{~s}^{-1}\right)$ but the abundances are increased for moderate $\left(30 \mathrm{~km} \mathrm{~s}^{-1}\right)$ and high-velocity shock $\left(40 \mathrm{~km} \mathrm{~s}^{-1}\right)$. The methanol line detected in this work corresponds to the $2_{k}-1_{k}$ quartet centered in $96.74 \mathrm{GHz}$, a blend of the $2_{-1}-1-1 E, 2_{0}-1_{0} A^{+}, 2_{0}-$ $1_{0} E, 2_{1}-1_{1} E$ lines, which is considered as one line with the spectroscopic parameters of the most intense one $\left(2_{0}-1_{0} A^{+}\right)$. 
Table 5. Fractional abundances for the CMZ from previous works.

\begin{tabular}{lcccccccc}
\hline \hline Molecule & $\begin{array}{c}1.6 \text { complex }^{a} \\
(20,210)^{e}\end{array}$ & $\begin{array}{c}1.3 \text { complex }^{a} \\
(40,230)^{e}\end{array}$ & $\begin{array}{c}\mathrm{SgrB}^{a} \\
(20,140)^{e}\end{array}$ & $\begin{array}{c}\mathrm{G}_{0.4^{a}} \\
(-20,60)^{e}\end{array}$ & $\begin{array}{c}\mathrm{G} 0.25^{a} \\
(5,100)^{e}\end{array}$ & $\begin{array}{c}50 \mathrm{~km} \mathrm{~s}^{-1 a} \\
(0,100)^{e}\end{array}$ & $\begin{array}{c}20 \mathrm{~km} \mathrm{~s}^{-1 a} \\
(-20,80)^{e}\end{array}$ & $\begin{array}{c}\mathrm{Sgr} \mathrm{C}^{a} \\
(-80,-5)^{e}\end{array}$ \\
\hline & {$\left[\times 10^{-9}\right]$} & {$\left[\times 10^{-9}\right]$} & {$\left[\times 10^{-9}\right]$} & {$\left[\times 10^{-9}\right]$} & {$\left[\times 10^{-9}\right]$} & {$\left[\times 10^{-9}\right]$} & {$\left[\times 10^{-9}\right]$} & {$\left[\times 10^{-9}\right]$} \\
\hline $\mathrm{H}^{13} \mathrm{CN}$ & $1.11(0.06)$ & $1.34(0.03)$ & $0.66(0.02)$ & $1.02(0.03)$ & $1.17(0.02)$ & $1.63(0.01)$ & $1.53(0.02)$ & $0.61(0.03)$ \\
$\mathrm{SiO}$ & $2.47(0.08)$ & $2.60(0.04)$ & $1.12(0.02)$ & $1.40(0.03)$ & $1.05(0.02)$ & $1.40(0.01)$ & $1.60(0.03)$ & $0.50(0.05)$ \\
$\mathrm{HNCO}$ & $41.13(0.40)$ & $10.39(0.23)$ & $26.32(0.17)$ & $21.12(0.19)$ & $14.32(0.17)$ & $10.52(0.09)$ & $17.62(0.23)$ & $3.62(0.26)$ \\
$\mathrm{HCN}$ & $14.92(0.05)$ & $17.18(0.08)$ & $>10.34$ & $13.27(0.03)$ & $15.95(0.02)$ & $>23.19$ & $22.30(0.03)$ & $8.22(0.05)$ \\
$\mathrm{HCO}+$ & $4.17(0.04)$ & $5.17(0.03)$ & $>3.96$ & $2.25(0.01)$ & $2.74(0.02)$ & $3.50(0.01)$ & $4.60(0.01)$ & $1.87(0.02)$ \\
$\mathrm{HNC}$ & $5.14(0.05)$ & $2.43(0.02)$ & $>3.48$ & $8.37(0.02)$ & $6.93(0.02)$ & $3.95(0.01)$ & $8.20(0.02)$ & $2.02(0.02)$ \\
$\mathrm{HC}_{3} \mathrm{~N}$ & $7.84(0.22)$ & $4.71(0.13)$ & $12.41(0.09)$ & $5.16(0.08)$ & $7.00(0.07)$ & $7.46(0.04)$ & $8.45(0.06)$ & $1.63(0.11)$ \\
$\mathrm{N}_{2} \mathrm{H}^{+}$ & $1.85(0.04)$ & $0.88(0.02)$ & $1.39(0.01)$ & $2.30(0.02)$ & $2.28(0.01)$ & $1.48(0.01)$ & $2.58(0.01)$ & $1.03(0.01)$ \\
$\mathrm{CH}{ }_{3} \mathrm{OH}$ & & & & & & & & \\
$\mathrm{OCS}$ & & & & & & & & \\
$\mathrm{CS}$ & $9.34(0.00)$ & $18.60(1.77)$ & $32.40(1.60)$ & $12.43(1.04)$ & $10.69(0.92)$ & $23.37(0.58)$ & $23.27(1.06)$ & $15.39(1.49)$ \\
$\mathrm{SO}$ & & & & & & & & \\
\hline
\end{tabular}

\begin{tabular}{|c|c|c|c|c|c|}
\hline Molecule & $+0.693^{c}$ & $-0.11^{c}$ & $\operatorname{Sgr} \mathrm{B} 2 \mathrm{~N}^{d, f}$ & $\operatorname{Sgr} \mathrm{B} 2 \mathrm{M}^{d, f}$ & $\begin{array}{c}\mathrm{CND}^{g} \\
(-30,-30)\end{array}$ \\
\hline & {$\left[\times 10^{-9}\right]$} & {$\left[\times 10^{-9}\right]$} & {$\left[\times 10^{-9}\right]$} & {$\left[\times 10^{-9}\right]$} & {$\left[\times 10^{-9}\right]$} \\
\hline $\mathrm{H}^{13} \mathrm{CN}$ & $>6$ & $>9$ & 8.72 & 1.30 & 3.48 \\
\hline $\mathrm{SiO}$ & $1.4(0.2)$ & $7.2(0.2)$ & 0.49 & 0.65 & 2.85 \\
\hline $\mathrm{HNCO}$ & 24.7 (1.4) & $28.3(3.0)$ & 2.78 & 85.07 & \\
\hline $\mathrm{HCN}$ & $>12$ & $>180$ & 173.70 & 25.94 & 121.25 \\
\hline $\mathrm{HCO}^{+}$ & $9.6(2.2)$ & 15.5 & 8.23 & 10.80 & 18.87 \\
\hline $\mathrm{HNC}$ & $>42$ & $>60$ & 7.78 & 8.67 & 6.82 \\
\hline $\mathrm{HC}_{3} \mathrm{~N}$ & $9.4(1.0)$ & $11.2(1.0)$ & 19.48 & 0.65 & 1.03 \\
\hline $\mathrm{N}_{2} \mathrm{H}^{+}$ & & 0.81 & 1.42 & 0.53 & \\
\hline $\mathrm{CH}_{3} \mathrm{OH}$ & 2160 (257) & $<1670$ & 28571.43 & 23.62 & \\
\hline OCS & $31.8(1.5)$ & $36.4(6.5)$ & 292.21 & 4.06 & \\
\hline CS & $12.43(1.04)$ & & 420.45 & 21.59 & 38.38 \\
\hline SO & $10.4(8.8)$ & $7.8(2.0)$ & 1316.56 & 13.01 & 16.0 \\
\hline
\end{tabular}

Notes. ${ }^{(a)}$ Jones et al. (2012), ${ }^{(b)}$ we used the ${ }^{13} \mathrm{CS}$ emission and a ${ }^{12} \mathrm{C} /{ }^{13} \mathrm{C}$ ratio of 24 to derive the fractional abundance of ${ }^{12} \mathrm{CS}$, The column densities of $\mathrm{HCN}, \mathrm{HCO}^{+}$and $\mathrm{HNC}$ were corrected by opacity following the formulation in Jones et al. (2012). In SgrB, the ${ }^{13} \mathrm{C}$ isotopic substitution could be affected by opacity as shown in Fig. A.6, therefore the fractional abundances are a lower limits. The same is true in the $50 \mathrm{~km} \mathrm{~s}{ }^{-1}$ cloud for $\mathrm{H}^{13} \mathrm{CN}$. ${ }^{(c)}$ Armijos-Abendaño et al. (2015), ${ }^{(d)}$ Belloche et al. (2013), ${ }^{(e)}$ integrate velocity range $\left[\mathrm{km} \mathrm{s}^{-1}\right] .{ }^{(f)}$ Fractional abundance of the velocity component closest to the nominal velocity of $\mathrm{Sgr} B 2 \mathrm{~N}$ and $\mathrm{Sgr} \mathrm{B} 2 \mathrm{M}$. The $\mathrm{N}\left(\mathrm{H}_{2}\right)$ value was derived from $\mathrm{N}\left({ }^{13} \mathrm{CO}\right)$ with the conversion factor used for the CMZ in this work. ${ }^{(g)}$ Harada et al. (2015). The $\mathrm{H}_{2}$ column density was estimated from multi-transition LVG analysis of CO using an abundance ratio $\mathrm{CO} / \mathrm{H}_{2}=8 \times 10^{-5}$.

Menten et al. (2009) observed this line and also SiO and CS towards a molecular cloud affected by shocks at the edge of the $\mathrm{CMZ}$ and found that this methanol line was the most intense.

In our work, the $\mathrm{CH}_{3} \mathrm{OH}$ emission is one of the most intense emission line (after those of $\mathrm{HCN}$ ), with the largest fractional abundances in positions 2.a, 2.b, 3.a, and 5.a. This, the large terminal velocity found in position 3.b (which indicates high velocity shock), and the similar abundances of $\mathrm{SiO}$ in the M-3.8+0.9 molecular cloud and in the CMZ, indicate that the chemistry in the M-3.8+0.9 molecular cloud may be driven by moderate or high velocity shocks.

\subsection{High temperature gas}

Amo-Baladrón et al. (2011) suggest a correlation between the abundance ratio $\mathrm{HNC} / \mathrm{HCN}$ and temperature, with values close to one in quiescent cool dark clouds, a decrease by between one and two orders of magnitudes in the warmer giant molecular clouds near sites of massive star formation, and with values ranging between 0.013-0.2 near the PDR in the giant molecular cloud OMC-1, and in the immediate vicinity of the hot core Orion-KL (Schilke et al. 1992). The decrease of this ratio is due to destruction processes of HNC produced by neutral-neutral reactions with an activation barrier (Pineau des Fôrets et al. 1990) higher than $190 \mathrm{~K}$ (Hirota et al. 1998) or $300 \mathrm{~K}$ (Talbi et al. 1996). From our PCA analysis, Fig. 8 shows that the HCN and HNC molecular emission appears to be anti-correlated but only at $2 \%$ variance, in contrast with higher values found in other places in the Galaxy (Lo et al. 2009). Figure 7 shows the spatial distribution of this anti-correlation, which indicates that in positions 1.a, 2.a, 2.b, and 2.c the HNC abundance decreases, suggesting that those regions should be the warmer regions in the $M-3.8+0.9$ 
molecular cloud. This is consistent with the kinetic temperatures derived by Torii et al. (2010a) who find their highest value in our position 2.b. From Table A.1 we can see that the HNC/HCN abundance ratio ranges from 0.1 to 0.15 in those regions (1.a to 2.c).

\subsection{Comparison with previous work on the footpoints}

Our results indicate that the chemistry of the $\mathrm{M}-3.8+0.9$ molecular cloud is mainly driven by shocks. As discussed in Sect. 1, Fukui et al. (2006) proposed that the GMLs are formed by a magnetic buoyancy caused by a Parker instability, and they argue that the footpoint of these loops, which are two bright spots at both ends, are formed by the accumulation of gas that flows down along the loops. If the GMLs scenario applies, two footpoints should coexist in the $\mathrm{M}-3.8+0.9$ cloud, the western side of the loop 1 and the eastern side of the loop 2. These footpoints were studied by Torii et al. (2010a) and Kudo et al. (2011) using multi-transition observations of $\mathrm{CO}$ and ${ }^{13} \mathrm{CO}$. They found several features in the M-3.8+0.9 molecular cloud, in particular two U-shapes in the longitude-latitude and latitude-velocity space, which they explain as a consequence of the magnetic loops as predicted by magnetohydrodynamical (MHD) simulations (Takahashi et al. 2009). These features are also seen in the molecular emission presented in this work. Torii et al. (2010a) also found an inverted-triangle feature that they called the protrusion, which corresponds to our Complex 6. Complexes 3 and 6 both show a sharp intensity gradient studied by Torii et al. (2010a) and both mark the eastern side of the U-shape, which is an indication that they share similar physical properties. They also identified three additional broad velocity features, which we identified as the positions 1.a, 2.b, and 5.a in Fig. 7. They connect both sides of the U-shapes which are not yet explained by the MHD models.

\section{Conclusions}

We have mapped the $\mathrm{M}-3.8+0.9$ molecular cloud in various 3-mm molecular lines using the 22-m Mopra telescope, and the $J=2-1$ rotational transition of ${ }^{13} \mathrm{CO}$ using 12-m APEX telescope. Eleven molecular species were detected from the 3 -mm survey and the ${ }^{13} \mathrm{C}$ isotopic substitution of $\mathrm{HCN}$. These molecules encompass tracers of different physical processes, such as shock tracer $\left(\mathrm{SiO}, \mathrm{HNCO}\right.$, and $\left.\mathrm{CH}_{3} \mathrm{OH}\right)$, medium and high density tracers $\left(\mathrm{CS}, \mathrm{HCN}, \mathrm{HNC}, \mathrm{HCO}^{+}\right.$, and $\mathrm{N}_{2} \mathrm{H}^{+}$), and one with a high photodissociation rate (HNCO).

The molecular cloud shows a velocity gradient from higher to lower velocities from the north-western to the south-eastern direction. Both the longitude-latitude and the latitude-velocity plots show the U-shapes observed in the $\mathrm{CO}$ emission in previous work which supports the GMLs phenomenon. We identified six molecular complexes throughout the molecular cloud. We performed a PCA analysis using the six most intense and widespread lines. Based on it, 11 positions were selected that show higher or lower correlations between species. We derived the fractional abundances for these positions for all detected molecules. The ${ }^{13} \mathrm{CO}$ emission was used as a tracer of the molecular hydrogen and to derive its column density, $\mathrm{N}\left(\mathrm{H}_{2}\right)$. The fractional abundances in the footpoint of the GMLs were compared with the fractional abundances in the CMZ, and we found that $\mathrm{SiO}$ abundance in the CMZ and GMLs are similar. Based on the high abundance of shock tracers in the $\mathrm{M}-3.8+0.9$ molecular cloud, in particular $\mathrm{SiO}$ and the moderate abundance of $\mathrm{HNCO}$ and $\mathrm{CH}_{3} \mathrm{OH}$, we conclude that moderate $\left(30 \mathrm{~km} \mathrm{~s}^{-1}\right)$ or even high velocity shocks (40-50 $\mathrm{km} \mathrm{s}^{-1}$ ) are the dominant physical process heating and driving the chemistry of the molecular gas in the $\mathrm{M}-3.8+0.9$ molecular cloud.

Acknowledgements. D.R. acknowledges fruitful discussions with colleagues from Nagoya University, especially with Yasuo Fukui, Kazufumi Torii and Rei Enokiya. We thank to Izaskun Jimenez-Serra and Esteban F.E. Morales for useful discussions. This work was partially carried out within the Collaborative Research Council 956, subproject A5, funded by the Deutsche Forschungsgemeinschaft (DFG). D.R. was supported by DGI grant AYA 200806181-C02-02 during the observations. LB acknowledges support by CONICYT grant PFB-06. JM-P acknowledges partial support by the MINECO under grants AYA2010-2169-C04-01, FIS2012-39162-C06-01, ESP2013-47809-C03-01 and ESP2015-65597-C4-1. We thank the anonymous referee for critical reading and constructive comments that helped to improve this manuscript. The Mopra radio telescope is part of the Australia Telescope National Facility which is funded by the Australian Government for operation as a National Facility managed by CSIRO. The University of New South Wales Digital Filter Bank used for the observations with the Mopra Telescope was provided with support from the Australian Research Council. This publication is based on data acquired with the Atacama Pathfinder Experiment (APEX). APEX is a collaboration between the Max-Planck-Institut fur Radioastronomie, the European Southern Observatory, and the Onsala Space Observatory. This research made use of Astropy, a community-developed core Python package for Astronomy (Astropy Collaboration et al. 2013).

\section{References}

Amo-Baladrón, M. A., Martín-Pintado, J., Morris, M. R., Muno, M. P., \& Rodríguez-Fernández, N. J. 2009, ApJ, 694, 943

Amo-Baladrón, M. A., Martín-Pintado, J., \& Martín, S. 2011, A\&A, 526, A54

Ao, Y., Henkel, C., Menten, K. M., et al. 2013, A\&A, 550, A135

Armijos-Abendaño, J., Martín-Pintado, J., Requena-Torres, M. A., Martín, S., \& Rodríguez-Franco, A. 2015, MNRAS, 446, 3842

Astropy Collaboration, Robitaille, T. P., Tollerud, E. J., et al. 2013, A\&A, 558, A33

Bania, T. M. 1980, ApJ, 242, 95

Bania, T. M., Stark, A. A., \& Heiligman, G. M. 1986, ApJ, 307, 350

Belloche, A., Müller, H. S. P., Menten, K. M., Schilke, P., \& Comito, C. 2013, A\&A, 559, A47

Binney, J., Gerhard, O. E., Stark, A. A., Bally, J., \& Uchida, K. I. 1991, MNRAS, 252, 210

Bitran, M., Alvarez, H., Bronfman, L., May, J., \& Thaddeus, P. 1997, A\&AS, 125,99

Bland-Hawthorn, J., \& Cohen, M. 2003, ApJ, 582, 246

Brogan, C. L., Nord, M., Kassim, N., Lazio, J., \& Anantharamaiah, K. 2003, Astron. Nachr. Suppl., 324, 17

Caswell, J. L., \& Haynes, R. F. 1987, A\&A, 171, 261

Dahmen, G., Hüttemeister, S., Wilson, T. L., \& Mauersberger, R. 1998, A\&A, 331,959

Dame, T. M., \& Thaddeus, P. 2008, ApJ, 683, L143

Farquhar, P. R. A., Millar, T. J., \& Herbst, E. 1994, MNRAS, 269, 641

Frerking, M. A., Langer, W. D., \& Wilson, R. W. 1982, ApJ, 262, 590

Fujishita, M., Torii, K., Kudo, N., et al. 2009, PASJ, 61, 1039

Fukui, Y., Yamamoto, H., Fujishita, M., et al. 2006, Science, 314, 106

Garwood, R. W. 2000, in Astronomical Data Analysis Software and Systems IX, eds. N. Manset, C. Veillet, \& D. Crabtree, ASP Conf. Ser., 216, 243

Ginsburg, A., Henkel, C., Ao, Y., et al. 2016, A\&A, 586, A50

Goldsmith, P. F., \& Langer, W. D. 1999, ApJ, 517, 209

Güsten, R., Nyman, L. A., Schilke, P., et al. 2006, A\&A, 454, L13

Harada, N., Riquelme, D., Viti, S., et al. 2015, A\&A, 584, A102

Hasegawa, T. I., \& Herbst, E. 1993, MNRAS, 263, 589

Heyer, M. H., \& Schloerb, F. 1997, ApJ, 475, 173

Hirota, T., Yamamoto, S., Mikami, H., \& Ohishi, M. 1998, ApJ, 503, 717

Hüttemeister, S., Dahmen, G., Mauersberger, R., et al. 1998, A\&A, 334, 646

Iglesias, E. 1977, ApJ, 218, 697

Jackson, J. M., Armstrong, J. T., \& Barrett, A. H. 1984, ApJ, 280, 608

Jiménez-Serra, I., Martín-Pintado, J., Caselli, P., Viti, S., \& Rodríguez-Franco, A. $2009, \mathrm{ApJ}, 695,149$

Jones, P. A., Burton, M. G., Cunningham, M. R., et al. 2008, MNRAS, 386, 117 Jones, P. A., Burton, M. G., Cunningham, M. R., et al. 2012, MNRAS, 419, 2961 Jones, P. A., Burton, M. G., Cunningham, M. R., Tothill, N. F. H., \& Walsh, A. J. 2013, MNRAS, 433, 221 
Kaneda, H., Ishihara, D., Mouri, A., et al. 2012, PASJ, 64, 25

Klein, B., Hochgürtel, S., Krämer, I., et al. 2012, A\&A, 542, L3

Kudo, N., Torii, K., Machida, M., et al. 2011, PASJ, 63, 171

Ladd, N., Purcell, C., Wong, T., \& Robertson, S. 2005, Publ. Astron. Soc. Aust., 22,62

Langer, W. D., \& Penzias, A. A. 1990, ApJ, 357, 477

Lo, N., Cunningham, M. R., Jones, P. A., et al. 2009, MNRAS, 395, 1021

Machida, M., Matsumoto, R., Nozawak, S., et al. 2009, PASJ, 61, 411

Mangum, J. G., Emerson, D. T., \& Greisen, E. W. 2007, A\&A, 474, 679

Martín, S., Requena-Torres, M. A., Martín-Pintado, J., \& Mauersberger, R. 2008, ApJ, 678, 245

Martín-Pintado, J., Bachiller, R., \& Fuente, A. 1992, A\&A, 254, 315

Martín-Pintado, J., de Vicente, P., Fuente, A., \& Planesas, P. 1997, ApJ, 482, L45

Martín-Pintado, J., de Vicente, P., Rodríguez-Fernández, N. J., Fuente, A., \& Planesas, P. 2000, A\&A, 356, L5

Mauersberger, R., \& Henkel, C. 1989, A\&A, 223, 79

Menten, K. M., Wilson, R. W., Leurini, S., \& Schilke, P. 2009, ApJ, 692, 47

Minh, Y. C., Liu, H. B., Su, Y.-N., et al. 2015, ApJ, 808, 86

Morris, M., \& Serabyn, E. 1996, ARA\&A, 34, 645

Müller, H. S. P., Thorwirth, S., Roth, D. A., \& Winnewisser, G. 2001, A\&A 370, L49

Müller, H. S. P., Schlöder, F., Stutzki, J., \& Winnewisser, G. 2005, J. Mol. Struct., 742,215

Pickett, H. M., Poynter, R. L., Cohen, E. A., et al. 1998, J. Quant. Spectr. Rad. Transf., 60, 883

Pineau des Fôrets, G., Roueff, E., \& Flower, D. R. 1990, MNRAS, 244, 668
Requena-Torres, M. A., Martín-Pintado, J., Rodríguez-Franco, A., et al. 2006, A\&A, 455, 971

Riquelme, D., Amo-Baladrón, M. A., Martín-Pintado, J., et al. 2010a, A\&A, 523, A51

Riquelme, D., Bronfman, L., Mauersberger, R., May, J., \& Wilson, T. L. 2010b, A\&A, 523, A45

Riquelme, D., Amo-Baladrón, M. A., Martín-Pintado, J., et al. 2013, A\&A, 549, A36

Roberts, M. S. E., Romani, R. W., \& Kawai, N. 2001, ApJS, 133, 451

Rodríguez-Fernández, N. J., Martín-Pintado, J., Fuente, A., et al. 2001, A\&A, 365, 174

Salii, S. V., Sobolev, A. M., \& Kalinina, N. D. 2002, Astron. Rep., 46, 955

Schilke, P., Walmsley, C. M., Pineau Des Fôrets, G., et al. 1992, A\&A, 256,595

Shlens, J. 2014, ArXiv e-prints [arXiv: 1404.1100]

Takahashi, K., Nozawa, S., Matsumoto, R., et al. 2009, PASJ, 61, 957

Talbi, D., Ellinger, Y., \& Herbst, E. 1996, A\&A, 314, 688

Torii, K., Kudo, N., Fujishita, M., et al. 2010a, PASJ, 62, 675

Torii, K., Kudo, N., Fujishita, M., et al. 2010b, PASJ, 62, 1307

Tsuboi, M., Miyazaki, A., \& Uehara, K. 2015, PASJ, 67, 90

Ungerechts, H., Bergin, E. A., Goldsmith, P. F., et al. 1997, ApJ, 482, 245

Vassilev, V., Meledin, D., Lapkin, I., et al. 2008, A\&A, 490, 1157

Viti, S., Natarajan, S., \& Williams, D. A. 2002, MNRAS, 336, 797

Wilson, T. L., \& Matteucci, F. 1992, A\&ARv, 4, 1

Wilson, T. L., \& Rood, R. 1994, ARA\&A, 32, 191

Yusef-Zadeh, F., Wardle, M., Lis, D., et al. 2013, J. Phys. Chem. A, 117, 9404

Zinchenko, I., Henkel, C., \& Mao, R. Q. 2000, A\&A, 361, 1079 
Appendix A: Complementary tables and figures

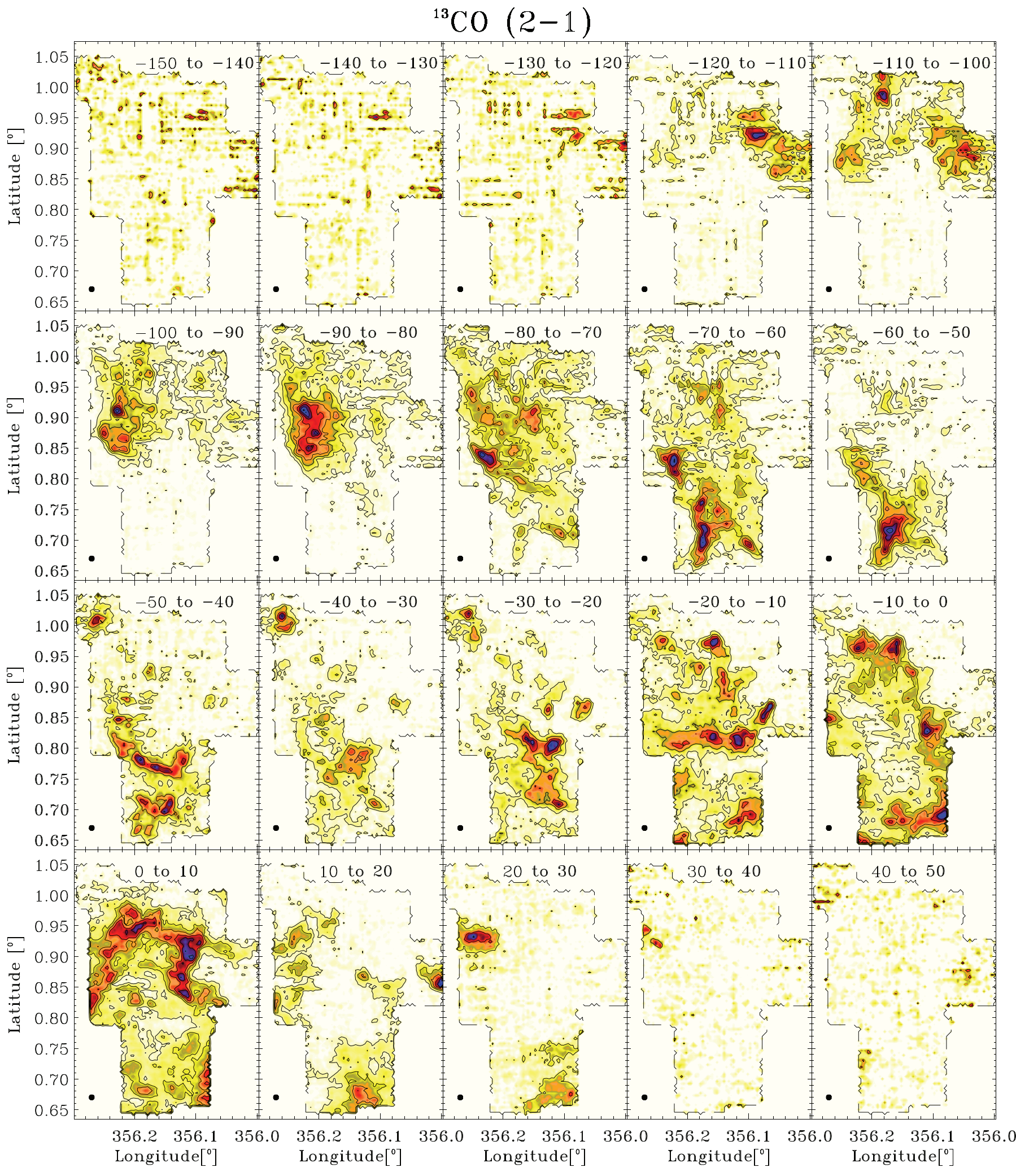

$\left[\mathrm{K} \mathrm{km} \mathrm{s}^{-1}\right]$

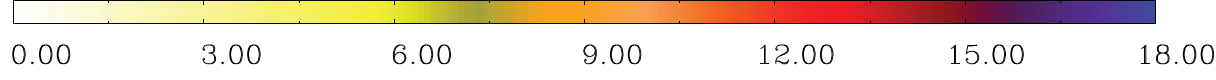

Fig. A.1. Integrated brightness temperature of the $\mathrm{M}-3.8+0.9$ molecular cloud in ${ }^{13} \mathrm{CO}(2-1)$ in velocity intervals of $10 \mathrm{~km} \mathrm{~s}$. 


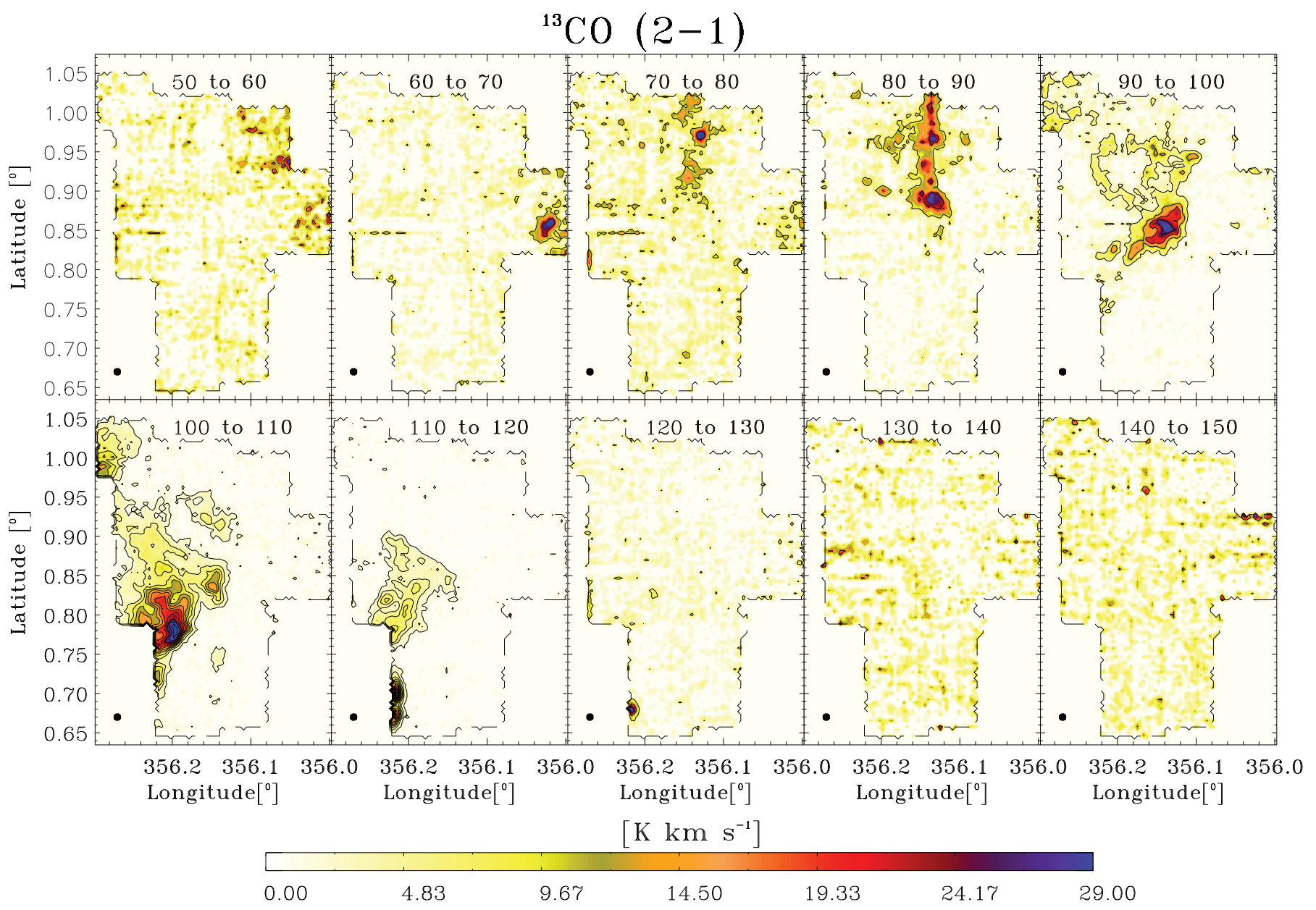

Fig. A.2. Integrated brightness temperature of the $\mathrm{M}-3.8+0.9$ molecular cloud in ${ }^{13} \mathrm{CO}(2-1)$ in velocity intervals of $10 \mathrm{~km} \mathrm{~s}{ }^{-1}$, continuation.

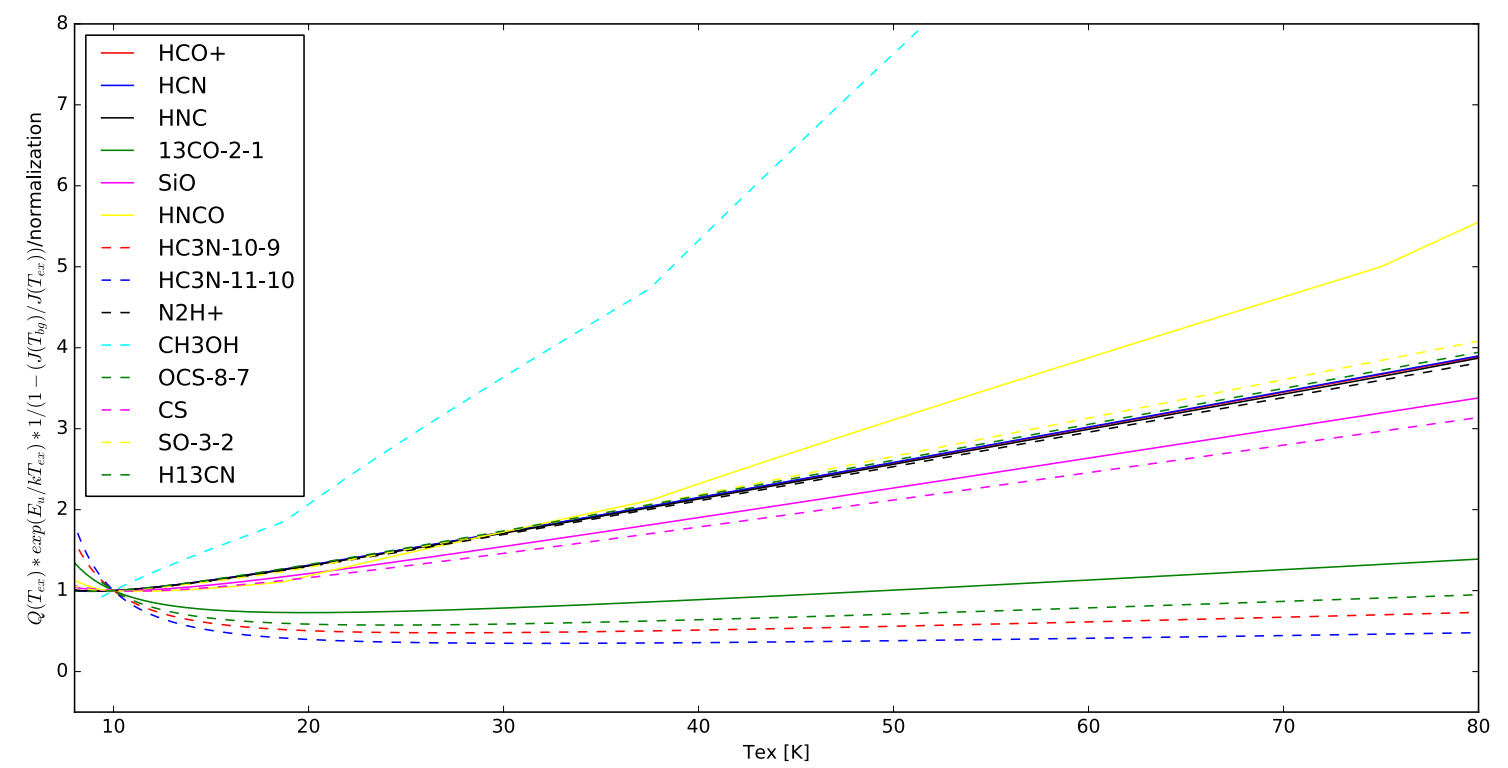

Fig. A.3. Variation of column density with excitation temperature, $T_{\mathrm{ex}}$. The $y$-axis shows the $T_{\mathrm{ex}}$-dependent parameters in Eq. (1) normalized to $T_{\mathrm{ex}}=10 \mathrm{~K}$. For ${ }^{13} \mathrm{CO}(2-1)$, we can see that the difference between the assumption of $T_{\mathrm{ex}}=10 \mathrm{~K}$ and $40 \mathrm{~K}$ (which is the temperature estimated by the detailed work of Torii et al. (2010a) is $\sim 10 \%$. Most of the species in the 3-mm molecular transitions show a factor of less than two if we increase the $T_{\text {ex }}$ at $40 \mathrm{~K}$, with the exception of $\mathrm{CH}_{3} \mathrm{OH}$ which is highly dependent of the $T_{\text {ex }}$; and $\mathrm{OCS}$ and $\mathrm{HC}_{3} \mathrm{~N}$. 
CMZ HCN

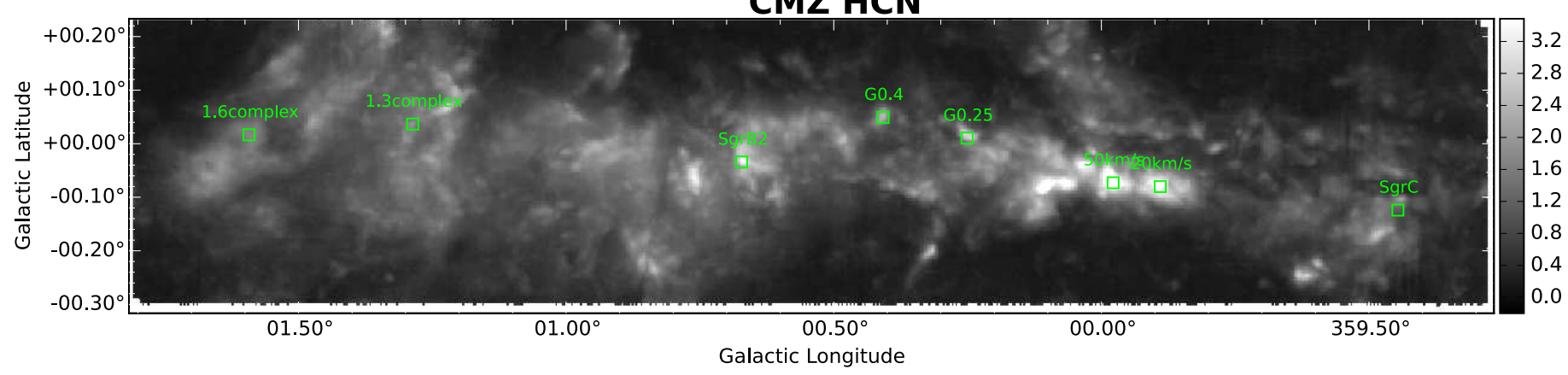

Fig. A.4. Selected regions for deriving the column densities for the detected molecules of this work overlaid on the peak brightness images of HCN from Jones et al. (2012)

Table A.1. Column density of the detected molecules.

\begin{tabular}{|c|c|c|c|c|c|c|c|c|}
\hline & $\begin{array}{c}\text { Velocity range } \\
{\left[\mathrm{km} \mathrm{s}^{-1}\right]}\end{array}$ & $\begin{array}{c}\mathrm{H}^{13} \mathrm{CN} \\
{\left[\times 10^{13} \mathrm{~cm}^{-2}\right]}\end{array}$ & $\begin{array}{c}\mathrm{SiO} \\
\left.\times 10^{13} \mathrm{~cm}^{-2}\right] \\
\end{array}$ & $\begin{array}{c}\mathrm{HNCO} \\
{\left[\times 10^{13} \mathrm{~cm}^{-2}\right]}\end{array}$ & $\begin{array}{c}\mathrm{HCN} \\
{\left[\times 10^{13} \mathrm{~cm}^{-2}\right]}\end{array}$ & $\begin{array}{c}\mathrm{HCO}^{+} \\
{\left[\times 10^{13} \mathrm{~cm}^{-2}\right]}\end{array}$ & $\begin{array}{c}\mathrm{HNC} \\
{\left[\times 10^{13} \mathrm{~cm}^{-2}\right]}\end{array}$ & $\begin{array}{c}\mathrm{HC}_{3} \mathrm{~N} \\
{\left[\times 10^{13} \mathrm{~cm}^{-2}\right]}\end{array}$ \\
\hline $\begin{array}{l}1 . \mathrm{a} \\
2 . \mathrm{a} \\
2 . \mathrm{b} \\
2 . \mathrm{c} \\
3 . \mathrm{a} \\
3 . \mathrm{b} \\
4 . \mathrm{a} \\
5 . \mathrm{a} \\
6 . \mathrm{a} \\
6 . \mathrm{b} \\
6 . \mathrm{c}\end{array}$ & $\begin{array}{l}0-20 \\
0-20 \\
o-20 \\
o-20 \\
o-50 \\
o-20 \\
o-20 \\
o-20 \\
o-20 \\
-30 \\
-20\end{array}$ & $\begin{array}{c}0.66 \pm 0.07 \\
0.44 \pm 0.07 \\
0.55 \pm 0.07 \\
0.65 \pm 0.09 \\
0.55 \pm 0.07 \\
0.21 \pm 0.06 \\
0.25 \\
0.39 \pm 0.07 \\
0.20\end{array}$ & $\begin{array}{l}7 \\
7 \\
7 \\
8 \\
7 \\
6 \\
6 \\
8 \\
7 \\
7\end{array}$ & $\begin{array}{r}3.34 \pm \\
1.8 \\
5.95 \pm \\
2.96 \pm \\
2.02 \pm \\
1.5 \\
3.32 \pm \\
2.35 \pm \\
2.20 \pm\end{array}$ & $\begin{array}{c}4.71 \pm 0.05 \\
8.96 \pm 0.07 \\
10.39 \pm 0.06 \\
6.12 \pm 0.08 \\
5.17 \pm 0.09 \\
8.51 \pm 0.08 \\
4.15 \pm 0.07 \\
3.87 \pm 0.07 \\
3.69 \pm 0.12 \\
4.63 \pm 0.06 \\
3.12 \pm 0.05\end{array}$ & $\begin{array}{l}3 \\
3 \\
4 \\
3 \\
2\end{array}$ & $\begin{array}{l}5 \\
6 \\
6 \\
7 \\
6 \\
5 \\
7 \\
5 \\
4\end{array}$ & $\begin{array}{c}1.78 \pm 0.22 \\
2.19 \pm 0.26 \\
0.79 \\
3.32 \pm 0.27 \\
3.16 \pm 0.31 \\
1.30 \pm 0.24 \\
0.70 \pm 0.22 \\
1.22 \pm 0.30 \\
1.40 \pm 0.21 \\
0.61\end{array}$ \\
\hline & $\begin{array}{c}\text { Velocity range } \\
{\left[\mathrm{km} \mathrm{s}^{-1}\right]}\end{array}$ & $\begin{array}{c}\mathrm{N}_{2} \mathrm{H}^{+} \\
{\left[\times 10^{13} \mathrm{~cm}^{-2}\right]}\end{array}$ & $\begin{array}{c}\mathrm{CH}_{3} \mathrm{OH} \\
{\left[\times 10^{13} \mathrm{~cm}^{-2}\right]}\end{array}$ & $\begin{array}{c}\text { OCS } \\
{\left[\times 10^{13} \mathrm{~cm}^{-2}\right]}\end{array}$ & $\begin{array}{c}\mathrm{CS} \\
{\left[\times 10^{13} \mathrm{~cm}^{-2}\right]}\end{array}$ & $\begin{array}{c}\mathrm{SO} \\
{\left[\times 10^{13} \mathrm{~cm}^{-2}\right]}\end{array}$ & $\begin{array}{c}{ }^{13} \mathrm{CO} \\
{\left[\times 10^{16} \mathrm{~cm}^{-2}\right]}\end{array}$ & $\begin{array}{c}\mathrm{H}_{2} \\
{\left[\times 10^{21} \mathrm{~cm}^{-2}\right]}\end{array}$ \\
\hline $\begin{array}{l}1 . \mathrm{a} \\
2 . \mathrm{a} \\
2 . \mathrm{b} \\
2 . \mathrm{c} \\
\text { 3.a } \\
3 . \mathrm{b} \\
4 . \mathrm{a} \\
5 . \mathrm{a} \\
6 . \mathrm{a} \\
6 . \mathrm{b} \\
6 . \mathrm{c}\end{array}$ & $\begin{array}{l}-90 \text { to }-20 \\
-120 \text { to }-20 \\
-120 \text { to }-20 \\
-120 \text { to }-20 \\
-120 \text { to }-50 \\
-120 \text { to }-20 \\
-100 \text { to }-20 \\
-100 \text { to }-20 \\
-85 \text { to }-20 \\
-85 \text { to }-30 \\
-80 \text { to }-20\end{array}$ & $\begin{array}{l}0.16 \pm 0.03 \\
0.18 \pm 0.03 \\
0.20 \pm 0.04 \\
0.28 \pm 0.04 \\
0.59 \pm 0.05 \\
0.32 \pm 0.05 \\
0.14 \pm 0.04 \\
0.17 \pm 0.04 \\
0.18 \pm 0.05 \\
0.32 \pm 0.03 \\
0.30 \pm 0.03\end{array}$ & $\begin{array}{l}29.94 \pm 0.74 \\
54.68 \pm 1.26 \\
64.17 \pm 1.69 \\
36.51 \pm 1.73 \\
81.52 \pm 1.29 \\
90.86 \pm 1.29 \\
49.31 \pm 1.01 \\
49.19 \pm 1.11 \\
39.86 \pm 1.19 \\
52.39 \pm 0.87 \\
29.53 \pm 0.90\end{array}$ & $\begin{array}{c}20.16 \pm 6.06 \\
16.19 \\
17.31 \\
18.34 \\
13.97 \\
28.26 \pm 4.29\end{array}$ & $\begin{array}{c}8.90 \pm 0.24 \\
4.81 \pm 0.25 \\
7.46 \pm 0.17 \\
10.93 \pm 0.18 \\
6.50 \pm 0.15 \\
6.12 \pm 0.16 \\
5.09 \pm 0.17 \\
6.76 \pm 0.12 \\
3.28 \pm 0.12\end{array}$ & $\begin{array}{c}2.49 \pm 0.36 \\
4.11 \pm 0.56 \\
2.71 \pm 0.74 \\
2.40\end{array}$ & $\begin{array}{l}0.83 \pm 0.02 \\
1.15 \pm 0.03 \\
1.57 \pm 0.02 \\
1.36 \pm 0.04 \\
1.71 \pm 0.02 \\
2.27 \pm 0.03 \\
1.82 \pm 0.02 \\
1.30 \pm 0.02 \\
1.46 \pm 0.02 \\
1.47 \pm 0.02 \\
1.02 \pm 0.02\end{array}$ & $\begin{array}{c}4.37 \pm 0.12 \\
6.06 \pm 0.14 \\
8.25 \pm 0.12 \\
7.16 \pm 0.21 \\
9.01 \pm 0.10 \\
11.96 \pm 0.13 \\
9.58 \pm 0.10 \\
6.83 \pm 0.09 \\
7.66 \pm 0.11 \\
7.73 \pm 0.09 \\
5.35 \pm 0.09\end{array}$ \\
\hline
\end{tabular}

Notes. ${ }^{(a)}$ Column density obtained from $\mathrm{HC}_{3} \mathrm{~N}(10-9)$ because this transition has higher signal-to-noise ratio than the (11-10).

(b) $\mathrm{N}\left(\mathrm{H}_{2}\right)$ derived from ${ }^{13} \mathrm{CO}$ emission using a conversion factor of $1.9 \times 10^{-6}$ (see text for details) 
D. Riquelme et al.: Giant molecular loops in the GC region
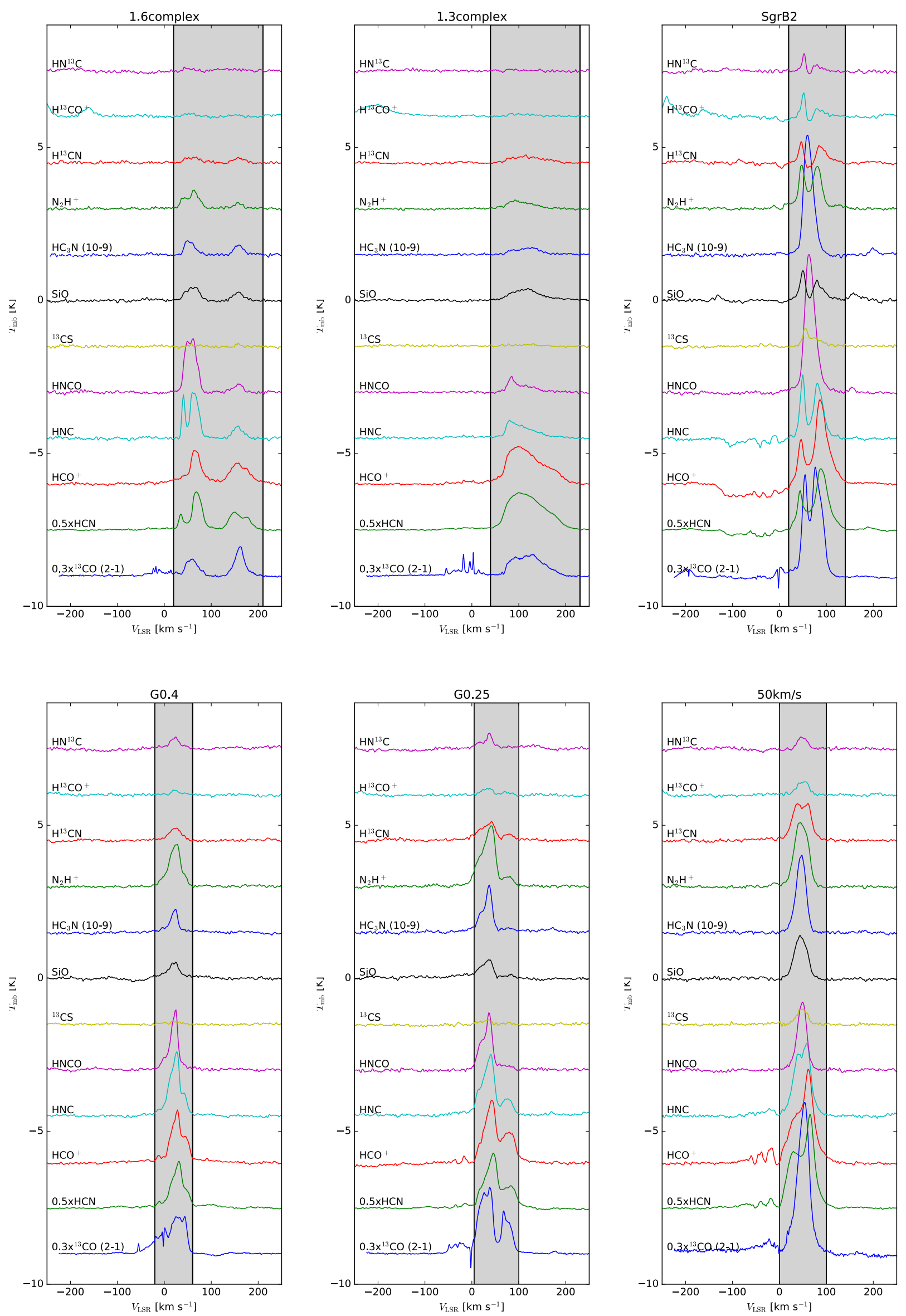

Fig. A.5. Average spectra for each region shown in Fig. A.4. ${ }^{13} \mathrm{CO}(2-1)$ spectra are scaled by a factor of 0.3 and $\mathrm{HCN}$ spectra are scaled by a factor of 0.5 . 

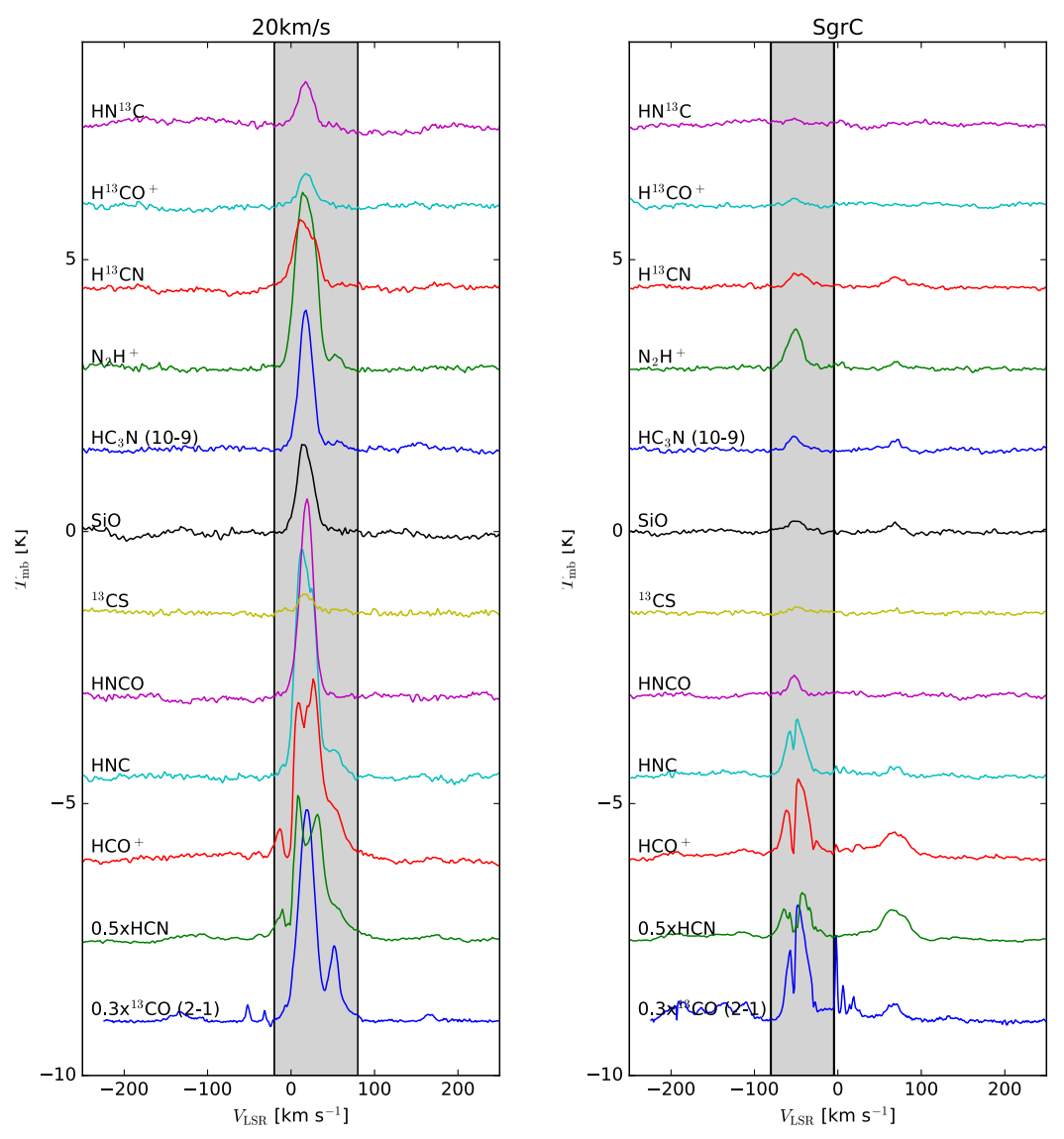

Fig. A.6. Average spectra for each region shown in Fig. A.4. ${ }^{13} \mathrm{CO}(2-1)$ spectra are scaled by a factor of 0.3 and $\mathrm{HCN}$ spectra are scaled by a factor of 0.5 .

\section{Appendix B: Beam efficiency for APEX observations}

Since the Galactic center molecular emission is extended in comparison with the beam size, we estimate the beam efficiency for the APEX telescope considering an extended source. Considering the main beam efficiencies measured in November 2012 using Jupiter ${ }^{8}$ (see Table B.1), we performed a least squares fit for the Ruze formula to get the values for $B_{0}$ and $\sigma\left(B_{0}=0.69\right.$, $\sigma=0.019)$.

$B_{\text {eff }}(\lambda)=B_{0} \times \exp \left(-(4 \times \pi \times \sigma / \lambda)^{2}\right)$.

Table B.1. Beam efficiencies for APEX telescope using Jupiter.

\begin{tabular}{|c|c|c|c|c|c|}
\hline $\begin{array}{c}\text { Frequency } \\
{[\mathrm{GHz}]} \\
\end{array}$ & Source & $\begin{array}{c}\text { Source } \\
\text { size } \\
{\left[{ }^{\prime \prime}\right]} \\
\end{array}$ & $\begin{array}{c}\text { Main beam } \\
\text { size } \\
{\left[{ }^{\prime \prime}\right]} \\
\end{array}$ & $\begin{array}{l}\text { Forward } \\
\text { efficiency }\end{array}$ & $\begin{array}{c}\text { Beam } \\
\text { efficiency }\end{array}$ \\
\hline 349 & Jupiter & 44.8 & 17.6 & 0.95 & 0.67 \\
\hline 466 & Jupiter & & 13.2 & 0.95 & 0.58 \\
\hline 691.9 & Jupiter & 47.4 & 8.9 & 0.95 & 0.49 \\
\hline 807.1 & Jupiter & & 7.7 & 0.95 & 0.48 \\
\hline
\end{tabular}

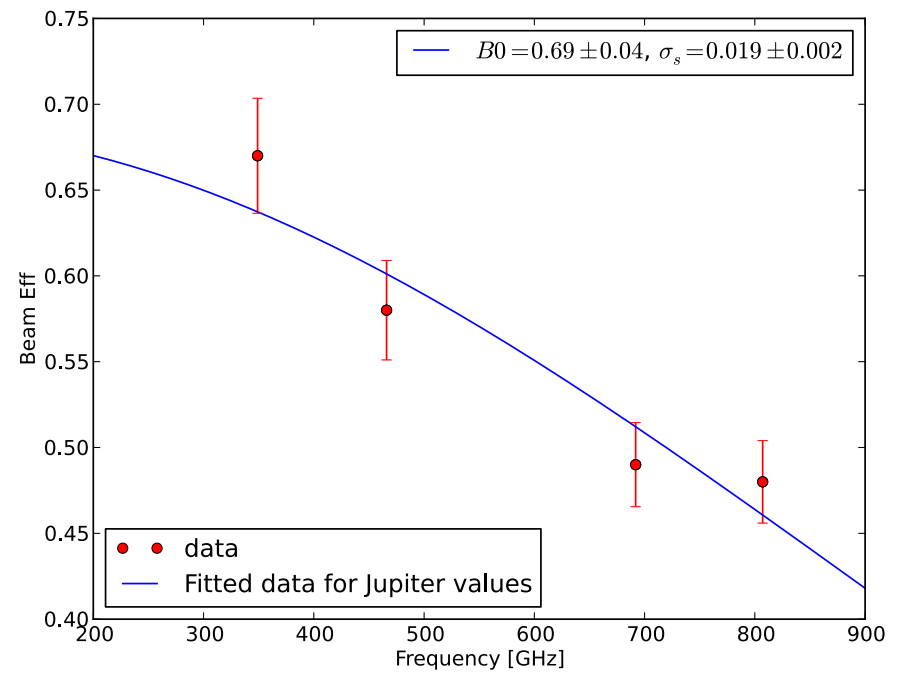

Fig. B.1. Least squares fit for the values of the main beam efficiency measured on November 2012 at the APEX telescope. We fit the Ruze formula to get the values for $B_{0}$ and $\sigma$. 
Appendix C: Velocity integrated emission

\section{$\mathbb{H}^{13} \mathrm{CN}$}

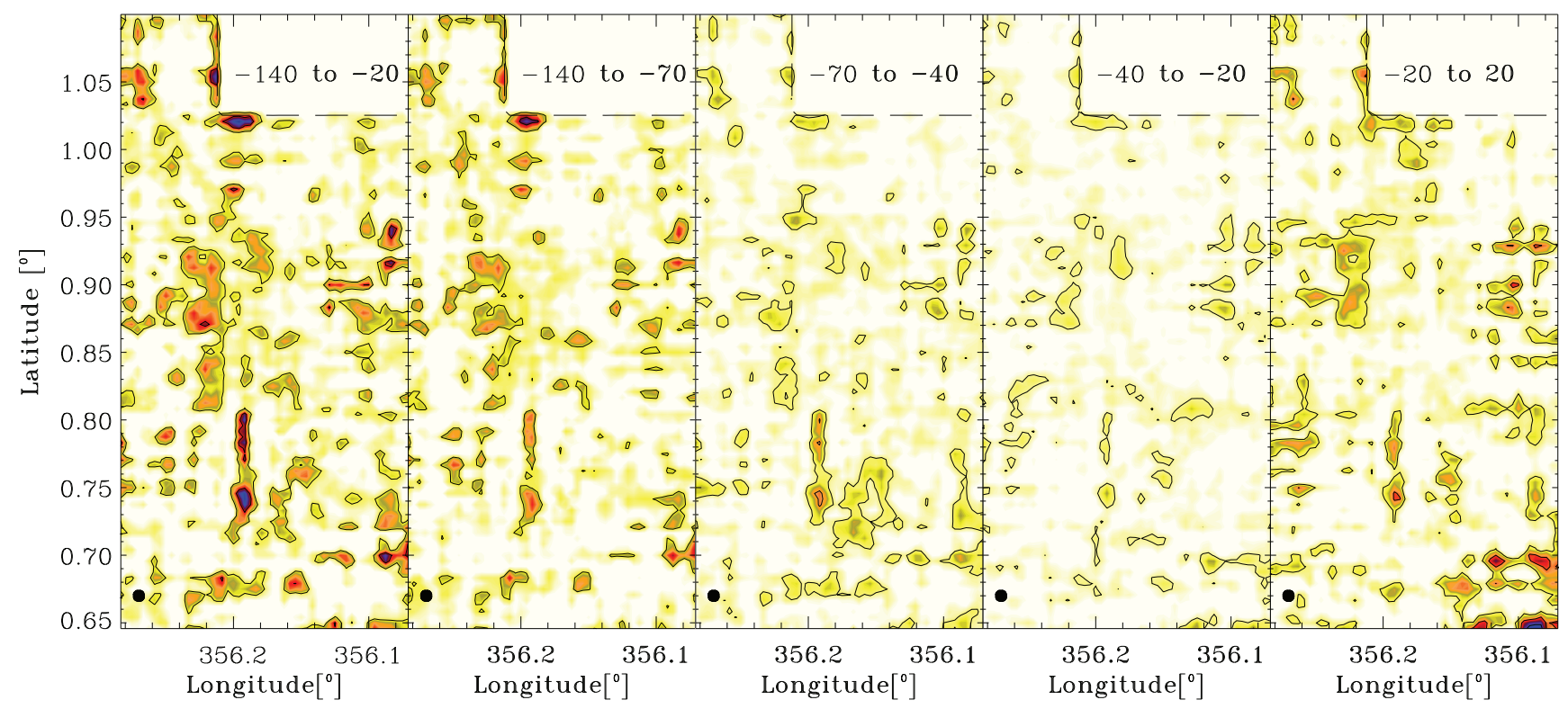

$\left[\begin{array}{lll}\mathrm{K} \mathrm{km} \mathrm{s} & -1\end{array}\right.$

0. 2. 4. 5. 7. 9.11

\begin{tabular}{lllllll}
\multicolumn{6}{c}{$\left[\mathrm{K} \mathrm{km} \mathrm{s}^{-1}\right]$} \\
\hline 0.00 & 1.23 & 2.47 & 3.70 & 4.93 & 6.17 & 7.40
\end{tabular}

Fig. C.1. Velocity integrated emission in $\mathrm{H}^{13} \mathrm{CN}$. From left to right: velocity range from -140 to $20 \mathrm{~km} \mathrm{~s}^{-1}$ (the complete velocity range covered by the GMLs); velocity range from -140 to $-70 \mathrm{~km} \mathrm{~s}^{-1} ;-70$ to $-40 \mathrm{~km} \mathrm{~s}^{-1},-40$ to $-20 \mathrm{~km} \mathrm{~s}^{-1}$ and -20 to $20 \mathrm{~km} \mathrm{~s}^{-1}$.

\section{$\mathrm{SiO}$}

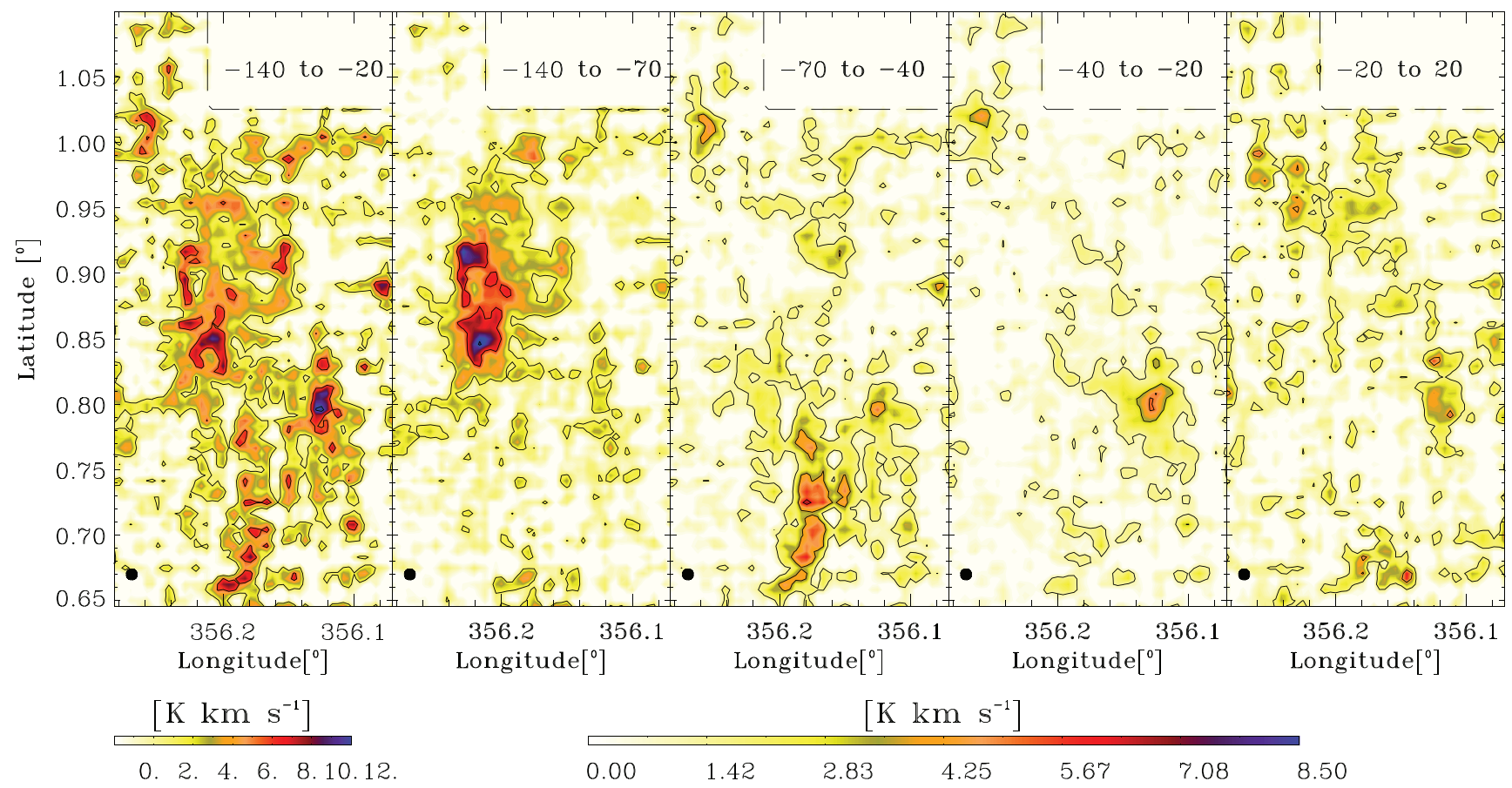

Fig. C.2. Velocity integrated emission in SiO. From left to right: velocity range from -140 to $20 \mathrm{~km} \mathrm{~s}^{-1}$ (the complete velocity range covered by the GMLs); velocity range from -140 to $-70 \mathrm{~km} \mathrm{~s}^{-1} ;-70$ to $-40 \mathrm{~km} \mathrm{~s}^{-1},-40$ to $-20 \mathrm{~km} \mathrm{~s}^{-1}$ and -20 to $20 \mathrm{~km} \mathrm{~s}^{-1}$. 


\section{HNCO}

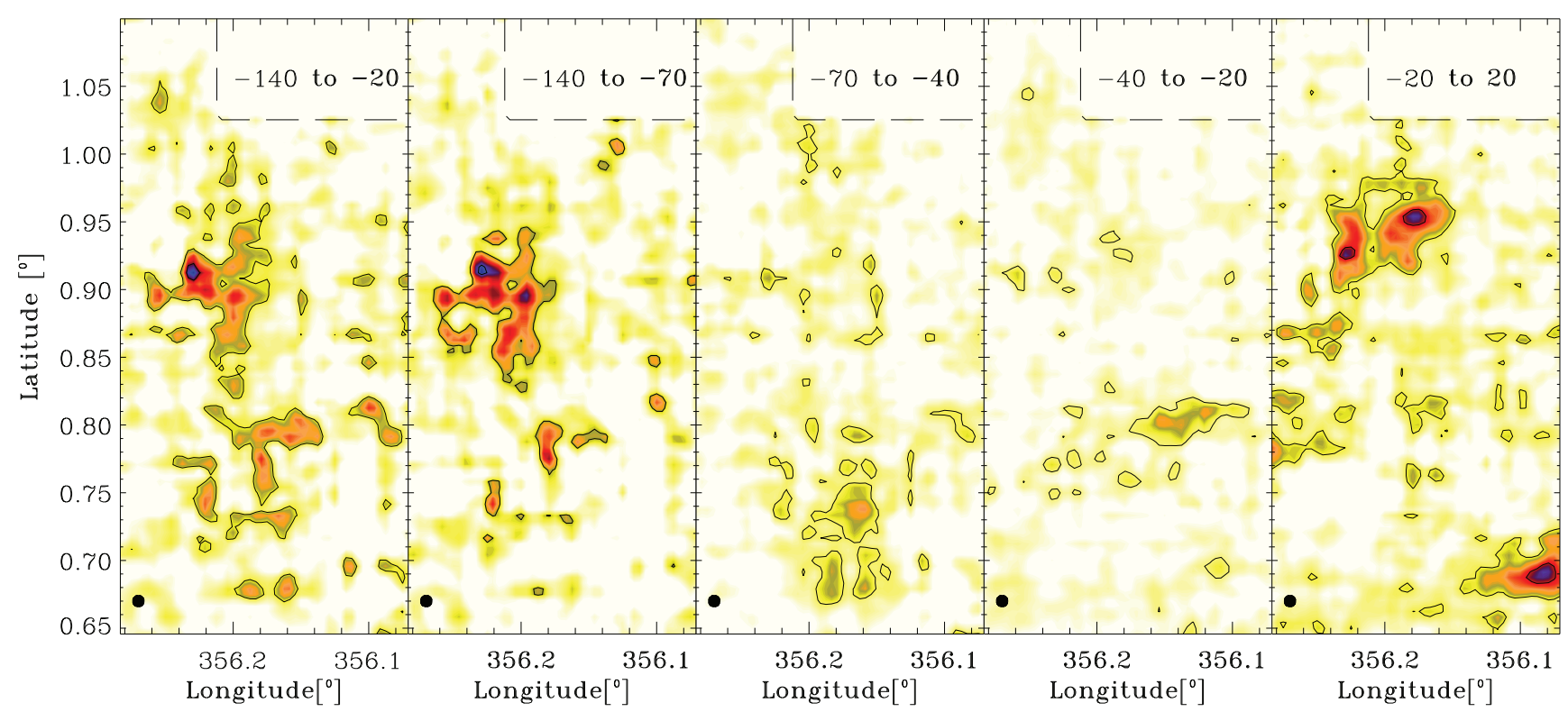

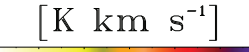

0. 2. 3. 5. 6. 8. 9

\begin{tabular}{lllllll}
\multicolumn{8}{c}{$\left[\mathrm{K} \mathrm{km} \mathrm{s}^{-1}\right]$} \\
\hline 0.00 & 0.98 & 1.97 & 2.95 & 3.93 & 4.92 & 5.90
\end{tabular}

Fig. C.3. Velocity integrated emission in HNCO. From left to right: velocity range from -140 to $20 \mathrm{~km} \mathrm{~s}^{-1}$ (the complete velocity range covered by the GMLs); velocity range from -140 to $-70 \mathrm{~km} \mathrm{~s}^{-1} ;-70$ to $-40 \mathrm{~km} \mathrm{~s}^{-1},-40$ to $-20 \mathrm{~km} \mathrm{~s}^{-1}$ and -20 to $20 \mathrm{~km} \mathrm{~s}^{-1}$.

\section{$\mathbb{H C N}$}

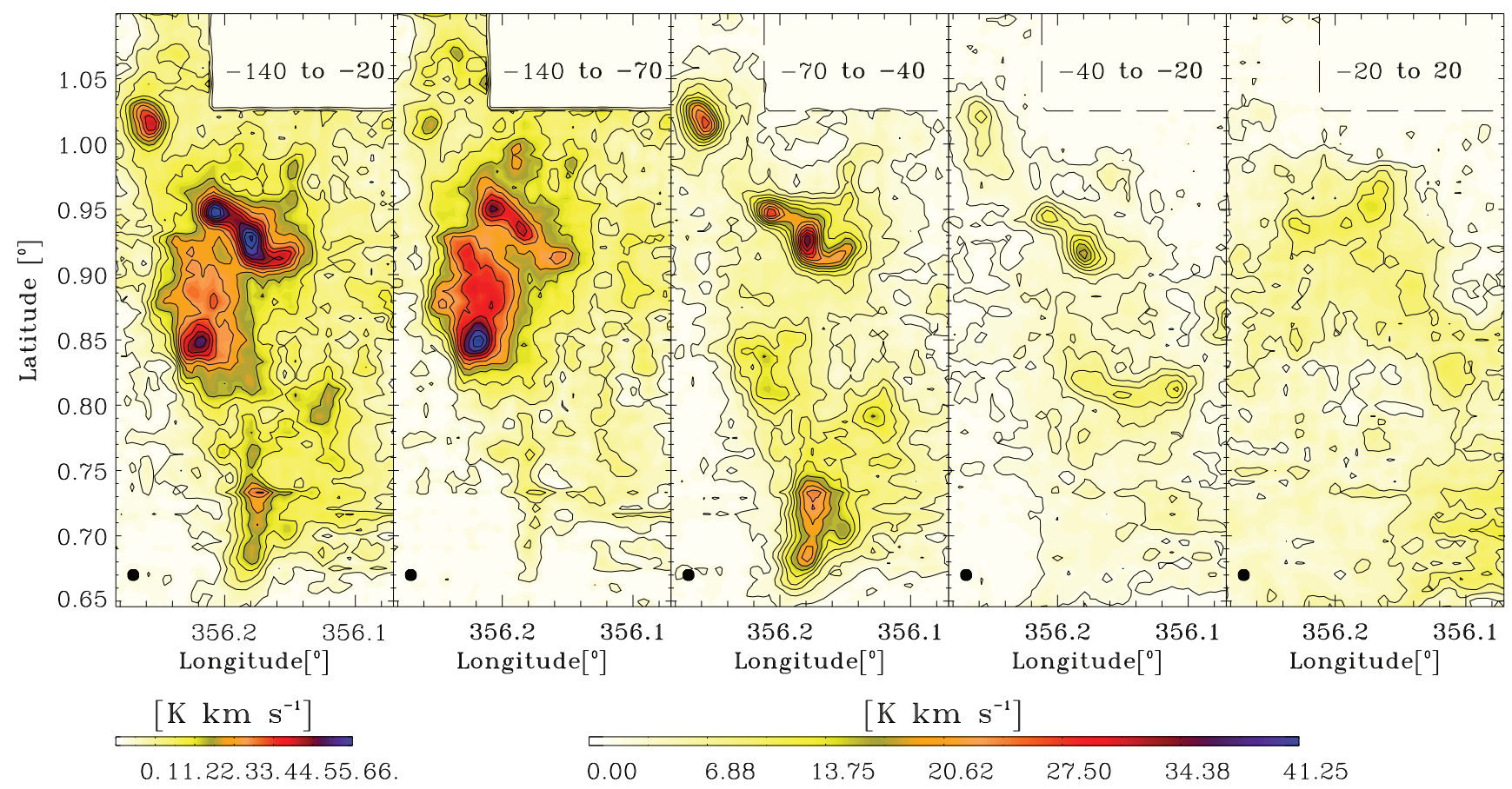

Fig. C.4. Velocity integrated emission in HCN. From left to right: velocity range from -140 to $20 \mathrm{~km} \mathrm{~s}^{-1}$ (the complete velocity range covered by the GMLs); velocity range from -140 to $-70 \mathrm{~km} \mathrm{~s}^{-1} ;-70$ to $-40 \mathrm{~km} \mathrm{~s}^{-1},-40$ to $-20 \mathrm{~km} \mathrm{~s}^{-1}$ and -20 to $20 \mathrm{~km} \mathrm{~s}^{-1}$. 


\section{$\mathrm{HCO}^{+}$}

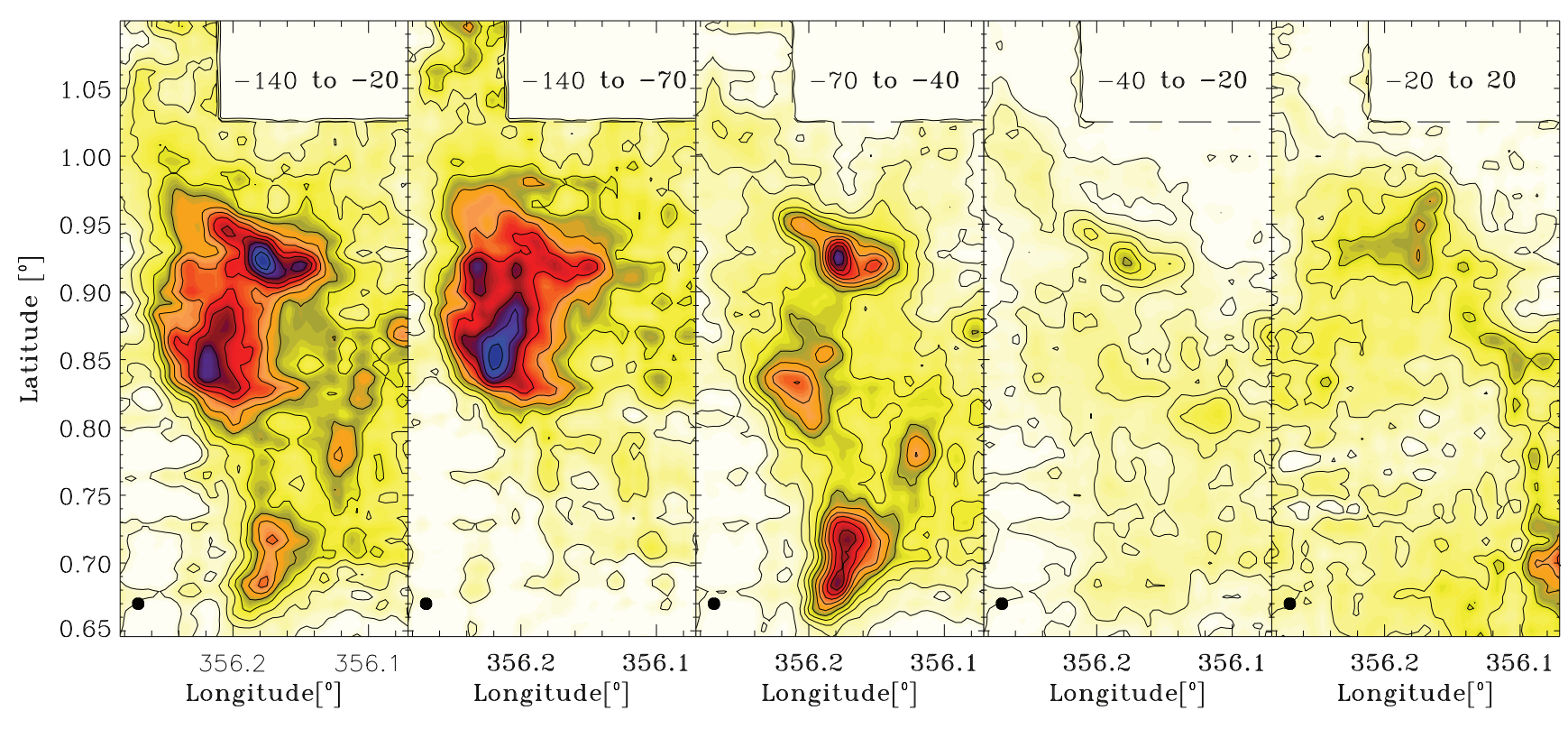

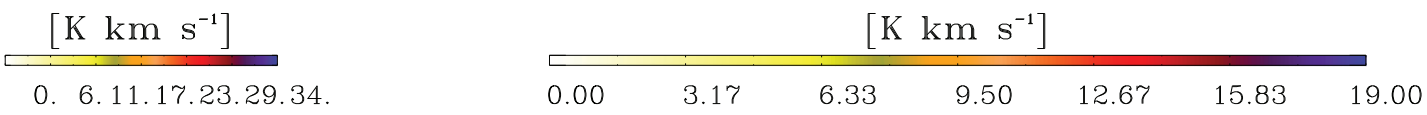

Fig. C.5. Velocity integrated emission in $\mathrm{HCO}^{+}$. From left to right: velocity range from -140 to $20 \mathrm{~km} \mathrm{~s}^{-1}$ (the complete velocity range covered by the GMLs); velocity range from -110 to $-70 \mathrm{~km} \mathrm{~s}^{-1} ;-70$ to $-40 \mathrm{~km} \mathrm{~s}^{-1},-40$ to $-20 \mathrm{~km} \mathrm{~s}^{-1}$ and -20 to $20 \mathrm{~km} \mathrm{~s}^{-1}$.

HNC

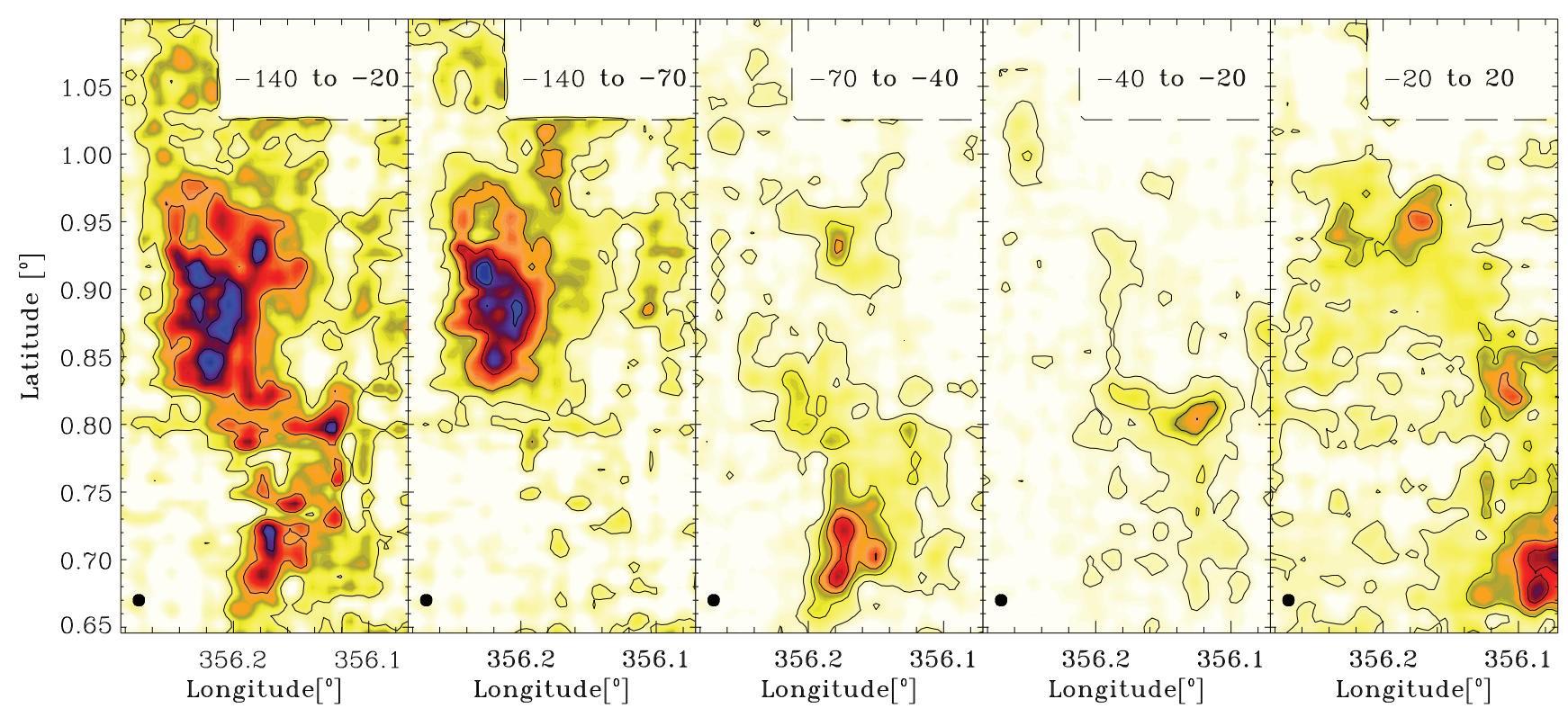

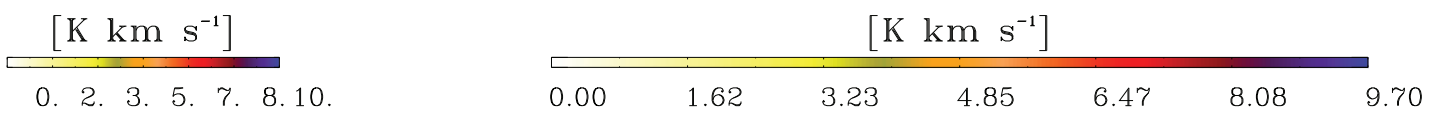

Fig. C.6. Velocity integrated emission in HNC. From left to right: velocity range from -140 to $20 \mathrm{~km} \mathrm{~s}^{-1}$ (the complete velocity range covered by the GMLs); velocity range from -140 to $-70 \mathrm{~km} \mathrm{~s}^{-1} ;-70$ to $-40 \mathrm{~km} \mathrm{~s}^{-1},-40$ to $-20 \mathrm{~km} \mathrm{~s}^{-1}$ and -20 to $20 \mathrm{~km} \mathrm{~s}^{-1}$. 
$\mathrm{HC}_{3} \mathrm{~N}(10-9)$

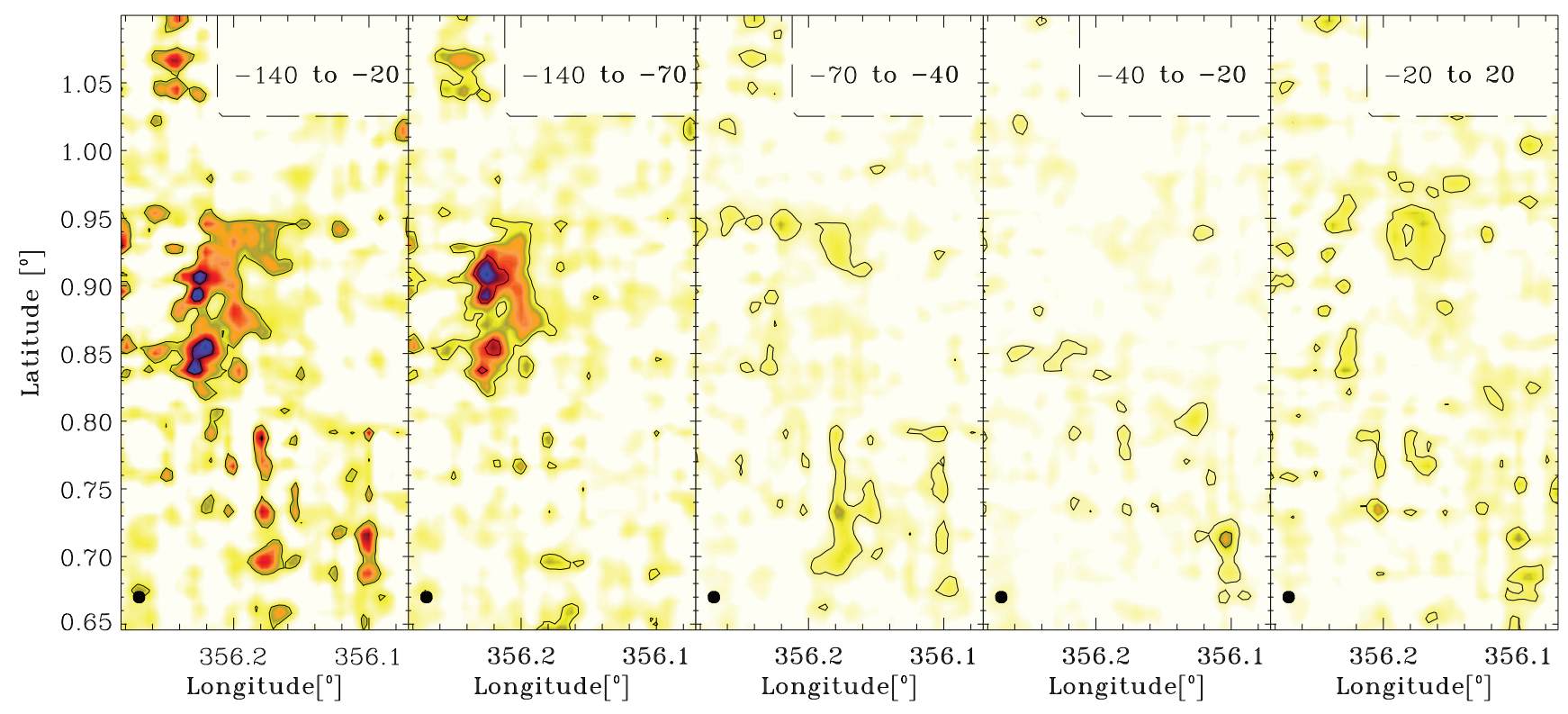

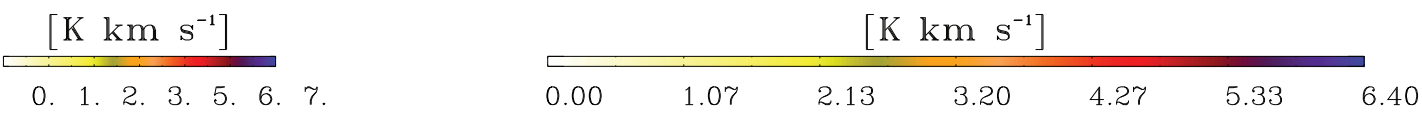

Fig. C.7. Velocity integrated emission in $\mathrm{HC}_{3} \mathrm{~N}(10-9)$. From left to right: velocity range from -140 to $20 \mathrm{~km} \mathrm{~s}^{-1}$ (the complete velocity range covered by the GMLs); velocity range from -140 to $-70 \mathrm{~km} \mathrm{~s}^{-1} ;-70$ to $-40 \mathrm{~km} \mathrm{~s}^{-1},-40$ to $-20 \mathrm{~km} \mathrm{~s}^{-1}$ and -20 to $20 \mathrm{~km} \mathrm{~s}$.

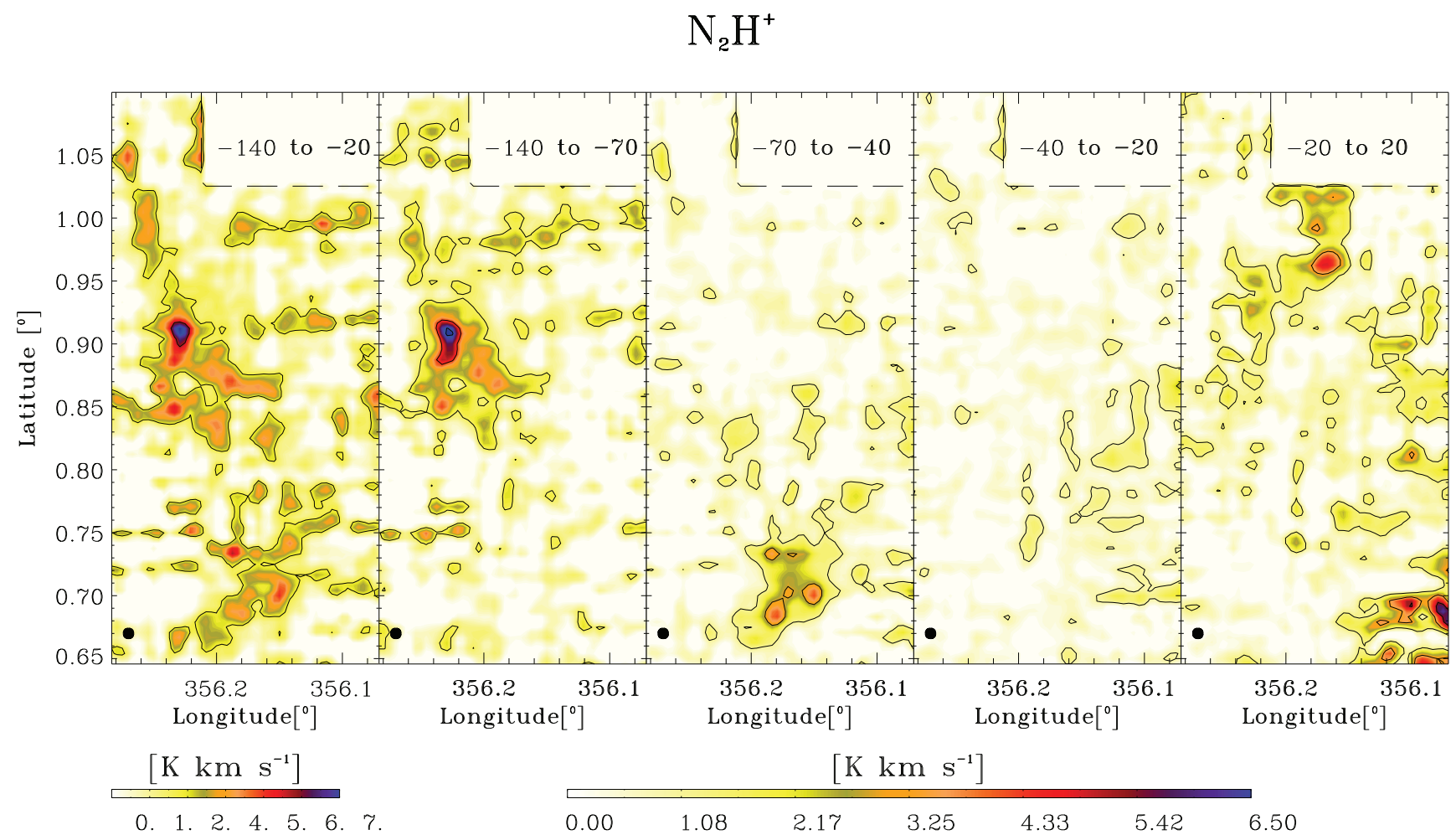

Fig. C.8. Velocity integrated emission in $\mathrm{N}_{2} \mathrm{H}^{+}$. From left to right: velocity range from -140 to $20 \mathrm{~km} \mathrm{~s}^{-1}$ (the complete velocity range covered by the GMLs); velocity range from -140 to $-70 \mathrm{~km} \mathrm{~s}^{-1} ;-70$ to $-40 \mathrm{~km} \mathrm{~s}^{-1},-40$ to $-20 \mathrm{~km} \mathrm{~s}^{-1}$ and -20 to $20 \mathrm{~km} \mathrm{~s}^{-1}$. 


\section{$\mathrm{CH}_{3} \mathrm{OH}(2-1)$}

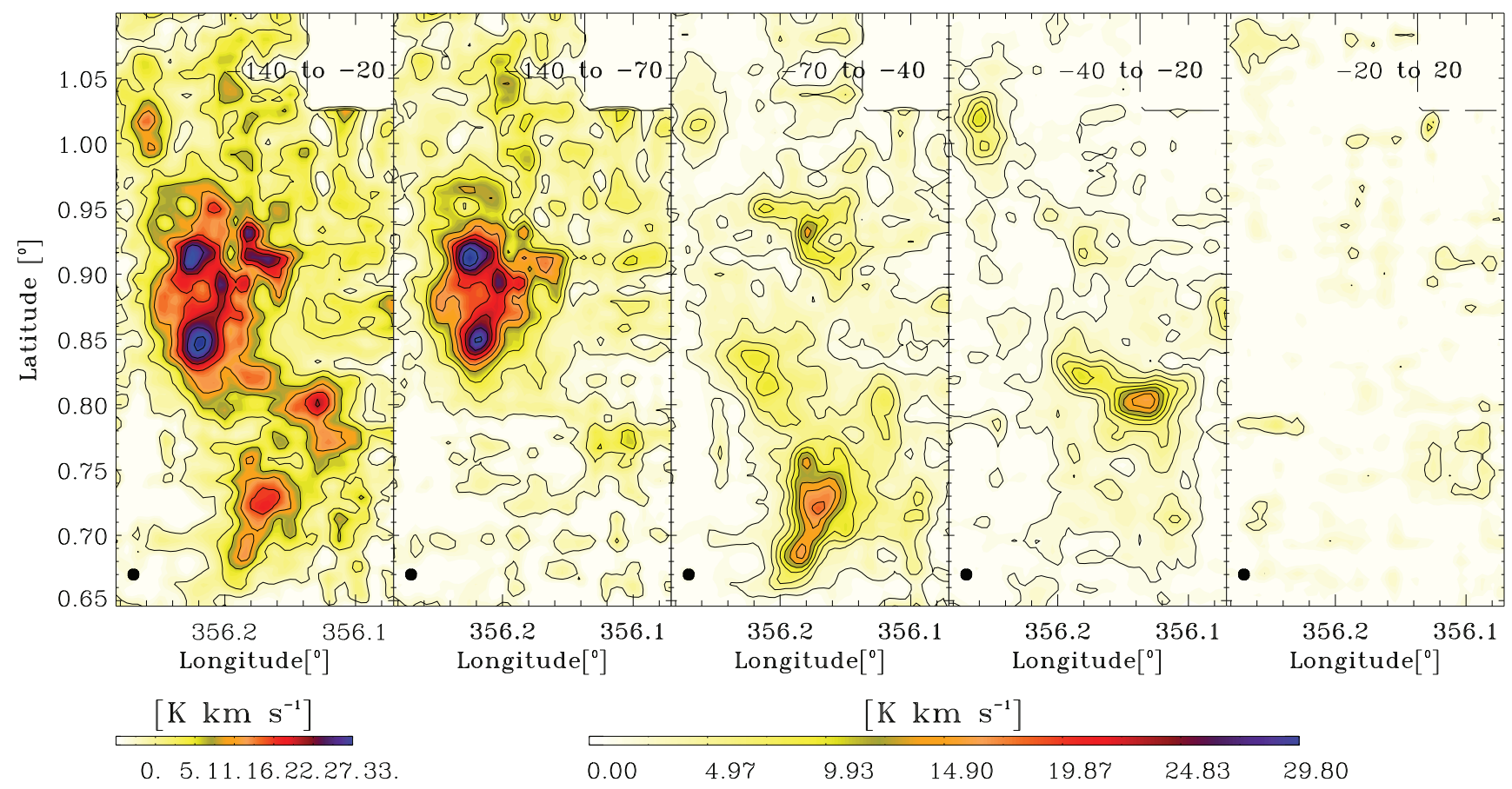

Fig. C.9. Velocity integrated emission in $\mathrm{CH}_{3} \mathrm{OH}$. From left to right: velocity range from -140 to $20 \mathrm{~km} \mathrm{~s}^{-1}$ (the complete velocity range covered by the GMLs); velocity range from -140 to $-70 \mathrm{~km} \mathrm{~s}^{-1} ;-70$ to $-40 \mathrm{~km} \mathrm{~s}^{-1},-40$ to $-20 \mathrm{~km} \mathrm{~s}^{-1}$ and -20 to $20 \mathrm{~km} \mathrm{~s}^{-1}$.

\section{OCS}

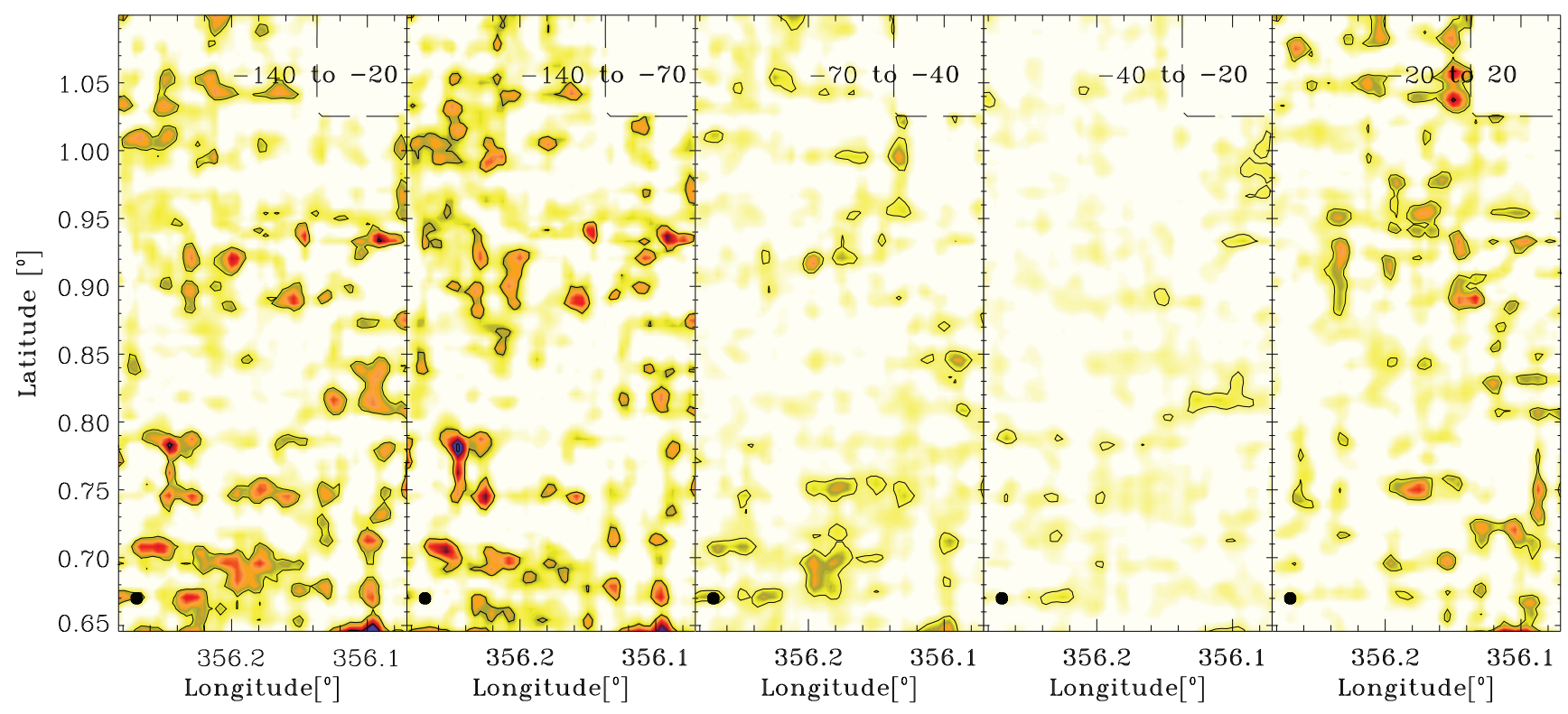

$\left[\mathrm{K} \mathrm{km} \mathrm{s}^{-1}\right]$

0. 2. 3. 5. 6. 8. 10

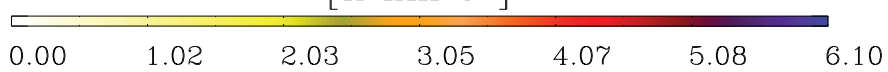

Fig. C.10. Velocity integrated emission in OCS. From left to right: velocity range from -140 to $20 \mathrm{~km} \mathrm{~s}^{-1}$ (the complete velocity range covered by the GMLs); velocity range from -140 to $-70 \mathrm{~km} \mathrm{~s}^{-1} ;-70$ to $-40 \mathrm{~km} \mathrm{~s}^{-1},-40$ to $-20 \mathrm{~km} \mathrm{~s}^{-1}$ and -20 to $20 \mathrm{~km} \mathrm{~s}^{-1}$. 


\section{CS}

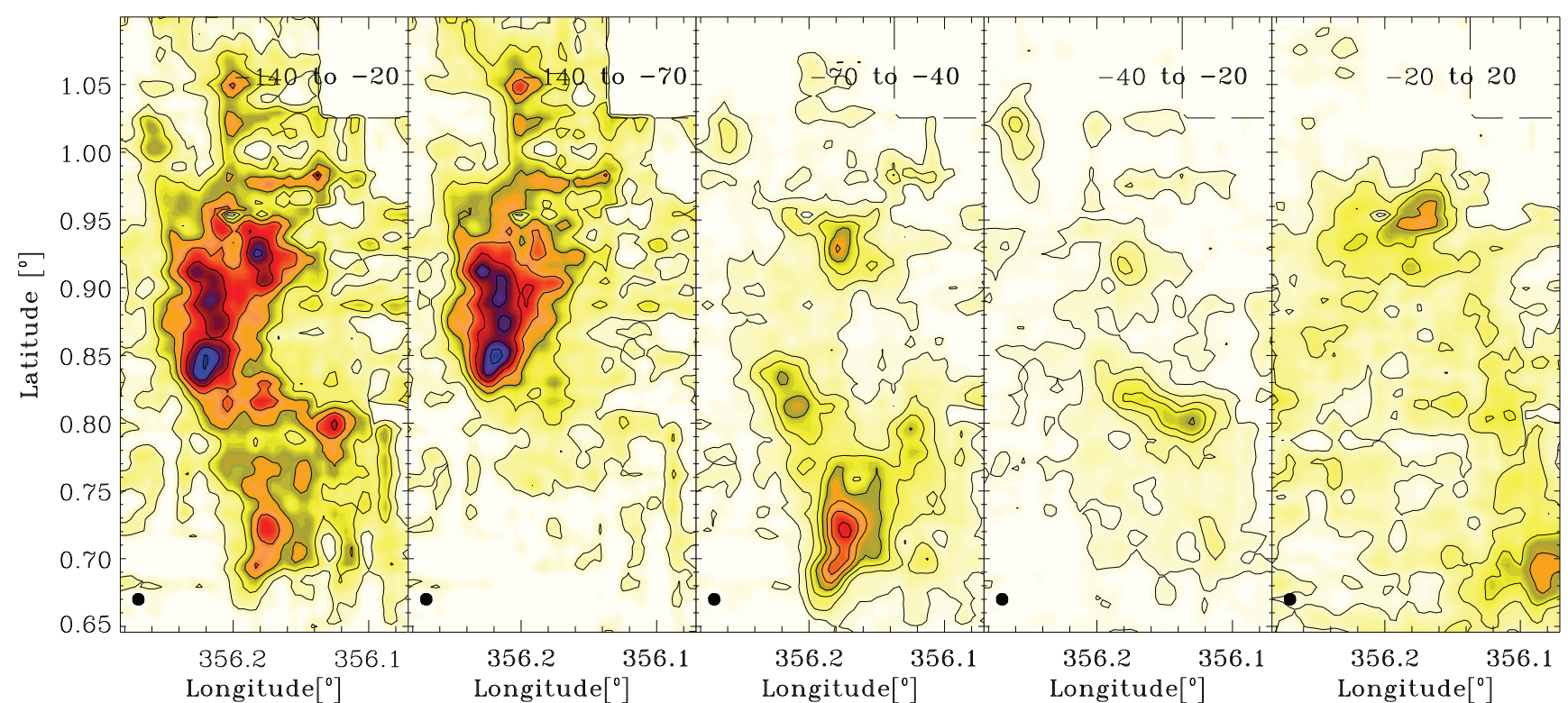

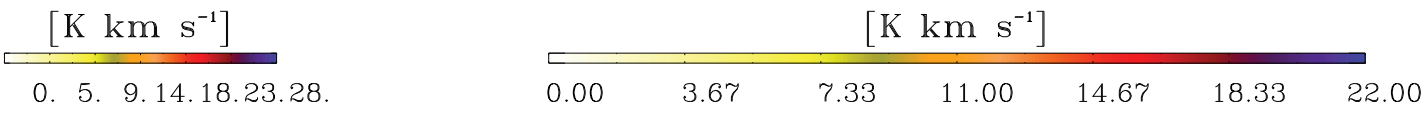

Fig. C.11. Velocity integrated emission in CS. From left to right: velocity range from -140 to $20 \mathrm{~km} \mathrm{~s}^{-1}$ (the complete velocity range covered by the GMLs); velocity range from -140 to $-70 \mathrm{~km} \mathrm{~s}^{-1} ;-70$ to $-40 \mathrm{~km} \mathrm{~s}^{-1},-40$ to $-20 \mathrm{~km} \mathrm{~s}^{-1}$ and -20 to $20 \mathrm{~km} \mathrm{~s}^{-1}$.

SO $3(2)-2(1)$

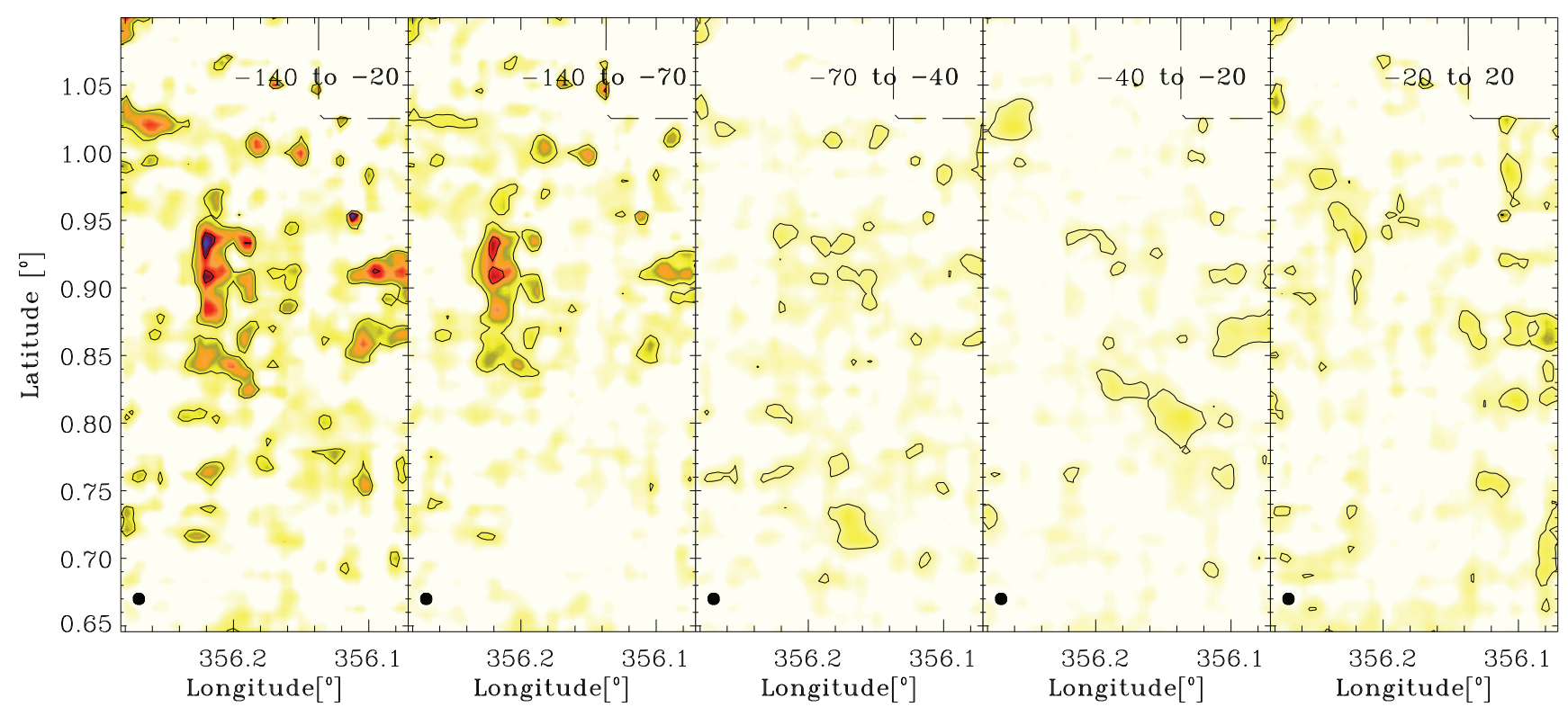

$\left[\mathrm{K} \mathrm{km} \mathrm{s}^{-1}\right]$

0. 2. 4. 6. 8. 10.11

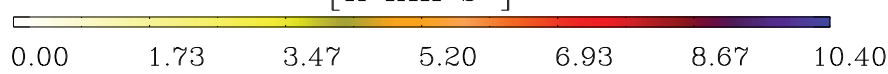

Fig. C.12. Velocity integrated emission in $\mathrm{SO}\left(3_{2}-2_{1}\right)$. From left to right: velocity range from -140 to $20 \mathrm{~km} \mathrm{~s}^{-1}$ (the complete velocity range covered by the GMLs); velocity range from -140 to $-70 \mathrm{~km} \mathrm{~s}^{-1} ;-70$ to $-40 \mathrm{~km} \mathrm{~s}^{-1},-40$ to $-20 \mathrm{~km} \mathrm{~s}^{-1}$ and -20 to $20 \mathrm{~km} \mathrm{~s}^{-1}$. 
$\mathrm{HC}_{3} \mathrm{~N}(11-10)$

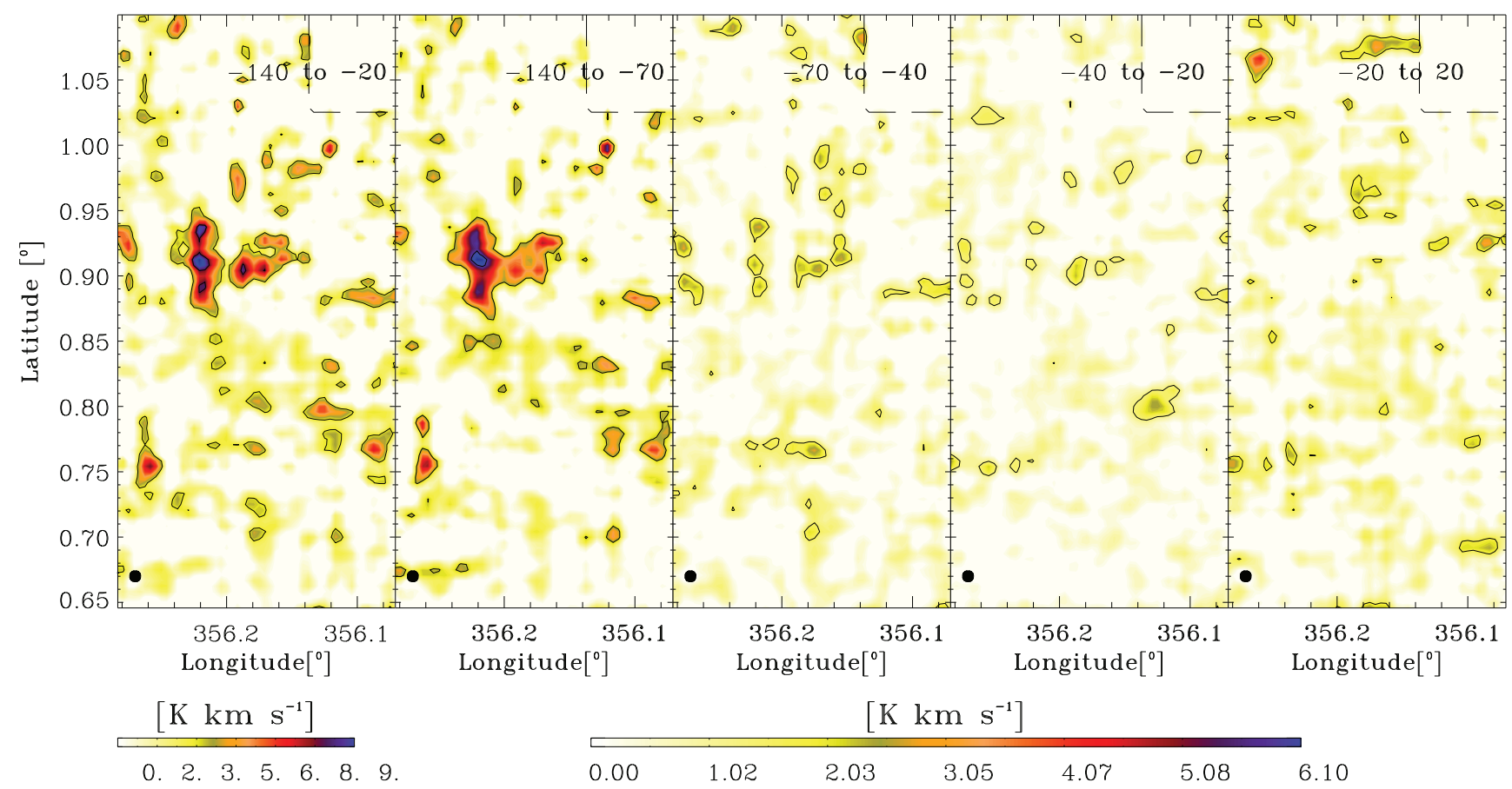

Fig. C.13. Velocity integrated emission in $\mathrm{HC}_{3} \mathrm{~N}(11-10)$. From left to right: velocity range from -140 to $20 \mathrm{~km} \mathrm{~s}^{-1}$ (the complete velocity range covered by the GMLs); velocity range from -140 to $-70 \mathrm{~km} \mathrm{~s}^{-1} ;-70$ to $-40 \mathrm{~km} \mathrm{~s}^{-1},-40$ to $-20 \mathrm{~km} \mathrm{~s}^{-1}$ and -20 to $20 \mathrm{~km} \mathrm{~s}^{-1}$.

\section{Appendix D: Latitude-velocity maps}

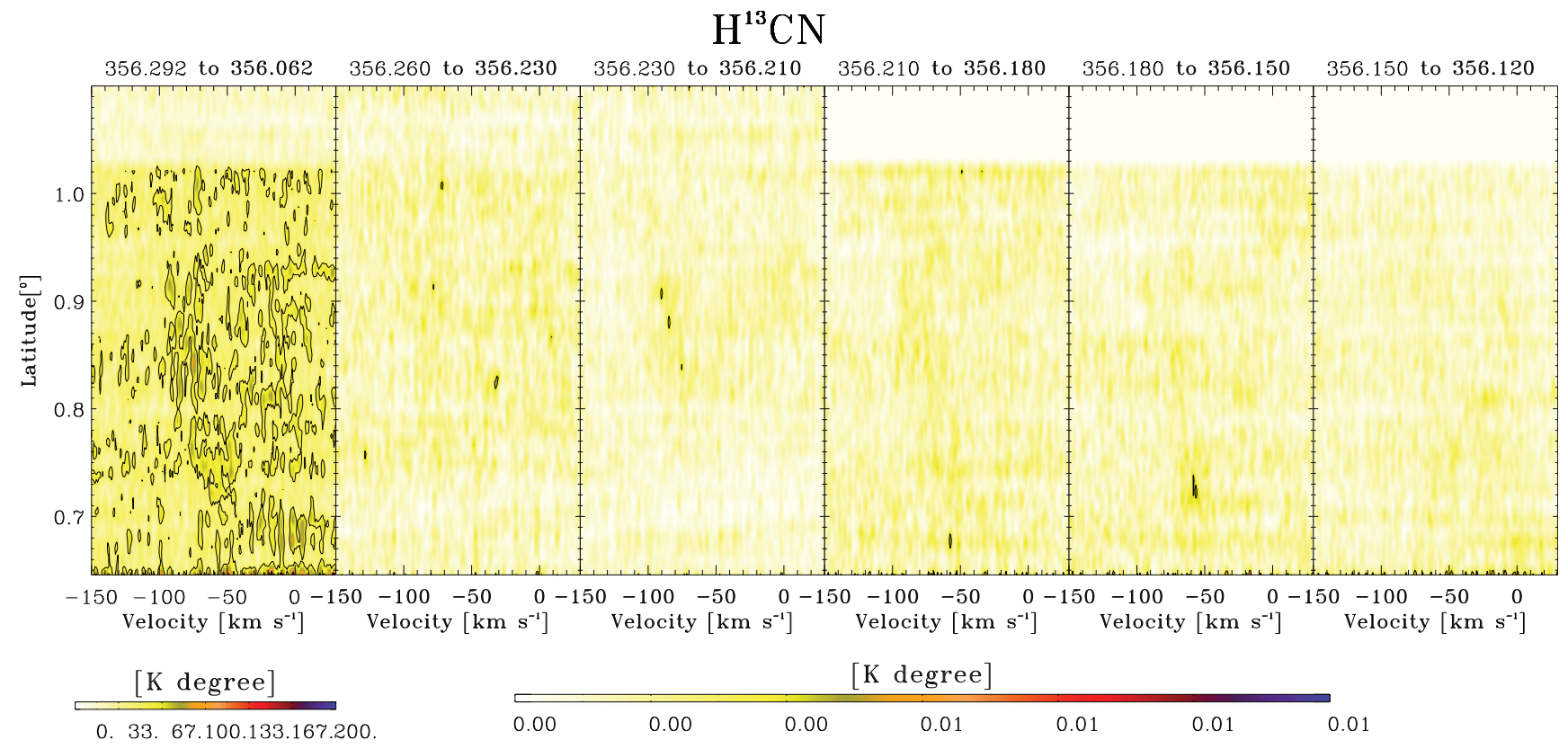

Fig. D.1. Latitude-velocity maps of the $\mathrm{H}^{13} \mathrm{CN}$ emission integrated in the complete longitude range from $356^{\circ} .29165$ to $356^{\circ} .06249$ (left panel), and in longitude steps of 108" (subsequent panels). 
$\mathrm{SiO}$

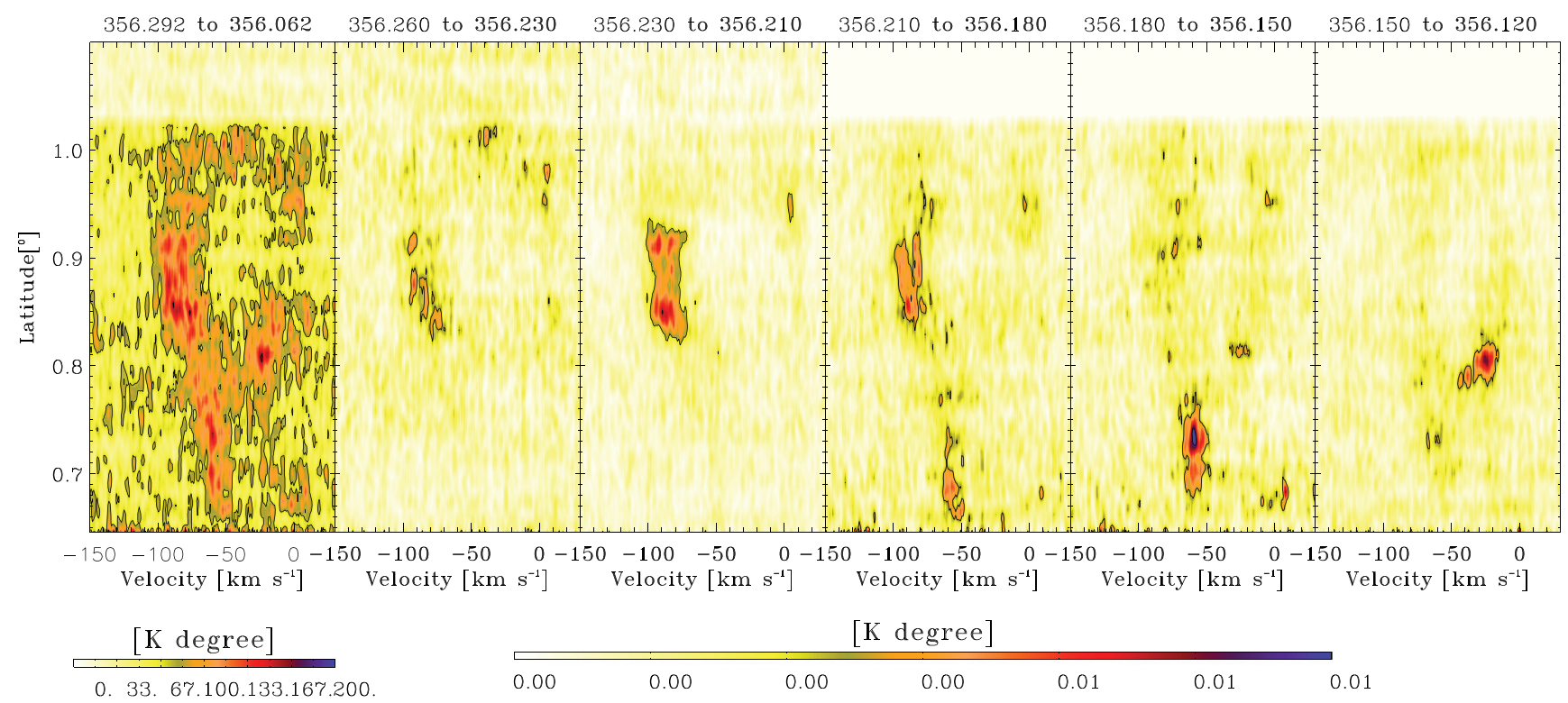

Fig. D.2. Latitude-velocity maps of the $\mathrm{SiO}$ emission integrated in the complete longitude range from $356^{\circ} .29165$ to $356^{\circ} .06249$ (left panel), and in longitude steps of $108^{\prime \prime}$ (subsequent panels).

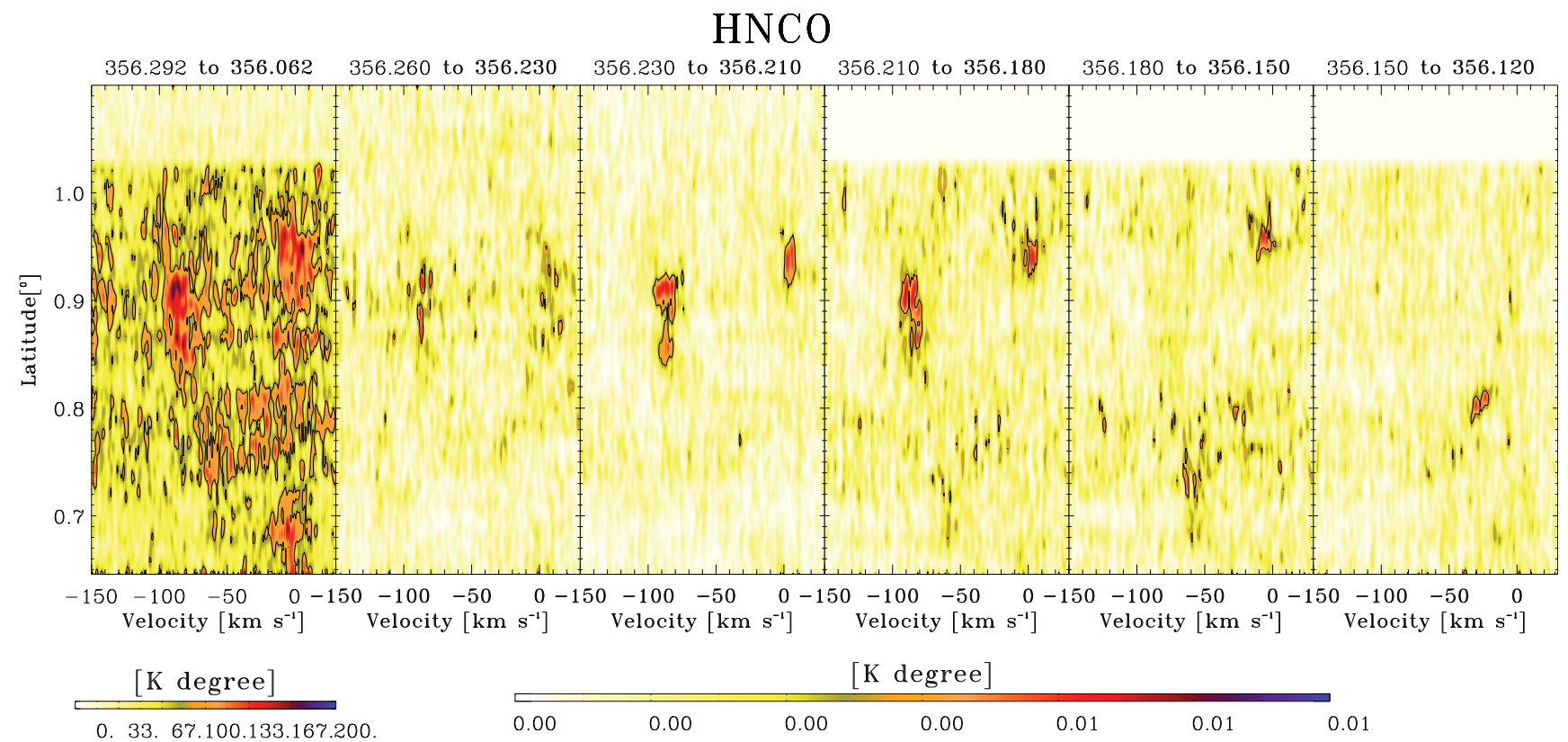

Fig. D.3. Latitude-velocity maps of the HNCO emission integrated in the complete longitude range from $356^{\circ} .29165$ to $356^{\circ} .06249$ (left panel), and in longitude steps of $108^{\prime \prime}$ (subsequent panels). 


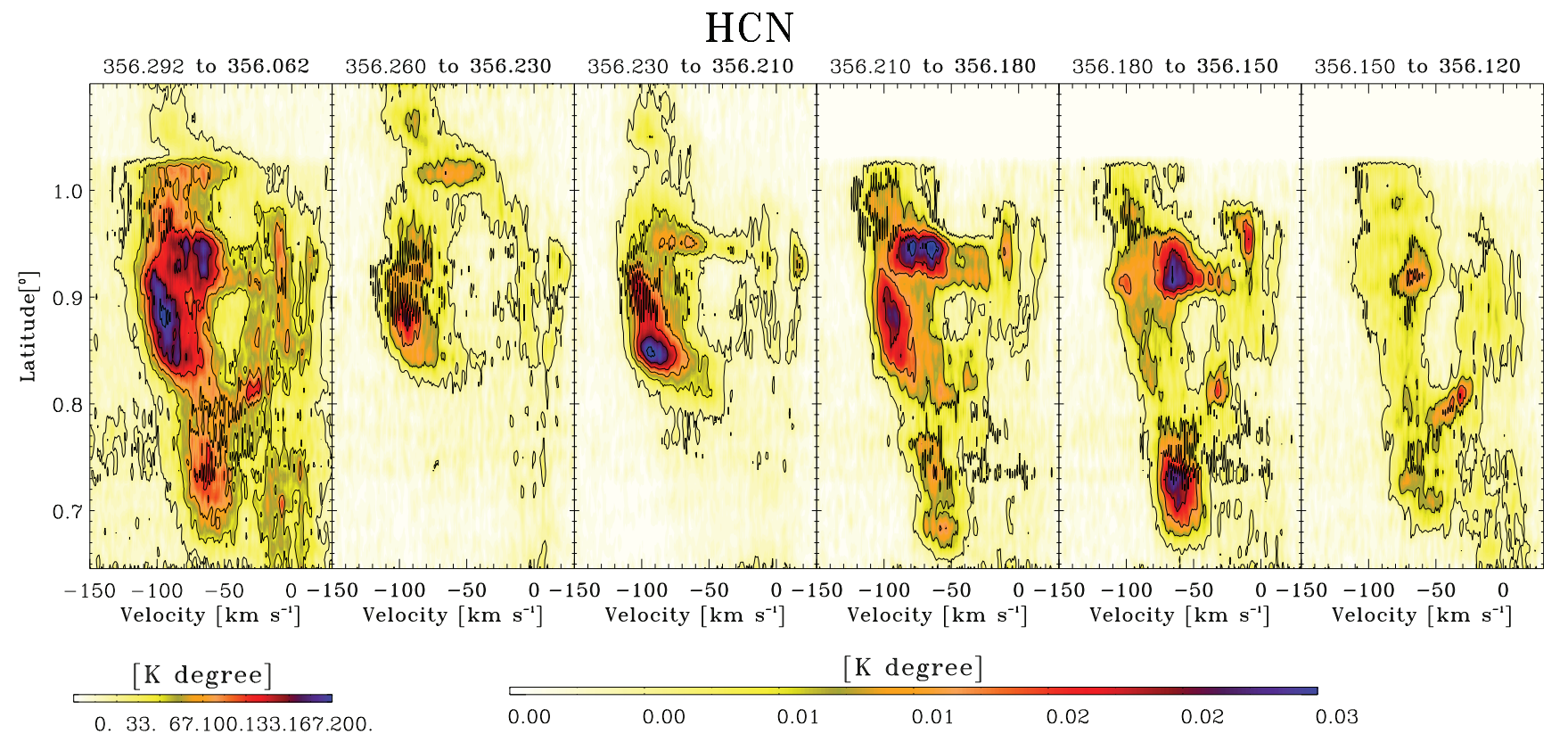

Fig. D.4. Latitude-velocity maps of the $\mathrm{HCN}$ emission integrated in the complete longitude range from $356^{\circ} .29165$ to $356^{\circ} .06249$ (left panel), and in longitude steps of 108" (subsequent panels).

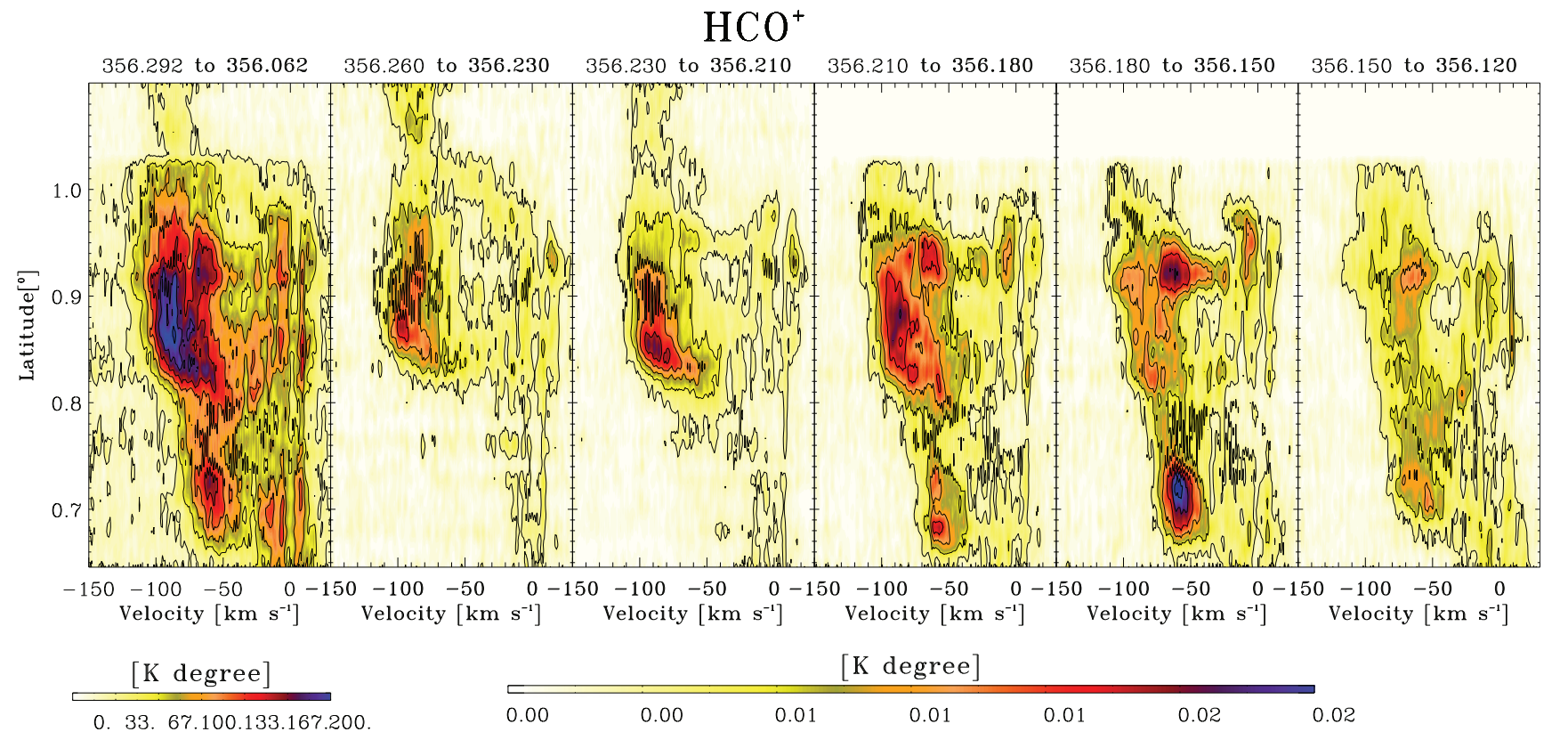

Fig. D.5. Latitude-velocity maps of the $\mathrm{HCO}^{+}$emission integrated in the complete longitude range from $356^{\circ} .29165$ to $356^{\circ} .06249$ (left panel), and in longitude steps of $108^{\prime \prime}$ (subsequent panels). 
$\mathbb{H N C}$

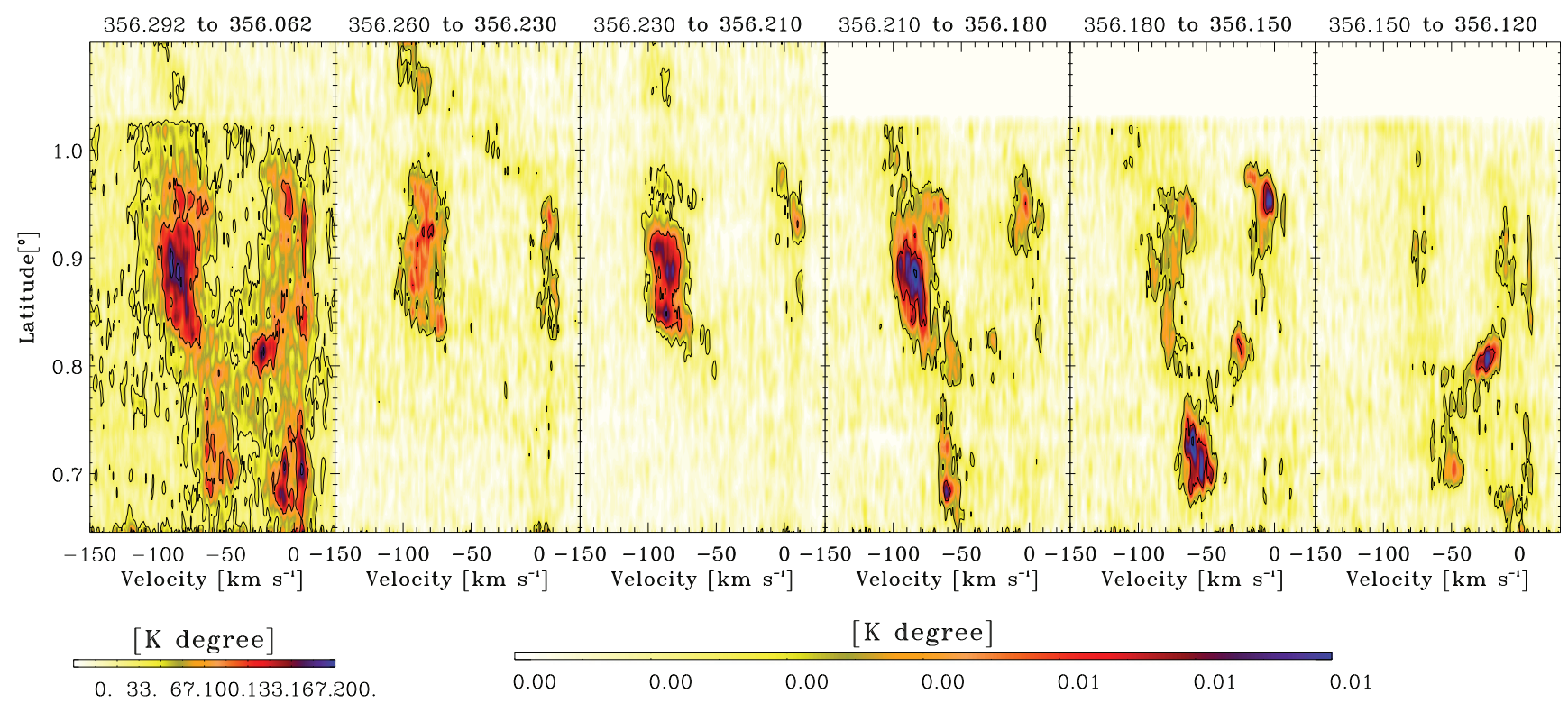

Fig. D.6. Latitude-velocity maps of the HNC emission integrated in the complete longitude range from $356^{\circ} .29165$ to $356^{\circ} .06249$ (left panel), and in longitude steps of $108^{\prime \prime}$ (subsequent panels).

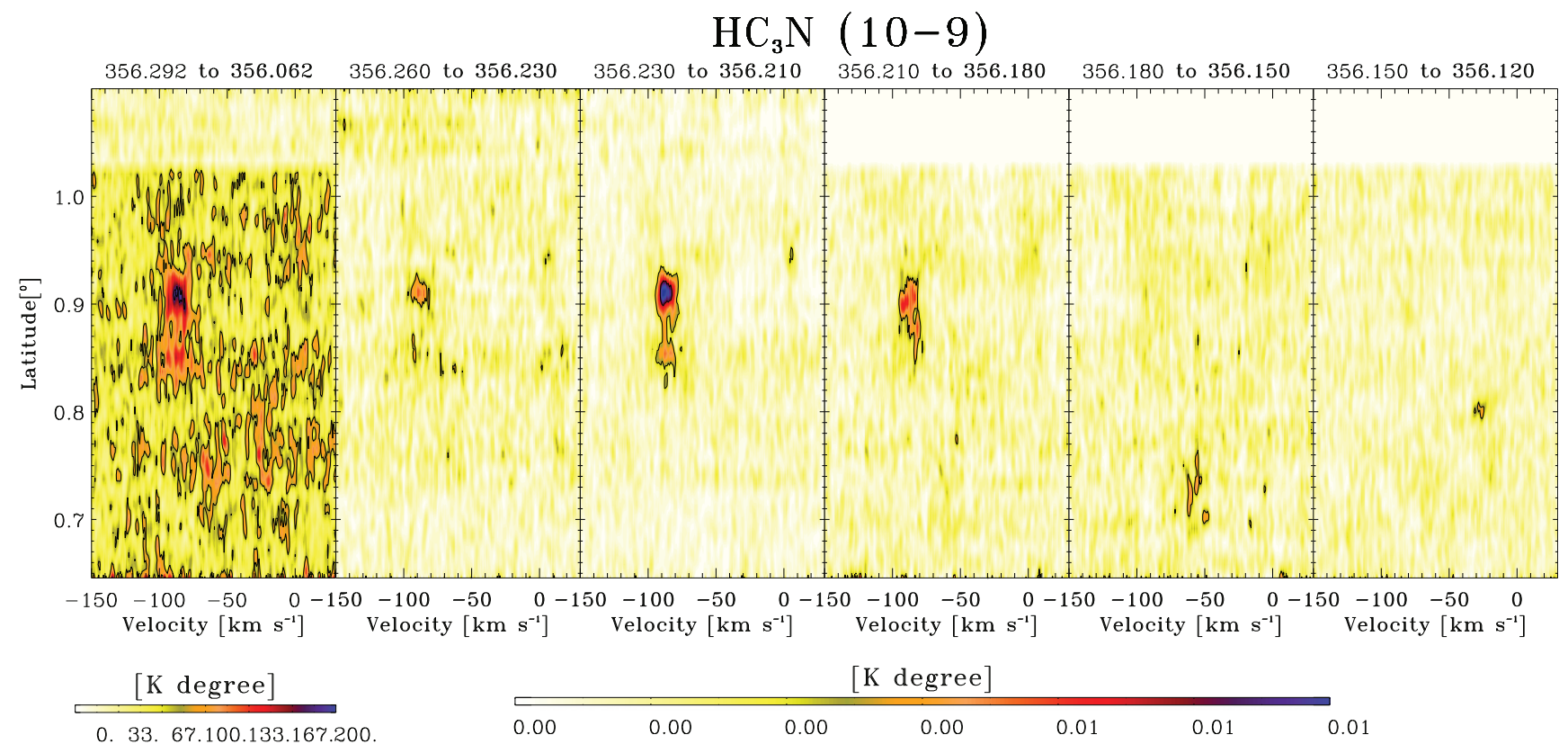

Fig. D.7. Latitude-velocity maps of the $\mathrm{HC}_{3} \mathrm{~N}(10-9)$ emission integrated in the complete longitude range from $356^{\circ} .29165$ to $356^{\circ} .06249$ (left panel), and in longitude steps of $108^{\prime \prime}$ (subsequent panels). 


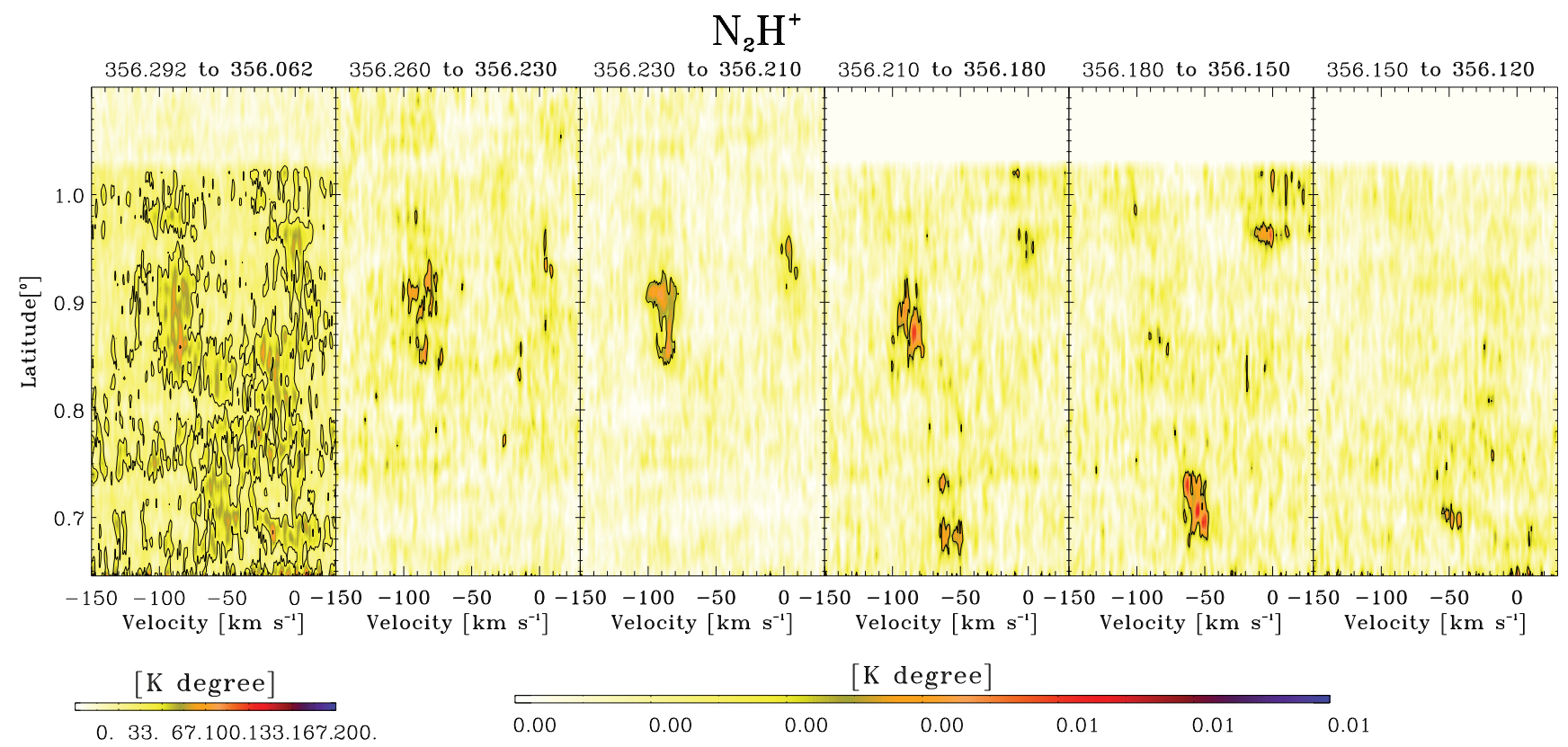

Fig. D.8. Latitude-velocity maps of the $\mathrm{N}_{2} \mathrm{H}^{+}$emission integrated in the complete longitude range from $356^{\circ} .29165$ to $356^{\circ} .06249$ (left panel), and in longitude steps of 108" (subsequent panels).

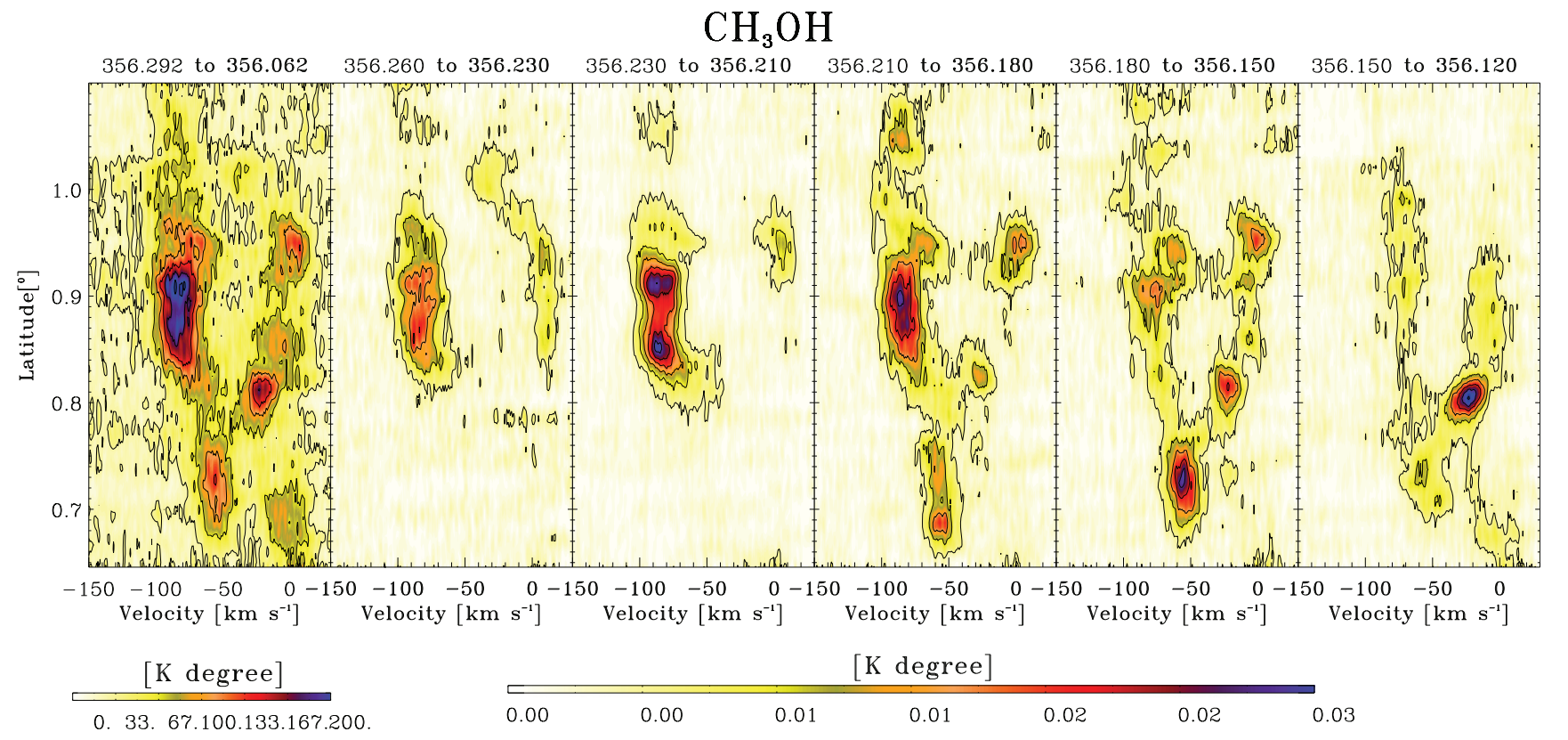

Fig. D.9. Latitude-velocity maps of the $\mathrm{CH}_{3} \mathrm{OH}$ emission integrated in the complete longitude range from $356^{\circ} .29165$ to $356^{\circ} .06249$ (left panel), and in longitude steps of $108^{\prime \prime}$ (subsequent panels). 


\section{OCS}

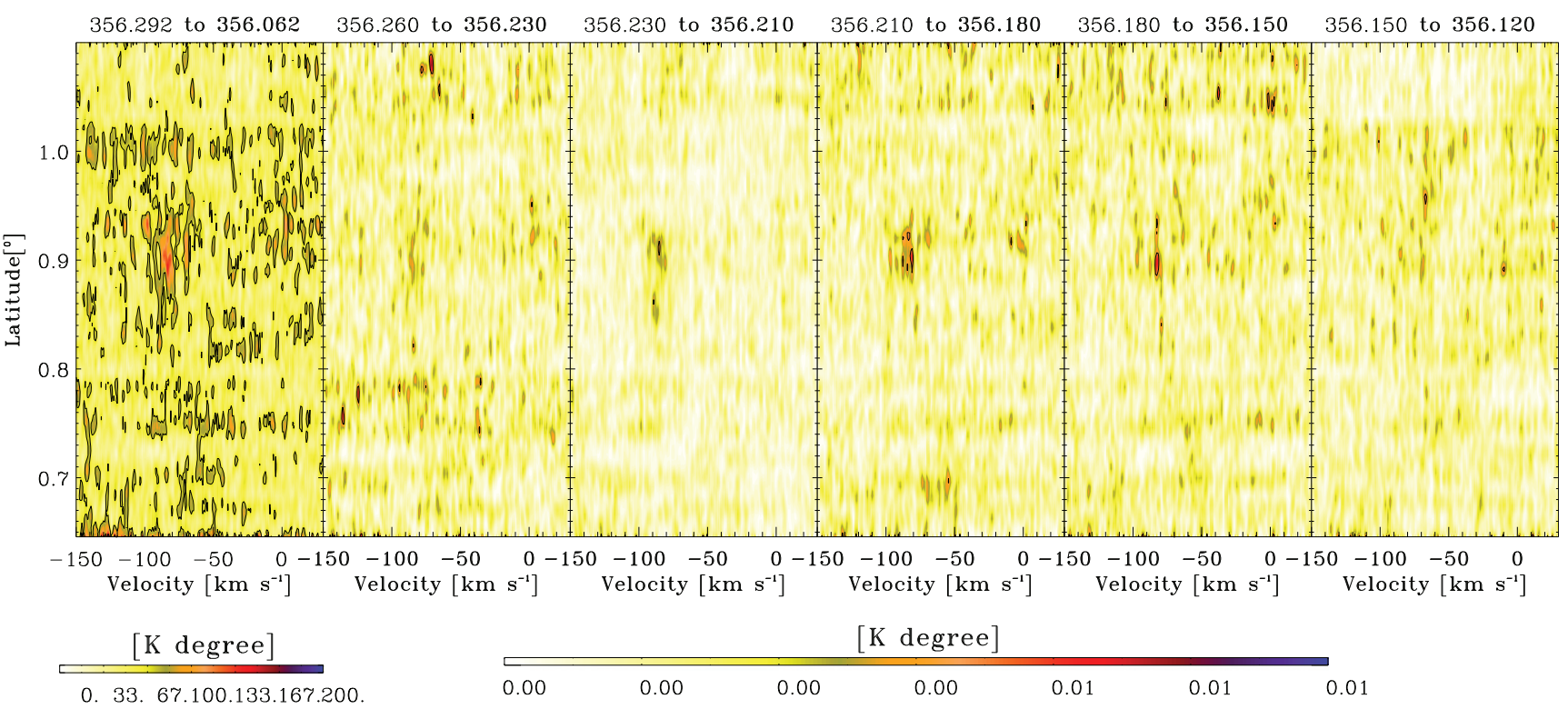

Fig. D.10. Latitude-velocity maps of the OCS emission integrated in the complete longitude range from $356^{\circ} .29165$ to $356^{\circ} .06249$ (left panel), and in longitude steps of 108" (subsequent panels).

CS

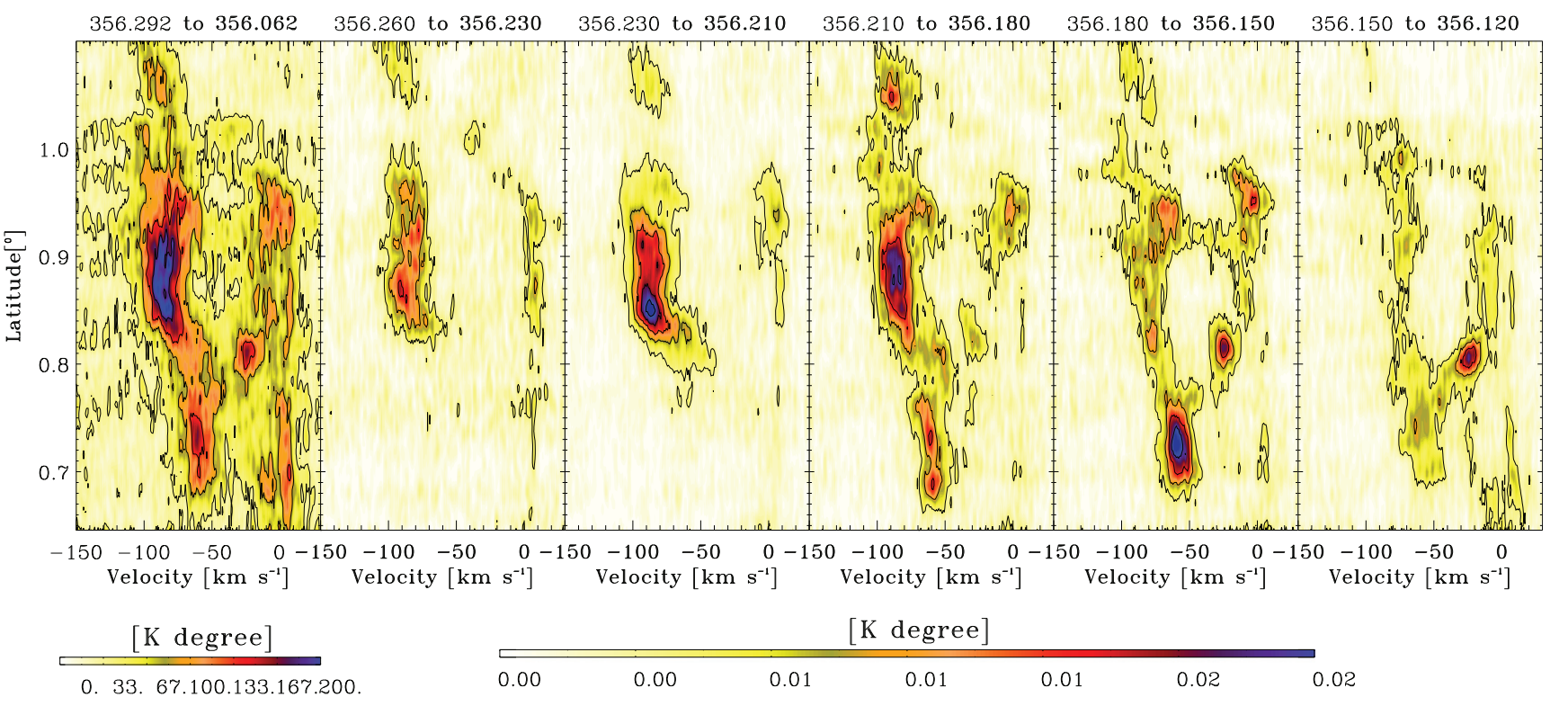

Fig. D.11. Latitude-velocity maps of the CS emission integrated in the complete longitude range from $356^{\circ} .29165$ to $356^{\circ} .06249$ (left panel), and in longitude steps of $108^{\prime \prime}$ (subsequent panels). 
SO

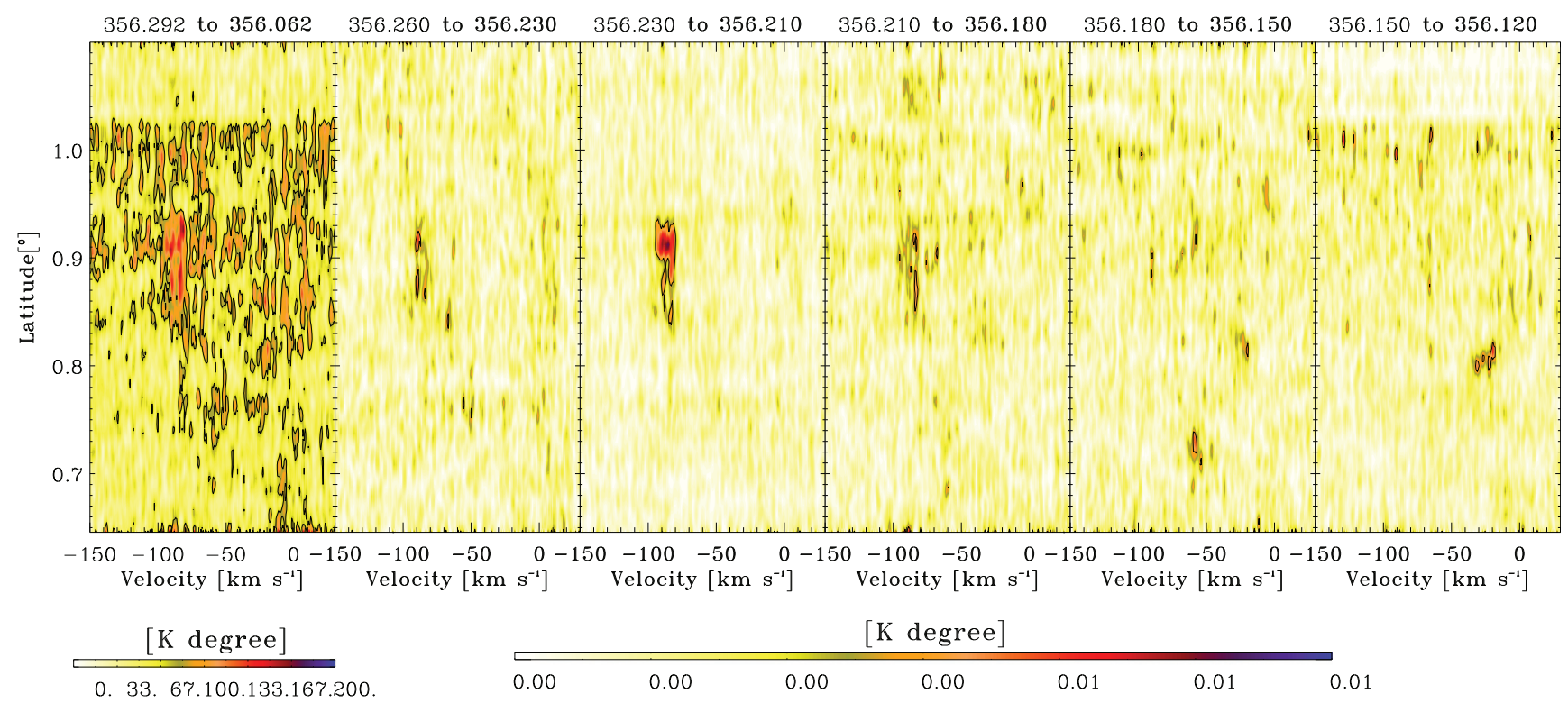

Fig. D.12. Latitude-velocity maps of the SO emission integrated in the complete longitude range from $356^{\circ} .29165$ to $356^{\circ} .06249$ (left panel), and in longitude steps of 108" (subsequent panels).

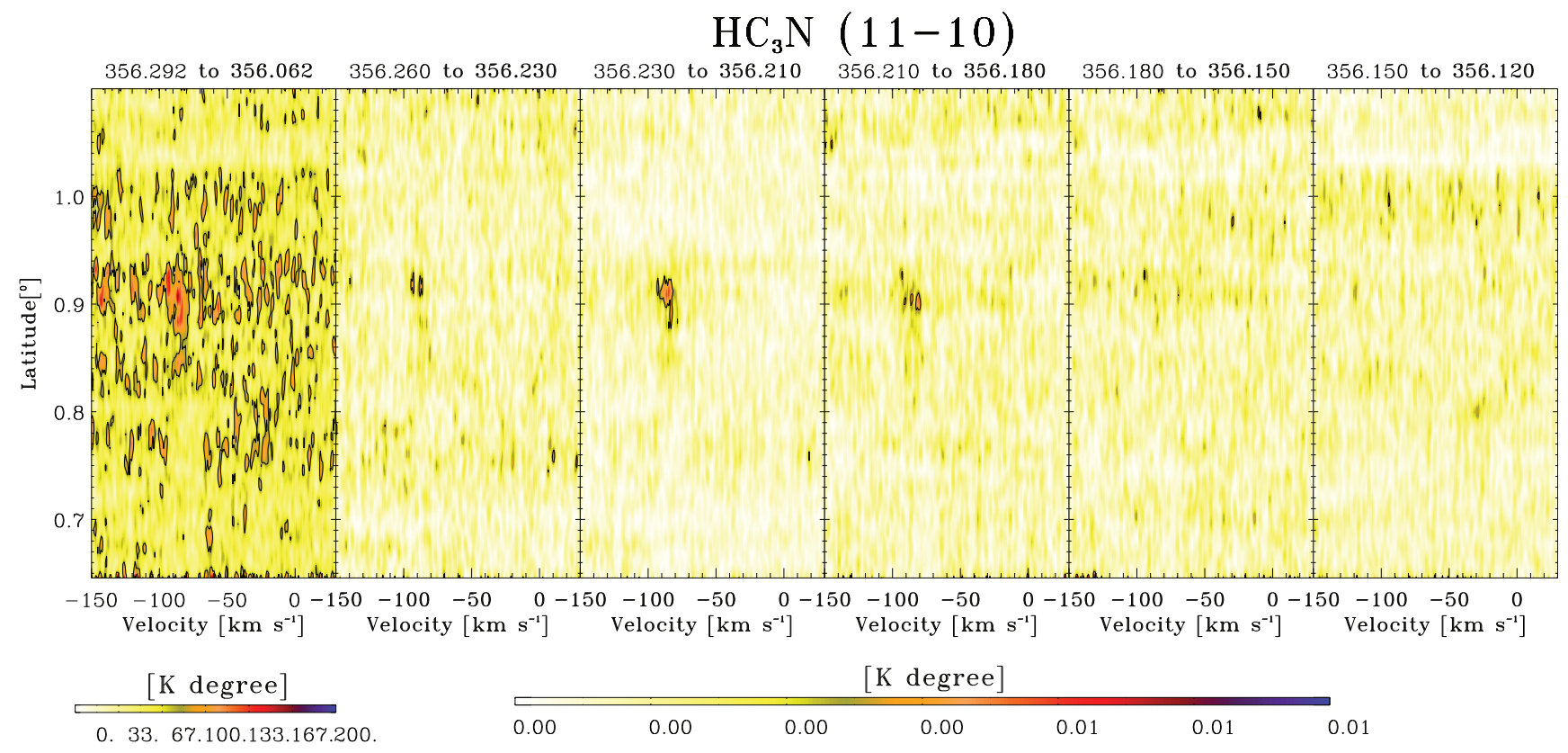

Fig. D.13. Latitude-velocity maps of the $\mathrm{HC}_{3} \mathrm{~N}(11-10)$ emission integrated in the complete longitude range from $356^{\circ} .29165$ to $356^{\circ} .06249$ (left panel), and in longitude steps of 108" (subsequent panels). 